State of Alaska

DEPARTMENT OF NATURAL RESOURCES

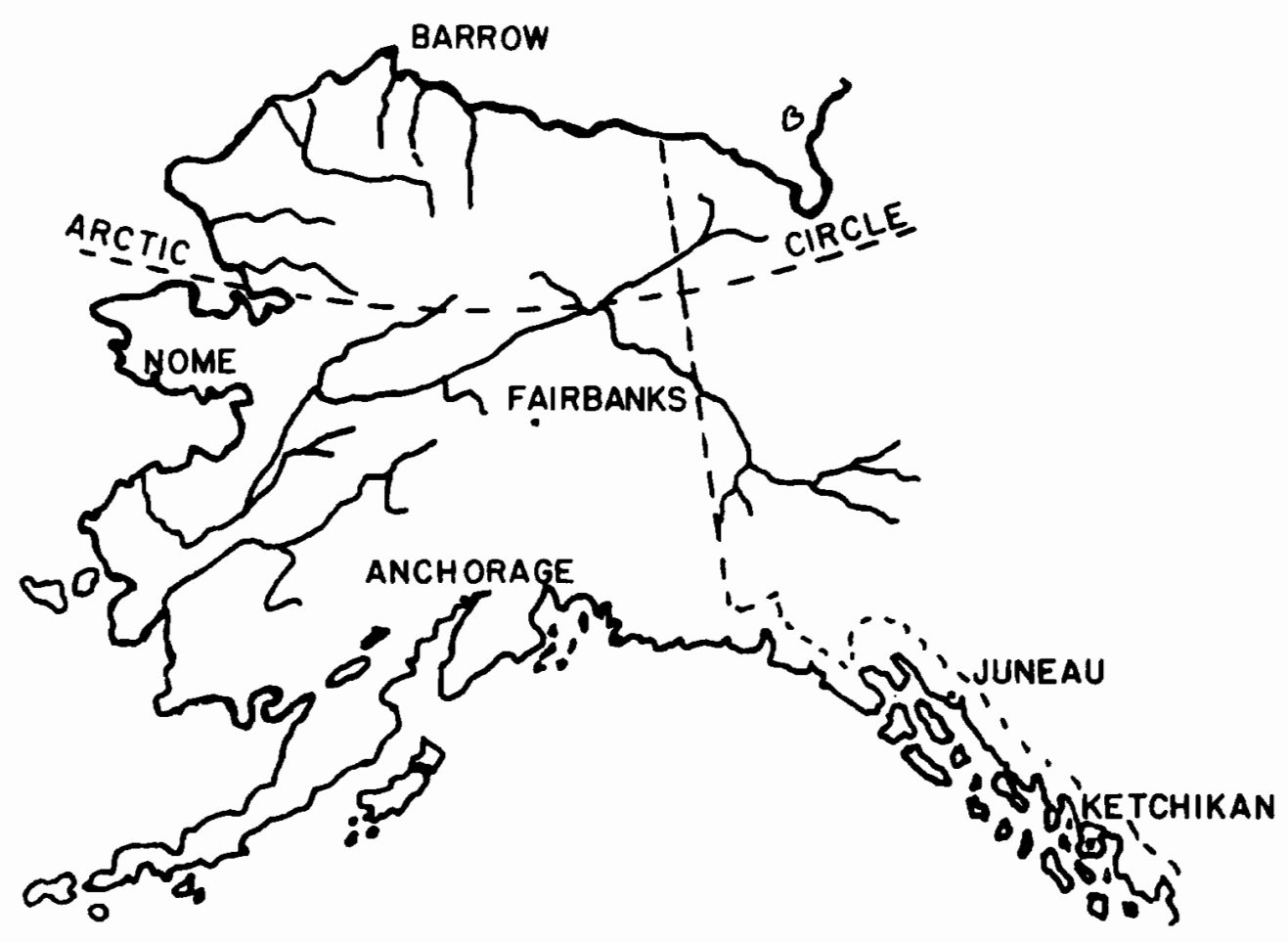

DIVISION OF MINES AND MINERALS

REPORT

FOR THE YEAR 1967
Property of DOGS LIBRARY

COLLEGE, ALASKA 


\author{
STATE OF ALASKA \\ Walter J. Hickel - Governor \\ Department of Natural Resources \\ Thomas E. Kelly - Commissioner \\ DIVISION OF MINES AND MINERALS \\ James A. Williams - Director
}

REPORT

FOR THE YEAR

1967

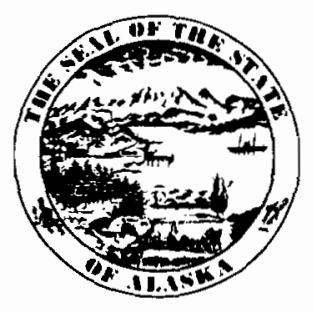

College, Alaska 
UIVISION OF IIINES AIND MIINERALS STAFF

December 31, 1967

Headquarters Office, Maintenance Building, Box 5-300, College

James A. Williams, Director

Gordon Herreid, Mining Geologist

Gilbert R. Eakins, Mining Geologist

Roderick R. Asher, llining Engineer

Theodore Vance, Mining Engineer

Paut L. Anderson, Laboratory Supervisor

Ivamok Cho, Assayer-Chemist

Donald R. Stein, Assayer

Michael Mitchell, Jr., Minerals Lab. Technician

Mary E. Shrewsbury, Administrative Assistant

Eleanor L. Pierce, Mining Information Specialist

Mary L. Hartley, Secretary

Patricia E. Burgess, Clerk Typist

Anchorage Uistrict Office, 3001 Porcupine Drive

Thomas R. Marshall, Jr., Petroleum Supervisor

Karl L. VonderAhe, Petroleum Engineer

0. K. Gilbreth, Jr., Petroleum Engineer

Harry W. Kugler, Petroleum Geologist

Robert E. Larson, Petroleum Engineer

Dorothy L. Chick, Mining Information Specialist

Martha A. Young, Clerk Stenographer

Joan iv. Agen, Clerk Stenographer

Juneau Uistrict office, Room 509 Goldstein Bldg... Pouch M.

Mildred E. Zenger, Administrative Assistant \& Mining Information Snecialist 
Honorable Thomas E. Kelly, Commissioner Department of Natural Resources

Pouch M

Juneau, Alaska 99801

Dear Sir:

It is a pleasure to transmit to you this Annual Report of the Division of Mines and Minerals covering the calendar year 1967. Summaries of mineral and petroleum production, exploration, and developments during the ycar are outlined. The activities and accomplishments of the Division are outlined, our geological investigations and petroleum regulatory work are briefly described, and details on information and services available to the public are included.

Each year continues to be more significant than the previous one for the Alaska petroleum industry. Exploratory drilling resulted in one oil well and three gas wells. Levelopment drilling resulted in 38 oil wells plus 23 still drilling at the end of the year. There are now seven oil fields and 18 gas fields in the State. Eleven platforms were operational in Cook Inlet with three more planned. Twenty-six miles of dual underwater pipeline were laid. More than $\$ 262$ million was spent by the industry in Alaskan exploration, development, and construction, exclusive of marketing and sales activities. Oil and gas production increased to $\$ 95$ million for the year.

Though not spectacular, mining exploration work by major companies is increasing, and interest in Alaskan mining possibilities continues to grow.

Direct revenue to the State from minerals totaled more than $\$ 35$ million in 1967. Better years lie immediately ahead.

This Division will continue to foster and assist the growth of the minerals industries in the best interest of the State.

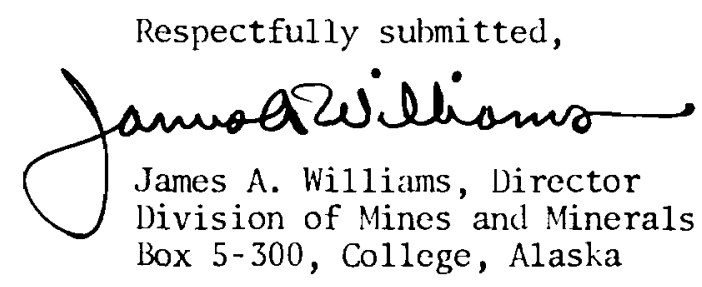


Title Page

Division of Mines and Minerals Staff

Letter of Transmittal

Table of Contents

THE MINING INDUSTRY

Mineral Production $\quad 7$

$\begin{array}{ll}\text { Precious Metals } & 10\end{array}$

Base Metals 10

Nonmetalics 10

$\begin{array}{ll}\text { Coal } & 10\end{array}$

Prospecting and Exploration 11

$\begin{array}{ll}\text { Prospecting Costs } & 15\end{array}$

THE PETROLEUM INDUSTRY

$0 i 1$ and Gas Exploration $\quad 17$

Development Drilling and Production Activity 17

Production Summary 18

$\begin{array}{ll}\text { Benefits to the State } & 18\end{array}$

ABSTRACTS OF REPORTS

Farewe11 Progress Report

0il Fields of Cook Inlet, Alaska

New Petroleum Prospects, Shallow and Deep Bering Sea 56

Placer Prospects in North Bering Sea $\quad 56$

Progress Report on the Geology and Geochemistry of the Sinuk Area,
Seward Peninsula, Alaska

Geochemical Report of the Wood River Lakes-Tikchik Lakes Region 57

A Geochemical Investigation of the Southwest Portion of the
40 Mile District, East Central Alaska

DIVISION OF MINES AND MINERALS

Genera 1

Organization, Functions, and Costs

Summary of Work Accomplished in 1967

Petroleum Branch Activities

Summary of $0 i 1$ and Gas Conservation Orders Issued in 1967

Reports Published During 1967

Reports to be Published Soon

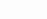

Division Programs for 1968

Prospector Assistance Program

ACTIVE PETROLEUM COMPANIES $\quad 76$

ACTIVE COAL MINES, $1967 \quad 78$

LIST OF ALASKA MINING \& EXPLORATION OPERATIONS ACTIVE DURING 1967

LIST OF REPORTS ISSUED BY THE DIVISION OF MINES AND MINERALS AND PRECEDING AGENCIES 
CONTENTS (Con't)

Page

Table I Mineral Production in Alaska 7

Table II Production of Major Commodities since 1949

Table III Physical Volume of Alaska Mineral Production 8

Figure 1 Annual Value of All Mineral Production 9

Figure 2 Barometer of Exploration Activity 16

Table IV State Total Production by Years 19

Figure $3 \quad$ Total $0 i 1$ Production Per Day 20

Figure $4 \quad$ Cook Inlet Area - Proven Fields and Exploratory Wells 21

Table V Summary of Petroleum Industry Statistics 22

Table VI Exploratory Wells Active in 1967

Table VII Wells Active in 1967

Table VIII Middle Ground Shoal Field - Cook Inlet Alaska 27

Table IX Middle Ground Shoal Field - Individual Well 0 il Production 28

Figure $5 \quad$ Middle Ground Shoal Field - 0 il Production 29

Table $X \quad 0 i 1$ Production by Months - Middle Ground Shoal and
Granite Point Fields

Table XI Granite Point Field 31

Figure $6 \quad$ Granite Point Field - $0 i 1$ Production 32

Table XII Granite Point Field - Individual Well 0il Production 33

Table XIII Swanson River Field

Figure $7 \quad$ Swanson River Field 0il Production 35

Table XIV Swanson River Field - 0il Production by Months 36

Table XV Swanson River Field, SRU, Individual Well Production 37

Table XVI Swanson River Field, SCU, Individual Well Production 38

Table XVII Swanson River Field, SCU, Injection Project 39

Table XVIII Trading Bay Field 41

Figure $8 \quad$ Trading Bay Field - 011 Production 42

Table XIX Trading Bay Field - Individual Well Production 43

Table XX Trading Bay and McArthur River Fields - Production by Months 43 
CONTENTS (Con' $t$ )

$\begin{array}{lll}\text { Table XXI } & \text { McArthur River Field } & 44 \\ \text { Table XXII } & \text { McArthur River Field - Individual We11 0i1 Production } & 44 \\ \text { Figure } 9 & \text { McArthur River Field 0il Production } & 45 \\ \text { Table XXIII } & \text { Gas Production } & 46 \\ \text { Table XXIV } & \text { South Barrow Gas Field } & 48 \\ \text { Table XXV } & \text { Kenai Gas Field } & 49 \\ \text { Figure 10 } & \text { Kenai Gas Field - Gas Production } & 50 \\ \text { Table XXVI } & \text { Kenai Deep Gas Field } & 51 \\ \text { Table XXVII } & \text { Sterling Gas Field } & 52 \\ \text { Figure 11 } & \text { Sterling Gas Field Production } & 53 \\ \text { Figure 12 } & \text { Central and Northern Alaska Well Locations } & 54 \\ \text { Table XXVIII } & \text { Well Records Released During 1967 } & 64 \\ \text { Table XXIX } & \text { Well Records to be Released During 1968 } & 65 \\ \text { Table XXX } & \text { Sample and Core Inventory } & 66 \\ \text { Table XXXI } & \text { Prospector Assistance Program - 1967 } & 75\end{array}$




\section{THE MINING INDUSTRY}

Mineral Production

Tabie I presents a comparison of mineral production during 1966 and 1967. During 1967 total mineral production was estimated to be $\$ 737.1$ million compared to $\$ 86.3$ million in 1966 . Sand and gravel production increased from $\$ 21.8 \mathrm{million}$ to $\$ 27.7 \mathrm{million}$. $0 \mathrm{il}$ and gas production increased from $\$ 50.3 \mathrm{milli}$ ion to $\$ 95.4$ million with the cook Inlet area being the predominant producer. Coal production increased slightly over 1966. The cumulative total mineral production of Alaska is now $\$ 2,418,500$ of which $\$ 759,100,000$ has been in gold.

Direct revenue to the State from mineral production and exploration, which includes royalties, filing fees, lease rentals, production taxes, and sale of sand and gravel, amounted to $\$ 35,630,000$ in 1967 . This does not include individual income taxes and business license taxes from the mineral industry and service groups.

Table I - Mineral Production in Alaska

1966

$1967(1)$

$\begin{array}{crrr}\text { Quantity } & \begin{array}{c}\text { Value } \\ \text { (Thousands) }\end{array} & \text { Quantity } & \begin{array}{c}\text { Value } \\ \text { (Thousands) }\end{array} \\ 8 & & W & W \\ 927 & \$ 6,953 & 930 & \$ 7,178 \\ 27,325 & 956 & 26,000 & 910 \\ 14 & 4 & W & W \\ W & W & 161 & 79 \\ 33,646(2) & 6,335 & 39,927(2) & 7,268 \\ 14,376(3) & 44,083 & 28,917(3) & 88,187 \\ 17,457 & 21,793 & 22,426 & 27,683 \\ 7 & 9 & 8 & 12 \\ & 6,167 & & 5,830 \\ & \$ 86,300 & & \$ 137,147\end{array}$

Antimony---Short tons antimony content Coal-_-_-_-_----thousand short tons

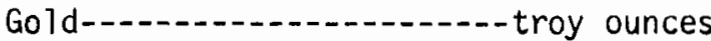
Lead----short tons recoverable content Mercury--.----------76-pound flasks Natural Gas--.-----milition cubic feet Petroleum, crude-..-.-thous and barrels Sand and Grave1-..-thous and short tons Silver--.---.--thousand troy ounces Undistributed (4)

Totals

(1) Figures for 1967 except petroleum and natural gas are preliminary and subject to revision.

(2) Includes only gas sold.

(3) Includes only oil sold. Additional small amounts were produced during testing of new wells.

(4) Undistributed includes barite, copper, gem stones, lead, mercury, peat, platinum group metals, stone, tin, and uranium.

W Withheld and included under "Undistributed" to avoid disclosing individual company confidential data.

Note: Above statistics prepared under a cooperative agreement for the collection of mineral data between the bureau of Mines, United States Department of the Interior, and the Division of Mines and Minerals, Department of Natural Resources, State of Alaska. Figures for coal, petroleum, natural gas, and undistributed commodities are presented on authority of the Division of Mines and Minerals only. 
Table 11 - Production of Major Commodities since 1949 Dollar Value (Thousands)

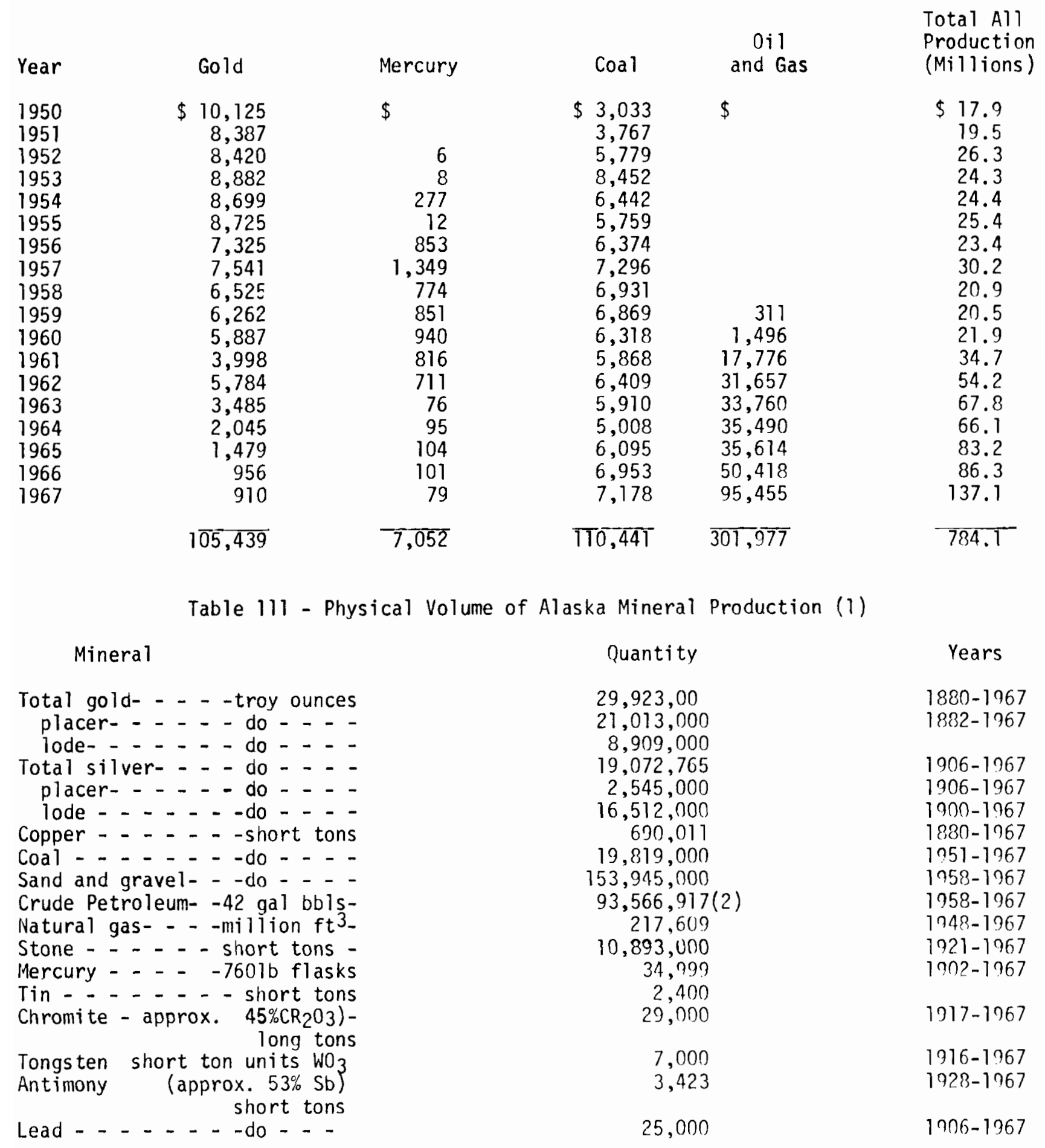

(1) Except platinum, uranium, barite, and other commodity fiqures which are confidential. 1967 production estimated and included in total.

(2) Only other crude petroleum recorded production was from the Katalla area. From 1301 to $1932,154,000$ barrels of oil were produced there. 
Figure 1 - Annual Value of All Mineral Production - Alaska 1900 - 1767

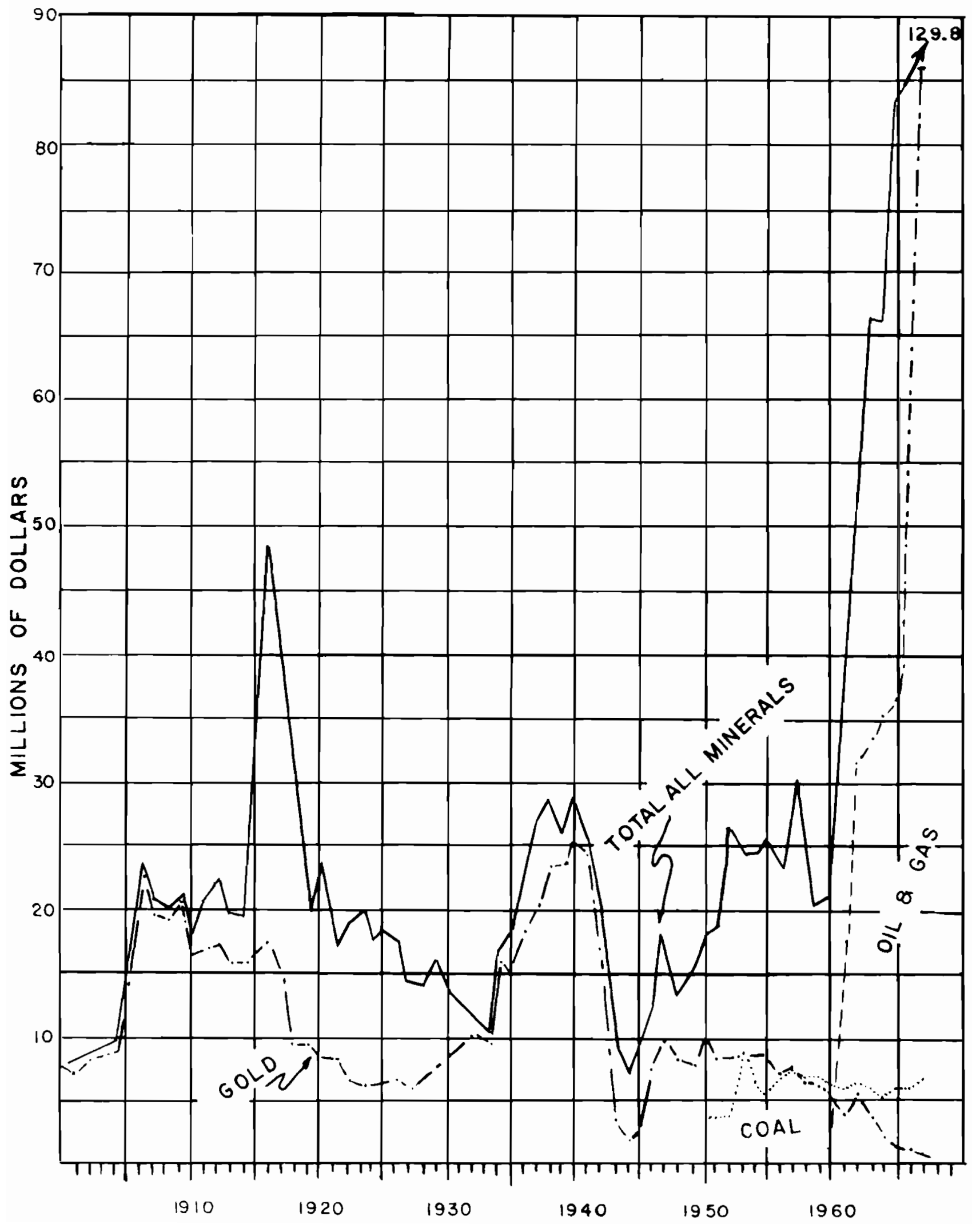




\section{Precious Metals}

Gold production continued to decline and will decline further unless some incentive such as an increase in price or direct subsidy occurs.

The United States Smelting Refining and Mining Company announced a suspension of dredging operations at the close of the placer season on Chicken Creek. A dredge at Hogatza River will continue.

The Goodnews Bay Mining Co. is still the only major producer of platinum in the United States. Increasing demand and uses for platinum have resulted in very high prices for the meta1. This has stimulated further exploration of placer deposits in the Saimon River area which were previously uneconomic to mine, mostly because of depth. Interest in possible nearby lode deposits has continued.

Renewed interest in silver has taken place throughout the State in the wake of soaring prices for the metal. The Division of Mines has received numerous inquiries about old silverlead mines and deposits that were not previously considered important.

\section{Base Metals}

During 1967, mercury production declined slightly from the previous year. Prices of mercury have been quite good and interest in new deposits is very active, but mining remained limited to small-scale operations. A large low-grade deposit in the sleetmute area was being explored.

No significant copper mining was in progress during the year. Kennecott Copper Corporation's property at Bornite, in the Kobuk area, is still in the development stage.

\section{Nonmetallics}

Sand and gravel increased in volume over the previous year. Principal producers were the State Department of Highways, Army Corps of Engineers, and the State Division of Lands.

Small quantities of peat and jade were produced for local markets.

The Kendrick Bay uranium mine on Prince of Wales Island did not operate during the year but plans to start production in the near future.

\section{Coal}

The Nenana coal field operators, Usibelli, Inc. and Vitro Minerals Corp., continued to supply the northern military bases. Usibelli also shipped 105,000 tons to the Anchorage area. Evan Jones Coal Co. delivered 210,000 tons to the Anchorage military bases from its mine in the Matanuska field.

The U. S. Bureau of Mines reports that Usibelli received $\$ 5.25$ per ton, Vitro $\$ 5.65$, and Evan Jones $\$ 12.88$ per ton for coal contracted to the military.

A threat to Matanuska coal production looms in the future in the form of conversion from coal to natural gas by Anchorage area military bases. Congress has approved $\$ 1,980,000$ for the conversion.

Interest in coking coal for export remains high. A feasibility study and exploration of the Bering River coking coal deposits near Cordova was started by Cortella coal Corp. of Cordova for Sumitomo Co., one of three Japanese firms interested in the deposits. One of the primary concerns of the study is the method of transporting coal from the mine to Cordova, the nearest deepwater port. The possibility of moving coal by pipeline under water pressure is being investigated. 
Prospecting and Exploration

Northwestern

The largest operation in this area continues to be Kennecott Copper Corporation and its exploration subsidiary Bear Creek Mining Company.

Kennecott's 1070 foot shaft, which was flooded, has been plugged. Eight carloads of cement were flown to the property and the shaft was filled to a depth of 23 feet from the bottom. The water has been pumped down to the plug, which seems to be holding, and underground exploration work is again proceeding with drifting and drilling at the 700 and 1000 foot levels.

Bear Creek Mining Company has continued intensive exploration in the general Kobuk area where the Bornite operation is located. It also took over work at the Hannum Creek lead-silver prospect on the Seward Peninsula where Bunker Hill had drilled the previous summer.

Interest in offshore prospecting, especially off the Nome beaches, continued at a high pitch. More than 1,000 offshore prospecting permits have been issued by trie State within the three-mile limit. Most of these surround the Seward Peninsula. A few offshore production leases have been issued, all in the Nome area, but no significant production has yet resulted. Shell 0il Company has done considerable seismic and bottom sampling work. It pioneered experimental work with a sonic sampling drill operating on the same principle as the successful high-frequency sonic pile driver. Martin Dredging Company converted a surplus Navy mine sweeper into a suction dredge and made a serious attempt to start production off Daniels Creek, long known as a "hot spot". Operational problems developed and modification of the digging and gold saving equipment is necessary. Some work has been done out toward the western part of Seward Peninsula to determine the amount of placer tin that may lie offshore from the known placer tin areas.

The U. S. Geological Survey and U. S. Bureau of Mines spent considerable effort this year in the heavy metals program. The USGS brought at least two mobile labs to Alaska in support of its Field parties. The USBM's chief effort was the cruise of the vessel VIRGINIA CITY to the waters offshore from Nome where it did experimental drilling and other bottom sampling. Results have been described as very promising. The USGS cooperated in this work. The two agencies were also engaged in other exploration and research in attempts to find new gold resources.

Newmont Exploration, Ltd. and United States Refining and Mining Company had a sizeable crew supported by helicopter doing reconnaissance work on Seward Peninsula and along the main Alaska Range.

\section{Central}

The United States Smelting Refining and Mining Company operated two dredges during the year. The dredge at Chicken worked during the season and was then shelved permanently as the dredging deposits in this area have become submarginal under present economic conditions. The Hogatza dredge operated through the season and will continue for a few more seasons. The company also conducted exploration throughout Alaska.

Exploration at the Busty Belle property in the Fairbanks district was continued during the year. Approximately $530^{\prime}$ of drifting and cross-cutting was accomplished, and 300 feet of core drilling performed. The owners recently announced plans to construct a 50-ton mill which will also be available for custom milling ores from other properties.

A property on Steamboat Creek has been drilled recently by Pacific Construction Company, and work will be resumed in the spring.

Harold Hassel and crew did stripping and exploration on his Ready Bullion Creek placer property in the Fairbanks area. 
Activity at the Keystone Mines, Fairbanks area, consisted of general exploration and excavation on outcrops. area.

Stripping for outcrops was performed at the Bertholomae lode property in the Fairbanks

Hanna Mining Co. explored by sluicing and other means the Ignaty mercury prospect in the Kuskokwim mercury belt and investigated the Buzby prospect in the Bonnifield district.

Diamond Alkali of Cleveland, Ohio had a crew of nine men drilling the mercury property in the Kuskokwim district formerly held by Russel Schaefer.

Tennessee Corp. continued drilling the Pass Creek copper show north of the Denali Highway and supported a reconnaissance prospecting crew in various areas as in former years.

Hecla Mining Company investigated possibilities in the White River and Nabesna areas.

Southcentral

Wrangell Consolidated Mining Company of Chitina had a crew of 12 men concentrating and shipping copper ore from the old Kennecott property. The concentrates were flown to Chitina, trucked to Anchorage and then from Anchorage over the Alaska highway to Vancouver where they were loaded on freighters bound for Japan.

Slim Blood of Valdez did some exploration at the old Green Butte copper mine and Gordon Burdick did likewise at the Nicolai property.

The New Concept Mining Company of Florida had a crew working at Martin Radovan's Binocular Prospect on Glacier Creek.

George Gilbertson of Fairbanks was reported to be placering on Dan Creek, which, like the above mentioned properties, is in the Nizina, or McCarthy District.

State Highway Department plans call for completion of the bridge at Chitina and the road into the Nizina District within five years. This will accelerate exploration in that district.

Conwest Exploration Company Ltd. of Vancouver was reported investigating the Tuxedni iron deposit on the west side of Cook Inlet.

Southwestern

St. Eugene Mining Company (Falconbridge) continued its studies of the Kasna Creek copper property in the Iliamna region with a crew of nine men doing geological and geophysical work. This property is looking good but transportation is the chief problem. At present it is rather inaccessible.

Several companies and consultants showed a great deal of interest in the sulphur deposits of the Aleutian Islands.

\section{Southeastern}

Dynasty Exploration of Vancouver has been working with at least eight men on a group of 300 claims on the southern end of Prince of Wales Island where the possibility of porphyry copper exists. This was a discovery of Canadian prospector James Walper. Dynasty has done trenching and geophysical work and is now reportedly preparing to drill.

In an issue of our monthly Mines and Petroleum Bulletin we reported that the U. S. Park Service had purchased the Alaska Chief copper mining property within Glacier Bay National Monument. It develops that our identification of the purchaser of this mining property may be an error, but no denial was ever received from the Park Service. It now appears that it was a private conservation group that purchased the property. 
Global Marine, Inc. prospected in Gastineau Channel between Juneau and Douglas Island. The exploration program, which will continue into 1968, has been confined to reconnaissance sampling and marine seismic work to determine possible value and thickness of sediments in the Channel. The company has also been active offshore at Nome.

General

Homestake Exploration of Vancouver has been busily looking at prospects throughout the State.

Hanna Mining Company has also been intensively investigating various prospects and hired a prospector to do reconnaissance geochem work in various areas.

Wallace MacGregor of Salt Lake City with a crew of geologists actively investigated a number of properties throughout the State until late in the season.

In the Rampart Report, published last summer by the Department of Interior, Secretary IJdall announced that the huge Rampart Dam project was not practical, but offered alternatives. One of these was a mineral development plan to increase the work of the USGS and the USBM in the north and to establish an Arctic Mineral Resources Institute in a building to be constructed on the University of Alaska campus. The Institute would be staffed by USGS and USBM scientists but would also include the State Division of Mines and Minerals and the University of Alaska College of Earth Sciences and Mineral Industry. Since the Division is now located at the University it is in a better position to participate in this Federal program as it develops.

The U. S. Geological Survey was active during the year in a number of activities. The current list of activities or projects include geologic mapping, mineral district mapping, geochemical sampling and mapping; reconnaissance and detailed geophysical surveys; engineering geology studies of earthquake effects; and paleontologic and mineralogic work to support other project activities. Specific topics investigated include the identification of heavy metals resources of the State; petroleum, including oil shales and coal resources; marine geology, particularly of the Bering Sea region; study of the earth's thermal regime; distribution and history of surficial deposits and other special studies. deposits.

The U. S. Bureau of Mines continued its program of investigating coal and other mineral

The following USGS and USBM publications were released in 1967. Open file reports are usually available for study at the various USGS, USBM and Division of Mines and Minerals Offices in Alaska. Copies of open file reports by reproduction can usually be obtained at private expense. USGS numbered bulletins and professional papers may be purchased from the U. S. Government printing office. U. S. B. M. information circulars and reports of investigations may be purchased from the Publication Distribution Office, U. S. Bureau of Mines, Pittsburgh, Pa. Division publications are listed elsewhere in this report.

USGS Open File Report, Preliminary Geologic Map of Kodiak Island and Vicinity, Alaska, by George W. Moore.

USGS Open File Report, Surficial Deposits of the Iliamna quadrangle, Alaska, by Robert L. Detterman and Bruce L. Reed.

USGS Open File Report, Metallic Mineral Resources Map of the Fairbanks quadrangle, Alaska, by Edward H. Cobb.

USGS Open File Report, Four Preliminary Gravity Maps of Parts of Alaska, by David F. Barnes.

USGS Open File Report, Copper Analyses of Selected Samples, Southwestern Brooks Range, Alaska, by W. P. Brosge, H. N. Reiser, and I. L. Tailleur. 
USGS Open File Report, Location and Description of Lode Prospects in the Livengood Area, East-Central Alaska, by Robert L. Porter and Robert M. Chapman.

USGS Open File Report, Results of Stream Sediment Sampling and Bedrock Analyses in the Eastern Part of the Iliamna Quadrangle and at Kasna Creek, Lake Clark Quadrangle, Alaska, by Bruce L. Reed.

USGS Open File Report, Profiles Showing Configuration and Probable Bottom Deposits as Interpreted from Fathometer Traverses Across and Along Parts of Gastineau Channel, near Juneau, Alaska, by Robert D. Miller.

USGS Metallic mineral resources map of the Charley River Quadrangle, Alaska, compiled by Edward H. Cobb.

USGS Metallic mineral resources map of the Circle Quadrangle, Alaska, compiled by Edward H. Cobb.

USGS Metallic mineral resources map of the Eagle Quadrangle, Alaska, compiled by Edward H. Cobb.

USGS Metallic mineal resources map of the Livengood Quadrangle, Alaska, compiled by Edward H. Cobb.

USGS Map 1-492, Regional Geological Map of the Candle Quadrangle, Alaska by William W. Patton.

USBM Open File Report, Resume of Information on Alaskan Bituminous Coals with Particular Emphasis on Coking Characteracteristics by Robert W. Warfield.

USBM Open File Report, Sampling the Moth Bay Zinc-Copper Deposit, Revillagigedo Island, Southeastern Alaska, by R. W. Warfield and R. R. Wells.

USBM Information Circular 8331, Production Potential of Known Gold Deposits in the United States, by Bureau of Mines field staff.

USGS Professional Paper 512, Geology of the Iniskin-Tuxedni Region, Alaska, by Detterman and Harsock.

USGS Professional Paper 567, Dictionary of Alaska Place Names by Donald J. Orth.

USGS Professional Paper 550-D contains many research articles of which the following pertain to Alaska: Cretaceous Stratigraphy of the Kamishak Hills, Preliminary report on a plutonic belt in West-central Alaska, and Potassium-argon ages of Tertiary plutons in the Prince William Sound region, Alaska. 
Prospecting Costs

The following costs are based on a five-year average of expenditures by participants in the State Prospector Assistance Program. Expenses have averaged $\$ 22.81$ per man day. In the five year period $\$ 96,357.66$ was spent covering 4,225 man days. The total cost to the State over the five years was $\$ 67,760.15$ or about 70 percent of the total.

The cost distribution for 1966 and 1967 is as follows:

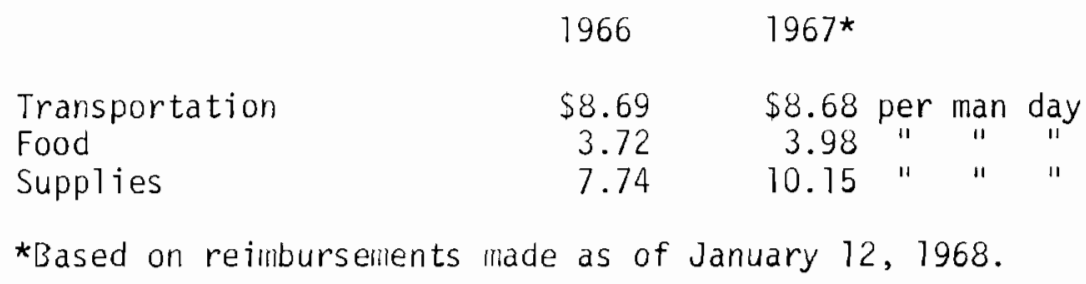

Transportation includes aircraft, tracked vehicles, rubber tired equipment, and boats used to travel to and from the prospecting area and to transport supplies and equipment. Food includes groceries and other staple, consumable items. Supplies include tools, camping equipment, prospecting equipment, fuel, and general supplies. The increase in the cost of supplies reflects greater use of heavy equipment in prospecting, especially in exploring claims staked under the program in previous years.

Travel by charter aircraft is most commonly used. Small fixed-wing airplanes are available at most towns in Alaska. Charter rates vary froml $\$ 20-40$ per hour for a Piper Cruiser to $\$ 40-60$ per hour for a Cessna 180. Helicopters are available in Ketchikan, Juneau, Fairbanks, and Anchorage. Charter rates vary from $\$ 100-135$ per hour with a three-hour guaranteed minimum flying time. 
Figure 2 - Barometer of Exploration Activity - claims staked
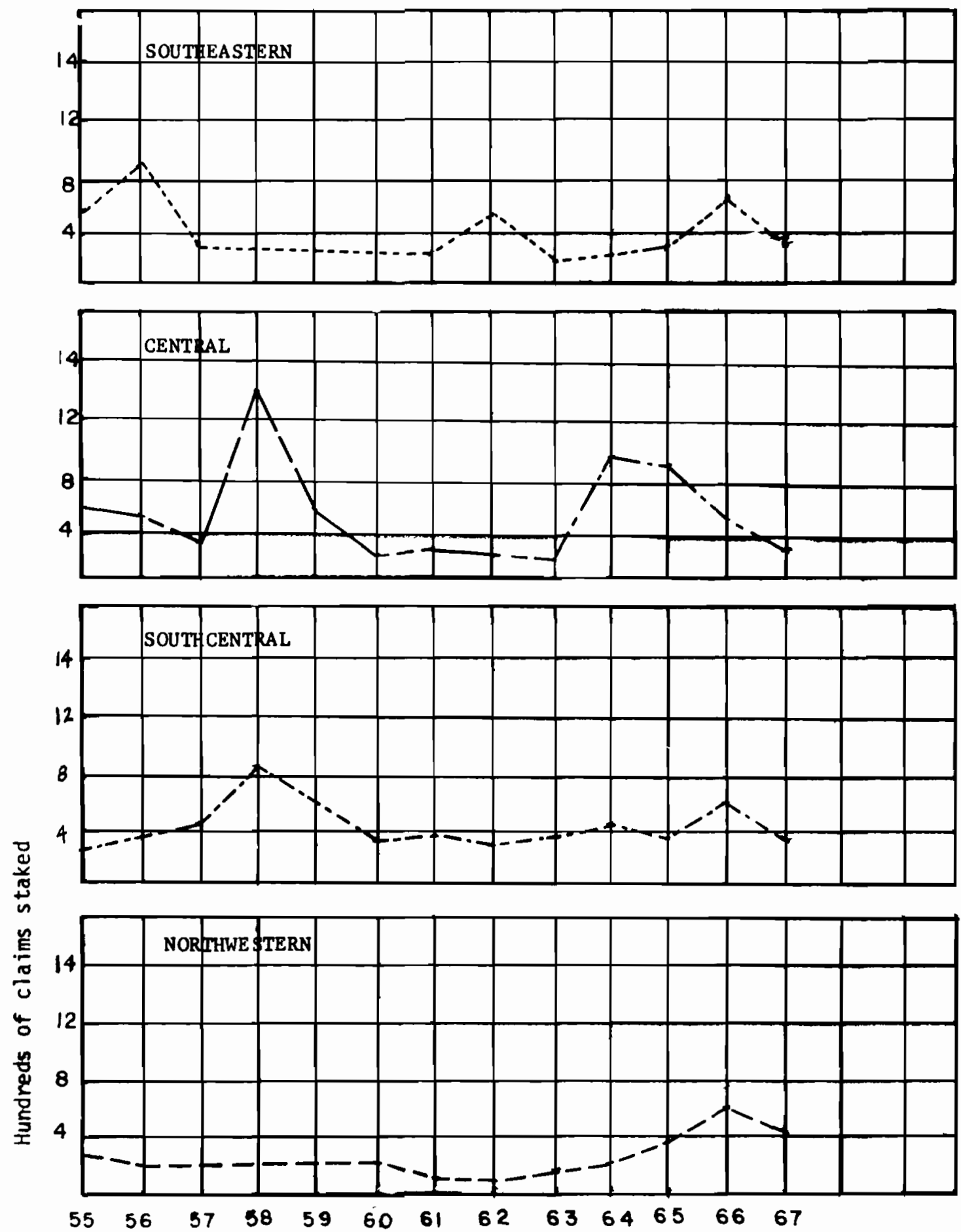


\section{THE PETROLEUM INDUSTRY}

\section{$0 i 1$ and Gas Exploration}

(See Table VI for specific details on wells and the list in the back of this volume for active companies)

Thirty-three exploratory wells were active in three geologic provinces during 1967. The results were one oil well, three gas wells, three suspended wells, and twenty-three dry holes with three wells still active at the end of the year. Eighteen wells were onshore ventures while the remaining fifteen were drilled offshore in Cook Inlet. This compares with forty-two exploratory wells active in 1966.

The Atlantic Richfield Company accounted for the oil discovery with the \#1 Trading Bay State, located about 1 1/2 miles northeast of the Trading Bay Field. Texaco Incorporated and the Atlantic Richfield Company will each install a platform to develop the area northeast of the Trading Bay Field.

The Texaco \#3 Nicolai Creek Unit gas well extended the Nicolai Creek Field about $11 / 2$ miles to the north. The \#1-A Beaver Creek gas well was drilled by Marathon 0il Company as a relief well for the \#l Beaver Creek, which blew out during drilling operations and was subsequently plugged and abandoned. The last of the three gas discoveries is the Union 0il Company of California \#l Kenai Deep Unit which discovered deeper productive sands within the producing limits of the Kenai Gas Field. These oil and gas discoveries were all in the Cook Inlet basin.

The North Arctic Slope had about the same activity as in 1966. The Atlantic Richfield Company abandoned the Susie Unit \#l but was still drilling ahead on the Prudhoe Bay \#1. The Union $0 i 1$ Company of California abandoned the Kookpuk \#l.

The Bristol Bay basin was tested by one we11, the Cities Service 0i1 Company \#1 Painter creek, subsequently abandoned.

Geophysical activity was about the same as in 1966. The Gulf of Alaska, Bristol Bay, Cook Inlet and Kenai Peninsula accounted for most of the activity. Most of the geologic field parties were on the Alaska Peninsula.

\section{Development Drilling and Production Activity}

Development drilling in 1967 was centered mainly on the drilling platforms in Cook Inlet. Seventy-four development wells were active of which seventy-two were offshore. These operations resulted in completion of thirty-eight oil wells, two dry holes, no gas wells, eleven suspended wells, and twenty-three wells still active at the end of the year. Of the thirty-eight oil wells, sixteen were completed in the Granite Point Field, thirteen in the Middle Ground Shoal, three in the McArthur River, and six in the Trading Bay Field.

Construction was completed on five additional offshore drilling-production type platforms, making a total of eleven now operational in Cook Inlet. Nineteen rigs were drilling continuously from these in four different fields. Eight of the platforms can handle two rigs drilling simultaneously, while three have provisions for only a single rig. Three additional platforms are to be installed next year.

Over twenty-six miles of dual pipelines were installed in cook Inlet, most of which connects platforms in the McArthur River area to shore. The forty-two-mile twenty-inch common carrier line of Cook Inlet Pipeline Company on the west side of the Inlet was completed from Granite Point to the Drift River facility. An offshore loading dock with mooring dolphins capable of handling ships up to sixty-foot draft was placed into operation late in the year at the Drift River terminal. Slightly over one million barrels of storage has been provided. This facility ultimately will be able to handle the giant super-tankers. 
A11 oil production in Alaska is handled by automatic custody transfer equipment. Individual well test facilities have been installed on each platform to facilitate gatherings of individual well information. Production is transported to shore through twin underwater pipelines to central batteries where it is treated, stored, and sold.

High productive rates have been indicated by well completions this year. The highest productive well was slightly over 9,200 barrels of $0 i l$ per day, with most wells testing around 2,000-4,000 barrels. The average per-wel1 productivity in December was 1,376 barrels per day, which is the highest in the nation. Alaska now ranks about tenth in the nation in total oil production and about seventh in reserves.

There are now seven commercial oil fields and eighteen gas fields in the state including Naval Petroleum Reserve No. 4. Five oil fields and four gas fields are in production. A drilling and development type platform will be installed in the Ivan Bering, Trading Bay, and North Cook Inlet Fields next year with onstream production anticipated by late 1968 or early 1969. Production from the North Cook Inlet Field will be utilized for the liquid methane gas plant now being built near the city of Kenai. This facility will help establish a market for large quantities of gas production from the area.

The fourteen shut-in gas fields are awaiting development of a commercial market.

Production Summary

Total oil production for 1967 amounted to 28,917,464 barrels. The daily rate for the 1ast week of the year was 109,455 barreis as compared to a rate of 51,500 barrels per day at the beginning of the year. This represents an increase in daily production rate of 112 per cent. Total cumlative oil production was 93,566,917 barrels at the end of the year.

There are now 105 active producing oil wells including ten duals counted as twenty wells. In addition, there are thirty wells which have produced at commercial rates but have been shutin or suspended for various reasons. Three of these shut-in producers are in Naval Petroleum Reserve No. 4.

Total gas production for the year was $62,868,777$ MCF (thousand cubic feet), which includes 475,382 MCF produced from the South Barrow Field in NPR No. 4. Gas sales were 39,927,463 MCF. Gas used on leases amounted to 11,409,331 MCF. The remaining 11,531,983 MCF were accounted for as being line losses, flared, or blown.

There were twenty-three producing gas wells in five gas pools with twenty-five closed-in wells in thirteen pools wajting for market. Four wells in two other pools which previously have produced in paying quantities are suspended but not yet abandoned. The production from the individual pools is shown elsewhere in this report.

Benefits to the State

Income to the State of Alaska derived from $0 i 1$ and gas lease rentals and royalty payments on both state and federal lands amounted to $\$ 13,943,556$ for the year. Bonus payments for oil and gas leases were $\$ 20,256,124$. Production, disaster, and conservation taxes were estimated to be $\$ 1,430,000$, making a total direct $0 i 1$ and gas income of about $\$ 35,630,000$ in 1967 . In addition to this information, Table $V$ on the following pages gives detail statistical data of interest resulting from oil and gas operations in the State of Alaska. 


\author{
Table IV \\ State Total Production \\ Summary by Years
}

0 il-Bbls.

\begin{tabular}{|c|c|c|}
\hline Year & $0 i 1$ & Cumulative \\
\hline \multicolumn{3}{|c|}{ Prior to 1958} \\
\hline 1958 & 35,754 & 35,754 \\
\hline 1959 & 186 & 222,344 \\
\hline 196 & & 780,3 \\
\hline 1961 & 6,326 & $7,106,8$ \\
\hline 1962 & $10,259,110$ & $17,365,954$ \\
\hline 1963 & & 15,918 \\
\hline 1964 & 11,053 & $39,159,790$ \\
\hline 1965 & $11,128,545$ & $50,288,335$ \\
\hline 196 & $14,358,2$ & $64,646,548$ \\
\hline 196 & 20017 & $93,563,994$ \\
\hline
\end{tabular}

Gas - MCF 14,65 psi \& $60^{\circ} \mathrm{F}$

\begin{tabular}{rrrrr}
\hline Csg. Head & Cumulative & Gas Wel1* & Cumulative & \multicolumn{1}{r}{$\begin{array}{c}\text { Total } \\
\text { Cumulative }\end{array}$} \\
& & 829,832 & 829,832 & 829,832 \\
5,643 & 5,643 & 115,030 & 944,862 & 950,505 \\
27,291 & 32,934 & 132,624 & $1,077,486$ & $1,110,420$ \\
99,176 & 132,110 & 211,225 & $1,288,711$ & $1,420,821$ \\
$1,293,258$ & $1,425,368$ & 387,155 & $1,675,866$ & $3,101,234$ \\
$1,914,054$ & $3,339,422$ & $1,839,229$ & $3,515,095$ & $6,854,517$ \\
$2,808,011$ & $6,147,433$ & $8,213,056$ & $11,728,151$ & $17,875,584$ \\
$3,233,232$ & $9,380,665$ & $8,880,522$ & $20,608,673$ & $29,989,338$ \\
$3,842,367$ & $13,223,032$ & $8,701,080$ & $29,309,753$ & $42,532,785$ \\
$6,822,476$ & $20,045,508$ & $34,833,665$ & $64,143,418$ & $84,188,926$ \\
$22,539,948$ & $42,585,456$ & $40,360,557$ & $104,503,975$ & $147,089,431$
\end{tabular}

* Includes gas from South Barrow Field - NPR4 
TOTAL OIL PRODUCTION PER DAY

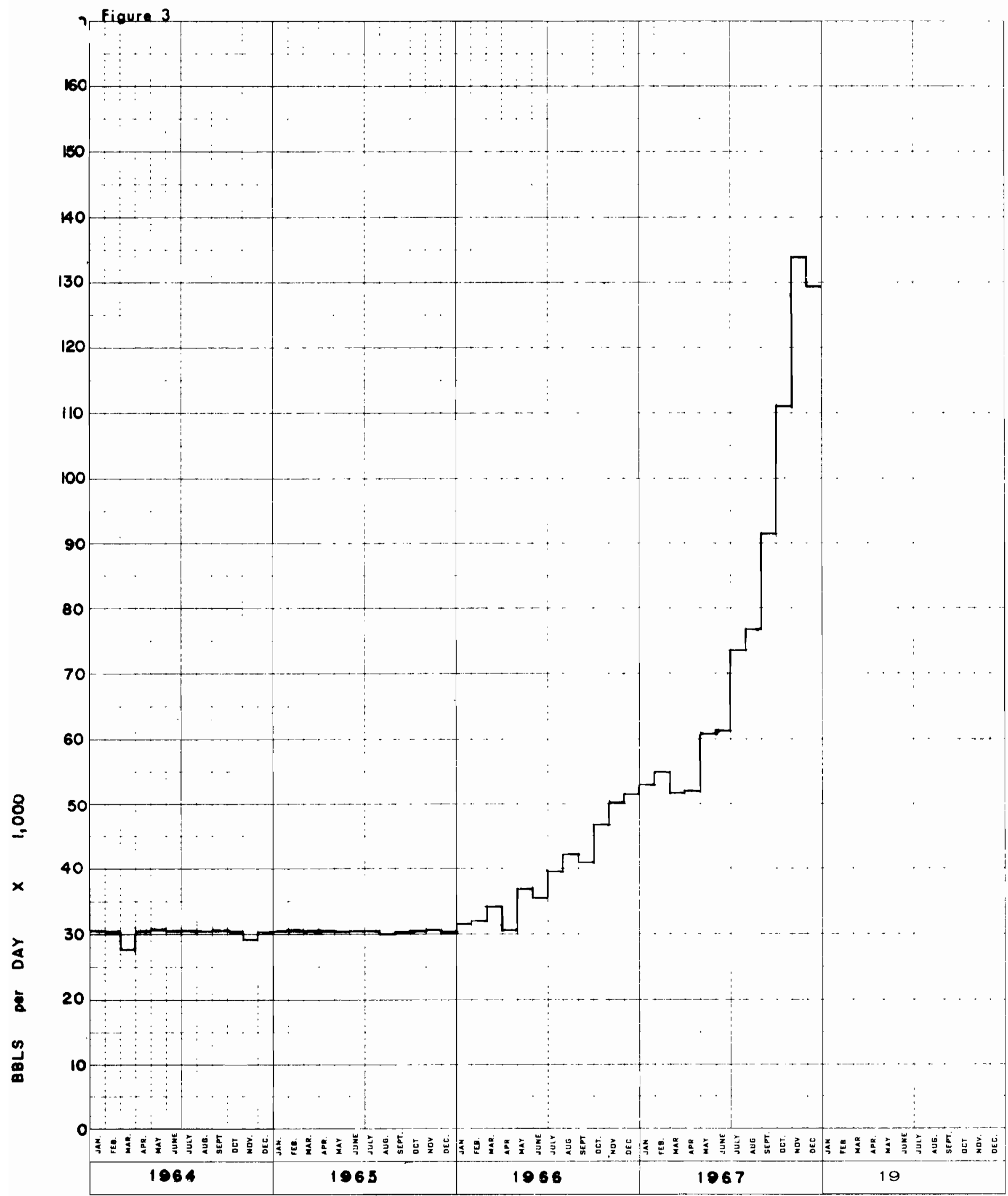


Figure 4

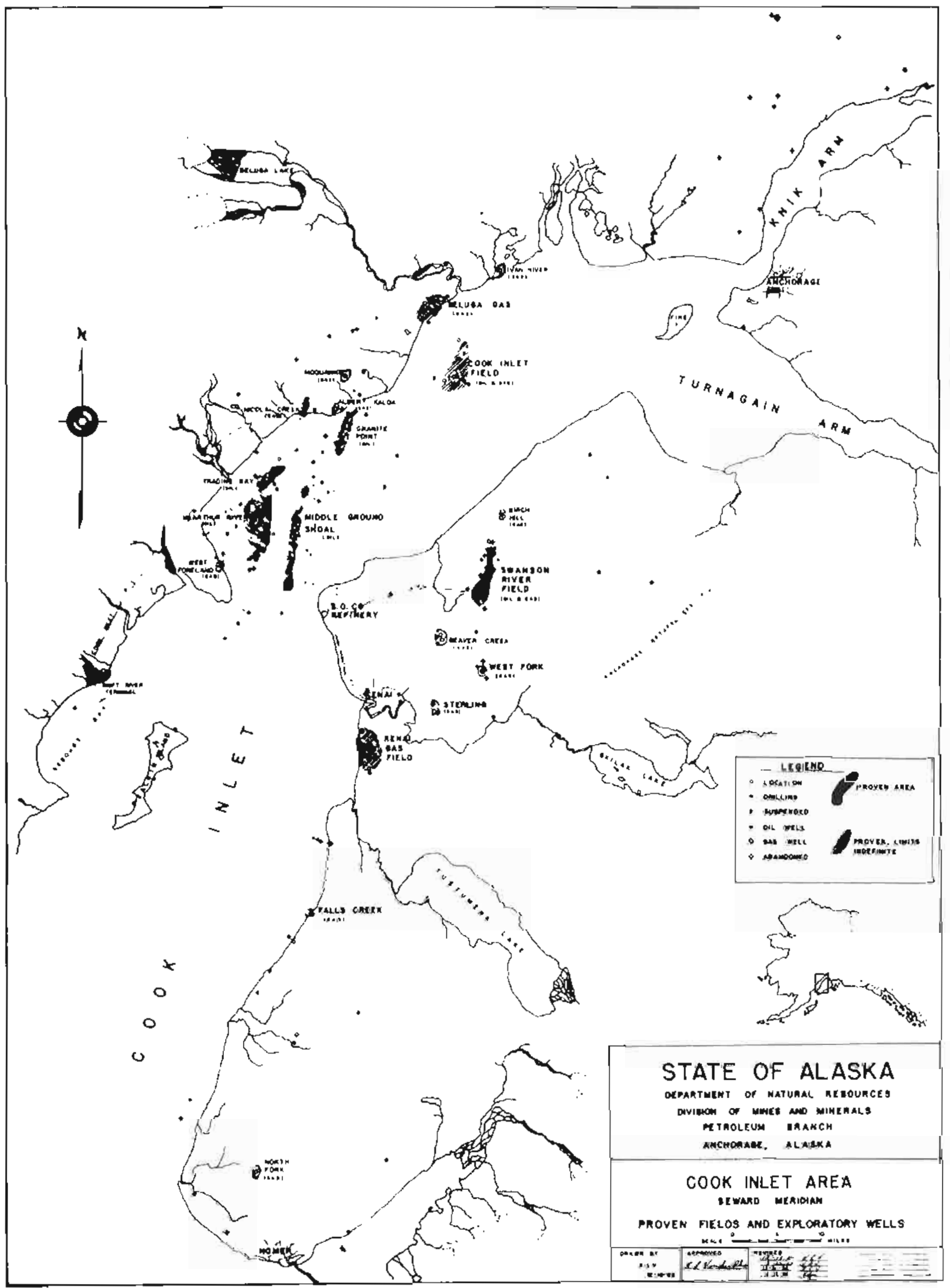


Table V

Summary of Petroleum Industry Statistics*

\begin{tabular}{|c|c|c|c|c|c|}
\hline 1962 & $\underline{1963}$ & 1964 & 1965 & 1966 & 1967 \\
\hline 38 & 25 & 15 & 39 & 64 & 88 \\
\hline 31 & 16 & 16 & 24 & 38 & 27 \\
\hline 10 & 9 & 2 & 13 & $2 n$ & 58 \\
\hline 7 & 8 & 2 & 9 & 15 & 39 \\
\hline 5 & 4 & 5 & 10 & 4 & 4 \\
\hline 21 & 15 & 15 & 7 & 24 & 25 \\
\hline 290,976 & 135,248 & 177,110 & 187,135 & 383,972 & 317,686 \\
\hline 78,619 & 80,337 & 7,499 & 81,236 & 162,513 & 583,030 \\
\hline 369,595 & 215,585 & 184,609 & 268,371 & 546,485 & 901,616 \\
\hline & 7 & 6 & 7 & 14 & 20 \\
\hline 28,107 & 29,424 & 30,285 & 30,400 & 36,958 & 79,226 \\
\hline $1,370,652$ & $1,590,392$ & $1,727,972$ & $3,018,200$ & $3,428,143$ & $3,689,600$ \\
\hline \multirow[t]{7}{*}{$19,550,312$} & $14,035,381$ & $11,589,149$ & $10,184,447$ & $9,264,163$ & $7,111,737$ \\
\hline & & $\$ 5,529,110$ & $\$ 3,425,393$ & $\$ 4,092,165$ & $\$ 3,526,398$ \\
\hline & & $\$ 3,374,603$ & $\$ 3,266,396$ & $\$ 3,600,774$ & $\$ 4,070,451$ \\
\hline & & $\$ 5,511,769$ & $\$ 10,819,708$ & $\$ 7,153,332$ & $\$ 20,256,124$ \\
\hline & & $\$ 1,250,536$ & $\$ 2,656,402$ & $\$ 2,663,482$ & $\$ 2,829,571$ \\
\hline & & $\$ 76,3] 0$ & $\$ 96,233$ & $\$ 941,562$ & $\$ 3,517,136$ \\
\hline & TOTAL & $\$ 15,742,328$ & $\$ 20,264,132$ & $\$ 18,451,315$ & $\$ 34,199,680$ \\
\hline \multirow{4}{*}{$\begin{array}{l}\text { Competitive } \\
\text { competitive } \\
\text { AL } \quad 662,852\end{array}$} & 388,579 & 722,659 & 704,751 & 154,497 & 300,770 \\
\hline & $\frac{143,353}{531,033}$ & $\frac{116,970}{830620}$ & 205,692 & 203,880 & 317,072 \\
\hline & 531,932 & 839,629 & 910,443 & 358,377 & 617,842 \\
\hline & 86,127 & 6,413 & 16,520 & $355,5 \cap 3$ & 33,170 \\
\hline 683,246 & 996,616 & $2,609,714$ & $2,083,010$ & $1,814,989$ & 263,433 \\
\hline
\end{tabular}

Drilling Permits Approved Exploratory Wells Spudded Development Wells Spudded

We1ls Completed (Oil)

Wells Completed (Gas)

We11s Abandoned

Footage Drilled, Exploratory

Footage Drilled, Development

Total Footage Drilled

Average No. Active Rotary Rigs

Average Daily Oil Production

State $O$ \& $G$ lease acreage in effect at year's end

Federal $O \& G$ lease acreage in effect at year's end

Federal payment of $\mathrm{Oil}$ and Gas least rentals

Fecleral paynent of Oil and Gas lease royalty

State Oil and Gas lease bonus

State Oil and Gas lease rental

State Oil and Gas lease royalty

State Oil and Gas lease acreage

issued (does not include trans-

ferred Federal leases)

$$
\text { TOTAL } 662,852
$$

Federal Oil and Gas Icase acreage transferred to State

Federal Oil and Gas lease acreage issued

* See previous reports for years prior to 1962 
(Continued)

Geologic Field Party Months

Seismic Crew Nonths

Gravity Crew Nonths

Magnetometer Crew Nonths

\begin{tabular}{|c|c|c|c|}
\hline 1960 & $10 \leqslant 1$ & 1062 & 1963 \\
\hline 57.5 & $5^{-} \cdot \cdot$ & .13 & 47 \\
\hline 40.0 & $-\Sigma, \ldots$ & 56.23 & 113 \\
\hline 4.9 & 14.5 & 9.5 & 10 \\
\hline
\end{tabular}

1964
22
98
12
2

1965
34
60
3
3

The following figures for 1967 are estimates which may vary from actual amounts because some actual figures are not available at the time of compilation.

Exploration expenditures (includes geological \& geophysical work, exploration drilling, and administrative expense. Does not include money spent for oil \& gas leases acquired by individuals \& out of State companies). (thousands)

Development drilling expenditures (thousands)

Production expenditures (including secondary recovery \& platform construction in year of completion) (thousands)

Refinery construction \& operation expenditures (thousands) (includes petrochemical plants \& marine facilities)

Pipeline construction expenditures (thousands)

Total annual industry expenditures exclusive of marketing $\&$ sales activity (thousands) $\$ 37,805 \$ \$ 42,405 \$ 65,500$

$\begin{array}{llllll}\$ 54,030 & \$ 61,000 & \$ 66,020 & \$ 66,400 & \$ 74,600 \\ \$ 4,635 & \$ 696 & \$ 5,261 & \$ 17,600 & \$ 37,200 \\ \$ 1,594 & \$ 1,758 & \$ 19,250 & \$ 55,800 & \$ 71,800 \\ \$ 5,600 & \$ 2,200 & \$ 2,000 & \$ 6,520 & \$ 34,800 \\ & & \$ 9,550 & \$ 21,080 & \$ 43,800 \\ \$ 75,859 & \$ 65,654 & \$ 102,081 & \$ 169,400 & \$ 262,200\end{array}$

Full time year around employees of oil industry excluding marketing (not excluding all oil field construction activities)

Petroleum marketing personnel

$$
1,508
$$$$
1,599
$$$$
1,680
$$

Cumulative mileage low grade roacs \& seismic trails built by oil industry

Cmulative mileage heavy duty roads built by oil indust?:

Total crude tinroughput - Alaskan Refiner;, barrels (thousands)

$\begin{array}{lllll}2,600 & 5,994 & 6,800 & 6,600 & 6,700\end{array}$


TABLE VI

EXPLORATORY WELLS ACTIVE IN 1967

\begin{tabular}{|c|c|c|c|c|c|c|c|c|c|c|c|c|c|c|c|}
\hline \multirow{3}{*}{$\begin{array}{l}\text { State } \\
\text { Pernit } \\
66.6\end{array}$} & \multirow[b]{2}{*}{ Operator } & \multicolumn{2}{|l|}{ Well } & \multicolumn{5}{|c|}{ Location } & \multicolumn{2}{|c|}{ Date } & \multicolumn{2}{|c|}{ Footage Drilled } & \multirow{2}{*}{$\begin{array}{l}\text { Status } \\
12-31-67\end{array}$} & \multirow{2}{*}{$\begin{array}{l}\text { Initial } \\
\text { Production } \\
\text { Bbls or MCF } \\
\end{array}$} & \multirow[b]{2}{*}{ Remarks } \\
\hline & & Name & No. & $1 / 4$ & Sec. & T. & R. & $\mathrm{BE} \mathrm{M}$ & Spud. & Comp. & T.D. & 1967 & & & \\
\hline & Atlantic Refining & Susie 20 & $T$ & $\mathrm{NE}$ & 22 & $2 \mathrm{~N}$ & 13E & UP & $2-27-66$ & $7=-9-67$ & 13.517 & & Pाल & & \\
\hline $66-40$ & Mobil oil Corp. & Tower $1 / 300$ & 1 & $\mathrm{NE}$ & 10 & $10 \mathrm{~N}$ & $12 \mathrm{~W}$ & $\mathrm{~s}$ & $9-24-66$ & $\begin{array}{r}1-31-07 \\
5-1-67\end{array}$ & $\begin{array}{l}14,879 \\
12,085\end{array}$ & & ${ }_{\mathrm{PEA}}^{P \mathrm{EA}_{1}}-1$ & & \\
\hline $6-43$ & Cities Service oil & Painter Creek & 1 & NW & 14 & $35 \mathrm{~S}$ & 5114 & $\vec{s}$ & $3-26-67$ & $7+16-67$ & 7,012 & 7,912 & $\mathbf{P} Y, \mathrm{~A}$ & & \\
\hline $66-4$ & Pan American & Redoubt Shoal 22064 & 1 & $\mathrm{NE}$ & 26 & $7 \mathrm{~N}$ & $14 \mathrm{~W}$ & $\mathrm{~s}$ & $10-2.66$ & $6-4-67$ & 14,367 & 2,399 & $\mathrm{P} \xi_{1} \mathrm{~A}$ & & \\
\hline $66-54$ & Texaco, Inc. & Point Possession Unit & 1 & $\mathbf{S W}$ & 2 & $10 \mathrm{~N}$ & $7 w$ & $\mathrm{~s}$ & $12-23-66$ & $7-3-67$ & 15,296 & 13,296 & $P F_{1} A$ & & \\
\hline $66-59$ & Pan Anerican & Chui tna River State & 1 & $\mathrm{NE}$ & 15 & $12 \mathrm{~N}$ & $12 \mathrm{~W}$ & s & $1212-66$ & $2-25-67$ & 10,205 & 6,369 & $P F_{A} A$ & & \\
\hline $66-60$ & Marathon Oil co. & Beaver Creek & 1 & SW & 34 & $7 \mathrm{~N}$ & $10 \mathrm{~W}$ & $\underset{P}{S}$ & $\begin{array}{r}1-4-67 \\
12-21-66\end{array}$ & $\begin{array}{l}6-16-67 \\
3-10-67\end{array}$ & 9,134 & 9,134 & $P G A$ & & \\
\hline $\begin{array}{l}66-61 \\
66-63\end{array}$ & $\begin{array}{l}\text { Union Oil co. of } \mathrm{C} \text {. } \\
\text { thent oil co. }\end{array}$ & $\begin{array}{l}\text { Kookpuk } \\
\text { Kalgin Island State }\end{array}$ & $\begin{array}{l}1 \\
2\end{array}$ & $\begin{array}{l}S W \\
\text { SE }\end{array}$ & $\begin{array}{l}19 \\
19\end{array}$ & $\begin{array}{r}11 \mathrm{~N} \\
4 \mathrm{~N}\end{array}$ & $\begin{aligned} 7 E \\
15 W\end{aligned}$ & $\begin{array}{r}\text { UP } \\
\mathrm{S}\end{array}$ & $12-21-66$ & $3-10-67$ & 10,193 & 9,475 & $\begin{array}{l}\mathrm{P} \xi_{\text {A A }} \\
\text { Loc. }\end{array}$ & & \\
\hline 66.64 & Pernzoil Co. & Starichkof State & 1 & $\mathrm{NE}$ & 33 & $3 s$ & $15 \mathrm{~W}$ & $\mathrm{~s}$ & $1-13-67$ & $4-1-67$ & 12,112 & 12,112 & Susp. & & \\
\hline $\begin{array}{l}67-1 \\
67-3\end{array}$ & Shell oil co. & $\begin{array}{l}\text { Cottommood State } \\
\text { Belupa River Unit }\end{array}$ & $\begin{array}{c}1 \\
14-3\end{array}$ & SE & 13 & $11 \mathrm{~N}$ & $14 W$ & s & $1-24-67$ & $2-10-67$ & 4,265 & 4,265 & $P F A$ & & \\
\hline $\begin{array}{l}67-7 \\
67-3\end{array}$ & $\begin{array}{l}\text { Stanalard } 0 \text { il of } C \text {. } \\
\text { Texaco, Inc. }\end{array}$ & $\begin{array}{l}\text { Beluga River thit } \\
\text { Nicolai Creek }\end{array}$ & & & 20 & $12 \mathrm{~N}$ & $12 \mathrm{~W}$ & $\hat{s}$ & $\begin{array}{l}2-1-67 \\
3-19-67\end{array}$ & $\begin{array}{r}9-15-67 \\
5-3-67\end{array}$ & $\begin{array}{r}15,196 \\
8,841\end{array}$ & $\begin{array}{r}15,384 \\
3,333\end{array}$ & $\begin{array}{l}\text { Giti } \\
\text { GSI }\end{array}$ & $5032 \mathrm{MCF} / \mathrm{D}$ & 1/2" bean, T.P. 747 psi - C.P. 815 psi \\
\hline $67-8$ & Shell oil co. & Midule River State & 1 & SW & 5 & $10 \mathrm{~N}$ & $13 \mathrm{~W}$ & $\mathbf{s}$ & $2-15-67$ & $3-8-67$ & 5,287 & 5,287 & $P_{h}, A$ & & \\
\hline $67-9$ & Marathon Oil co. & Beaver Creek & 1-A & SE & 34 & $\pi N$ & $201 \%$ & $\mathrm{~s}$ & $3-13-67$ & $8-1-67$ & 10,296 & 10,609 & CSI & $11,473 \mathrm{MCF} / \mathrm{D}$ & $5 / 8^{\prime \prime}$ bean, T.P. 1592psi \\
\hline $67-11$ & Atlantic Richfield & Prudhoe Bay & 1 & NE & 10 & $11 \mathrm{~N}$ & $14 \mathrm{~F}$ & UP & $4-22-67$ & & 8,593 & 8,593 & Drig. & & \\
\hline $67-12$ & Pan American Pet. & North Mas 18745 & 2 & & 33 & $10 \mathrm{~N}$ & $12 \mathrm{w}$ & $\mathrm{s}$ & $4-20-67$ & $7-31-67$ & $13,9 n 2$ & 13,992 & Susp. & & \\
\hline $67-13$ & Atlantic Richfield & Trading Bay & 1 & SW & 26 & $10 \mathrm{~N}$ & $13 W$ & $\mathrm{~s}$ & $4-20-67$ & $7-5-67$ & 10,050 & 10,950 & Susp. & $1,402 \mathrm{~B} / \mathrm{D}$ & $34.3^{\circ}-20.58-48 / 64^{\prime \prime}$ T.P. $180 \mathrm{psi}$ \\
\hline $67-20$ & Syperior Oil Co. & Three Plile Creek & 1 & NW & 3 & $12 \mathrm{~N}$ & $11 \mathrm{~W}$ & $\mathrm{~s}$ & $7 \cdot 8-67$ & $10-23-67$ & 13,773 & 13,773 & $P q_{A} A$ & & \\
\hline $67-24$ & Nobil oil corp. & Tower & 2 & NW & 22 & $10 \mathrm{~N}$ & $12 \mathrm{~W}$ & 5 & $7-31-67$ & $10-6-67$ & 12,053 & 12,053 & $P P_{A} A$ & & \\
\hline $67-26$ & Marathon 0il co. & Beaver Creek & 2 & NE & 3 & $6 \mathrm{~N}$ & 10w & $s$ & $7-13-67$ & $12-26-67$ & 15,697 & 15,697 & GSI & $7,000 \mathrm{MCF} / \mathrm{D}$ & T.P. $1500 \mathrm{psi}$ \\
\hline $67-29$ & Pan Nerican Pet. Co & . Forelands State Unit & 1 & NE & 22 & $\mathrm{TN}$ & $13 \mathrm{~W}$ & $\mathrm{~s}$ & $6-17-67$ & $8-10-67$ & 12,304 & 12,304 & $P F_{A}$ & & \\
\hline $67-31$ & Pan American Pet. C. & Albert Kaloa & 1 & NE & 26 & $11 \mathrm{~N}$ & $12 W$ & $\mathrm{~s}$ & $7-21-67$ & & 13,600 & 13,818 & Tstg. & & \\
\hline $67-32$ & Thmble oil \& R & Tyonek Reserve " $\mathrm{B}$ " & 1 & $\mathrm{NE}$ & 18 & $11 \mathrm{~N}$ & $11 \mathrm{~W}$ & $s$ & $7-31-67$ & & 15,355 & 15,355 & Drlg. & & \\
\hline $67-34$ & Pan Aurican pet. C. & Tumagain Arm Init & 1 & SE & 9 & $11 \mathrm{~N}$ & SW & $\mathrm{s}$ & $6-25-67$ & $7-17-67$ & 6,325 & 6,325 & $P G A$ & & \\
\hline $67-37$ & Atlantic Richfield & Trading Bay State & 2 & SW & 18 & $10 \mathrm{~N}$ & $12 \mathrm{~W}$ & $\mathrm{~s}$ & $7-8-67$ & $9-24-67$ & 12,410 & 12,410 & $P F_{A}$ & & \\
\hline $67-38$ & Pennzoil co. & Starichkof State Unit & 1 & NE & 22 & 35 & $15 \mathrm{~W}$ & 5 & $8-4-67$ & $9-12-67$ & 8,775 & 8,775 & & & \\
\hline $67-39$ & Shell oil co. & Kustatan Ridge & 1 & $\mathrm{SE}$ & 19 & $9 \mathrm{~N}$ & $14 \mathrm{w}$ & $\mathrm{s}$ & $8-26-67$ & $9-17-67$ & 6,718 & 6,718 & $\mathrm{p}$ & & \\
\hline $67-40$ & Union Oil co. of $\mathrm{C}$. & West Trading Bay State & 1 & $\mathbf{N} \mathbf{W}$ & 4 & $9 \mathrm{~N}$ & $131 \%$ & 5 & $8-2-67$ & $8-24-67$ & 6,528 & 6,528 & $P \xi_{i} \Lambda$ & & \\
\hline $67-43$ & Tenneca Oil co. & State $36465^{\circ}$ & 1 & SW & 34 & $\mathrm{TN}$ & $14 \mathrm{~W}$ & $\mathrm{~s}$ & $8-18-67$ & $11-15-67$ & $13, \cap 63$ & 13,963 & $P \xi_{1} A$ & & \\
\hline $67-44$ & Atlantic Richfield & Middle River St. Init & 1 & $\mathrm{NE}$ & 20 & $10 \mathrm{~N}$ & $131 \mathrm{r}$ & 5 & $8-22-67$ & $10-19-67$ & 10,586 & 10,586 & $\mathrm{P \xi} \mathrm{A}$ & & \\
\hline $67-45$ & Union oil Co. of $C$. & Kenai Decp Unit & 1 & $3 w$ & 6 & $4 \mathrm{~N}$ & $11 w$ & $\mathrm{~s}$ & $0-13-67$ & $11-13-67$ & 9,895 & 10,821 & CSI & (S) $22,500 \mathrm{MCF}$ & :D 1/2" bean - T.P. 2300 \\
\hline $67-5$ & Pan American $P$ & Redoubt Shoal St. 29690 & 01 & $N \mathbf{W}$ & 19 & & & & $8-18-67 \quad \longrightarrow \rightarrow$ & & $13,95 n$ & $1.3,05 n$ & & & \\
\hline 67.5 & inion oil co. of $\mathrm{C}$ & Kasilof thit & 1 & $\mathrm{NE}$ & 30 & $3 \mathrm{~N}$ & $12 \mathrm{~W}$ & $s$ & $n-26-67$ & $10-10-67$ & 5,500 & 5,500 & & & \\
\hline & & & & & & & & & & & & 68 & & & \\
\hline
\end{tabular}


TABLE VII

WELLS ACTIVE IN 1967

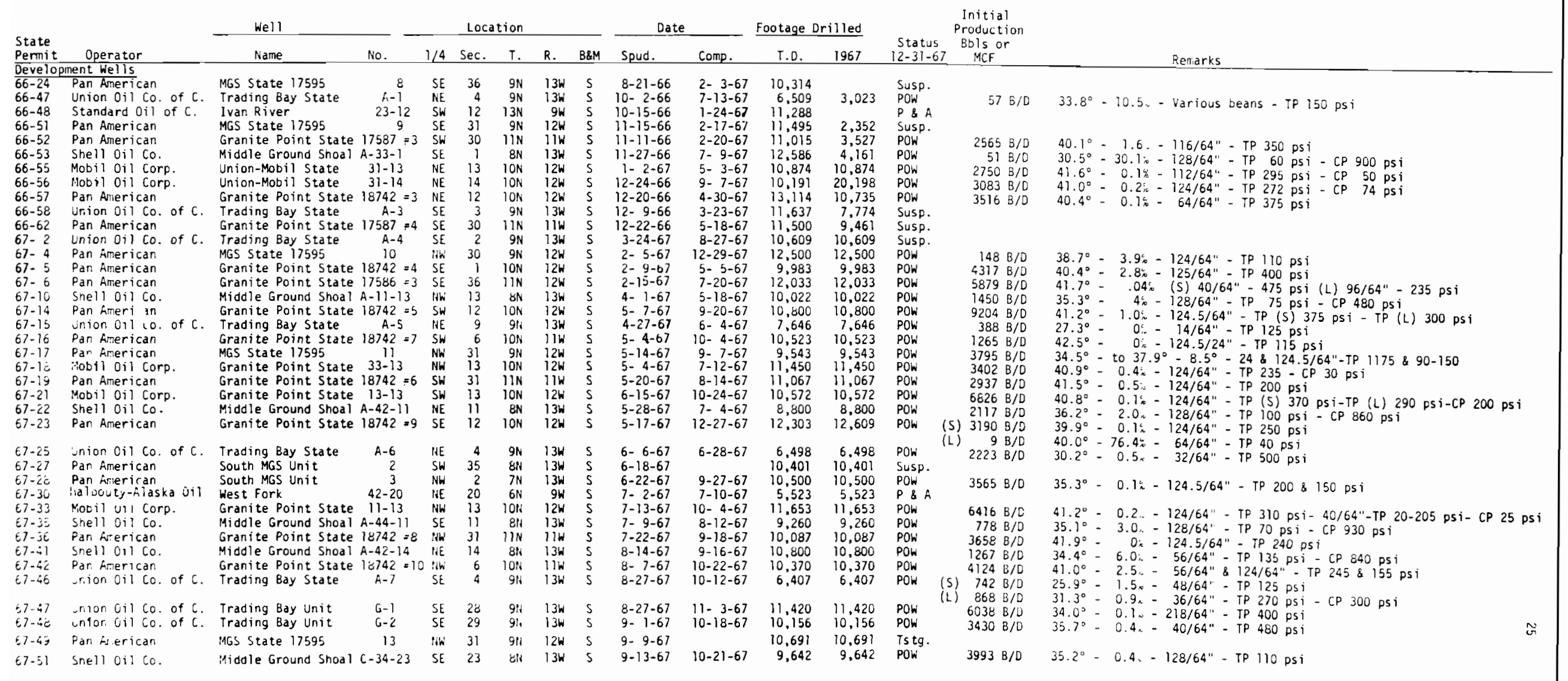


TAPLI. VII

WTLLS MCTITT IN 1967

\begin{tabular}{|c|c|c|c|c|c|c|c|c|c|c|c|c|c|c|c|}
\hline \multirow{4}{*}{ 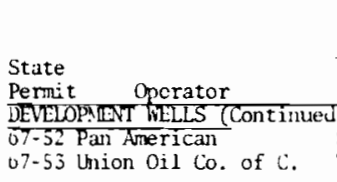 } & \multicolumn{2}{|l|}{ Well } & \multicolumn{5}{|c|}{ Location } & \multicolumn{2}{|c|}{ Datc } & \multicolumn{2}{|c|}{ Footage Drilled } & \multirow{2}{*}{\multicolumn{2}{|c|}{$\begin{array}{l}\quad \text { Initial } \\
\text { Production } \\
\text { Blbls } \\
\text { MCF } \\
\end{array}$}} & & \\
\hline & Name & No. & $1 / 4$ & Sec. & $\mathrm{T}$. & R. & Ben & Spud. & Corm. & T.J). & 1967 & & & & \\
\hline & J) South :IGS Imit & & $5 T^{\prime}$ & . & & $13 W$ & & $n \cdot 2 n-67$ & & & & & & & \\
\hline & Trading Bay State & $A-S$ & Siri & 4 & $x$ & $13 \pi$ & S & $10-11-67$ & $11-11-67$ & 7,083 & 7,083 & Prin & (S) $920 \mathrm{~B} / 1 \mathrm{ll}$ & $33.3^{\circ}-0.1 \%-22 / 64$ & $\begin{array}{l}- \text { TP } 125-200 \mathrm{psi} \\
-\mathrm{TP} 1400 \mathrm{psi}\end{array}$ \\
\hline 07-54 Pan American & Granite Point State & $17586 \$ 4$ & $\mathrm{NT}$ & $j$ & $11 x$ & $12 \%$ & $\mathrm{~s}$ & $n-18-67$ & $11-3 n-67$ & 10,005 & $10,0 n 5$ & & $108 \mathrm{~B} / 1$ & $\begin{array}{l}1.36-18 / 04 " \\
0.6 \%-24 / 64 "\end{array}$ & $\begin{array}{l}\text { - TP } 670 \mathrm{psi} \\
\text { - TP } 190 \mathrm{psi}-124.5 / 64 " .\end{array}$ \\
\hline 07-55 She11 Oil Co. & Middle Ground Shoal & A-12-12 & Nisi & 12 & $3 N$ & $131 \%$ & $\mathrm{~s}$ & $n-2 n-6)$ & $11-1-67$ & 0,650 & 0,650 & POH & $1101 \mathrm{~B} / \mathrm{L}$ & $3.5 \frac{0}{0}-36 / 6.10$ & 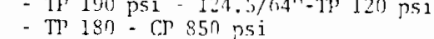 \\
\hline $07-57$.obil oil corp. & Granite Point State & $11-24$ & Mit & 24 & $10 \mathrm{~N}$ & $12 \%$ & s & $10-5-67$ & & 11,800 & 12,529 & Tstg. & & & \\
\hline 7-58 Pan American & South MCS Unit & 4 & Ni & 35 & $8 \mathrm{~N}$ & 1315 & $\mathrm{~s}$ & $10-27-67$ & & $10,55 n$ & in, 550 & Tstg. & & & \\
\hline 67-59 Pan American & Granite Point State & $18742 \# 11$ & $\mathrm{NT}$ & 1 & $10 \mathrm{~N}$ & $121 \%$ & s & $10-12-67$ & & 3,255 & 3,235 & Susp. & & & \\
\hline 07-60 Union Oil $\mathrm{Co}$. of $\mathrm{C}$. & Trading Bay Unit & $r_{1}-3$ & $\mathrm{ATH}$ & 33 & gN & $131 \%$ & $\mathrm{~s}$ & $10-19-67$ & & 10,320 & 13,533 & Nrlg. & & & \\
\hline 67-0l Union Oil Co. of $\mathrm{C}$. & Trading Bay Unit & $c_{1}-4$ & $\mathrm{Ni}$ & 28 & $3 \mathrm{~N}$ & $13 k$ & s & $11-1-6^{2}$ & $12-28-67$ & 10,164 & $1^{n}, 164$ & miti & $0190 \mathrm{~B} / \mathrm{L}$ & $34.7^{\circ}-1.0 \%-90 / 64^{\prime \prime}$ & - IT $300 \mathrm{psi}$ \\
\hline 07-62 bbil Oil corp. & Granite Point State & $44-14$ & $\mathrm{SI}:$ & 14 & $10 \mathrm{~N}$ & $12 \mathrm{Ki}$ & s & $10-24-6^{-}$ & & $1 n, 850$ & in,942 & Tstg. & & $\begin{array}{lll}1.06 & -0 / 04\end{array}$ & 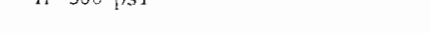 \\
\hline 07-63 Shell Oil co. & Middle Ground Shoal & $c-31-26$ & $\mathrm{~N}$. & 26 & $8 \mathrm{~N}$ & $13 \mathrm{k}^{\prime}$ & $\mathrm{s}$ & $10-22-67$ & $12-9-67$ & 035 & $?, 035$ & pow & $4887 \mathrm{i} 3 / 1)$ & $35.7^{\circ}-11.008-128 / 64^{\prime \prime}$ & - IT $130 \mathrm{n} 5 \mathrm{i}$ \\
\hline 67-64 Union 0il co. of $\mathrm{C}$. & Trading Bay Unit & $k-2$ & $\mathrm{XE}$ & 21 & $9 \mathrm{~N}$ & $131 \%$ & \& & $11-1-67$ & & 11,288 & 11,288 & nrlg. & & 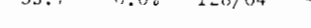 & $-1110 x p 51$ \\
\hline 07-05 Union Oil co. of $C:$ & Trading Bay Unit & $k-1$ & $\mathrm{NF}$ & 19 & $9 \mathrm{~N}$ & 1315 & $\mathrm{~s}$ & $10-26-6 i$ & & 3,224 & 13,224 & Drlg. & & & \\
\hline 67-66 Pan American & Granite Point State & $18742 \# 12$ & $\mathrm{~S}$ & 31 & $11 \mathrm{~N}$ & 1114 & $\mathrm{~s}$ & $10+10-6^{-}$ & & 0,372 & 0,372 & Dr1g. & & & \\
\hline 67-67 Pan American & Granite Point State & $18742 \# 13$ & .76 & 12 & $10 \mathrm{~N}$ & $121 \%$ & s & $7-2.4-67$ & & 9,273 & 10,471 & & & & \\
\hline & Vidule Ground Slroal & $A=33-14$ & & 14 & $8 \mathrm{~N}$ & $13 k$ & s & $11-2-67$ & $12-7-67$ & 10,555 & 10,555 & POW & $2177 \mathrm{~b} / \mathrm{b}$ & $36.4^{\circ}-11.50-128 / 04^{\prime \prime}=$ & - TP ח \\
\hline 7-69 Pan American & South iKS Unit & 7 & Mi & 2 & $7 \mathrm{~N}$ & $13 \%$ & $\mathrm{~s}$ & $11-2-6^{7}$ & & 8,488 & 8,488 & Susp. & & & \\
\hline 7-70 Yobi1 Oil Corp. & Granite Point State & $44-11$ & SE & 11 & $10 \mathrm{~N}$ & $121 \%$ & $s$ & & & & & Loc. & & & \\
\hline 07-71 Uhion 0il co. of $\mathrm{C}$. & Trading Bay Unit & $6-5$ & $\mathrm{NE}$ & 20 & $9 x$ & 1311 & $\mathrm{~s}$ & & & & & Loc. & & & \\
\hline 67-73 Pan American & Granite Point State & 18742 \#14 & $\mathrm{NF}$ & 13 & $11 \mathrm{~N}$ & $111 i$ & $s$ & $11-10-67$ & & 3,230 & 3,230 & Susp. & & & \\
\hline 67-74 Union oil Co. of $\mathrm{C}$. & Trading Bay State & $A-9$ & $\mathrm{NT}$ & 4 & $9 \mathrm{~N}$ & $13 k$ & $s$ & $11-12-67$ & $12-5-67$ & 6,896 & 6,896 & pors & $125 \mathrm{~B} / \mathrm{D}$ & 2b. $.^{0}-2.1:-$ Cas Lift & \\
\hline 67-75 Pan American & South NGS Unit & 9 & $\mathrm{NE}$ & 35 & $8.0^{\circ}$ & $131 \%$ & $\mathrm{~s}$ & $11-22-67$ & & 3,200 & 3,200 & Susp. & & & \\
\hline 67-76 Union 0il Co. of $\mathrm{C}$. & Trading Bay State & $A-10$ & Sir & 4 & $9 \mathrm{~N}$ & $13 k$ & S & $12-5-67$ & & 5.696 & 6,696 & Nrig. & & & \\
\hline 67-77 Pan American & Granite Point State & 18742 \#16 & $\mathrm{NE}$ & 1 & $10 \mathrm{~N}$ & 126 & s & $11-30-67$ & & 5,610 & 8,248 & I) IR. & & & \\
\hline 67-78 Pan American & South MGS Init & 5 & STi & 2 & $7 \mathrm{~N}$ & $13 \pi N$ & $\mathrm{~s}$ & $12-6-67$ & & 3,200 & $3,20 n$ & Susp. & & & \\
\hline 07-79 Shell Oil Co. & Middle Ground Shoal. & $A-14-1$ & SI: & 1 & $8 \mathrm{~N}$ & $13 W$ & $s$ & $12-8-67$ & & 8,430 & $8,43 n$ & Drlg. & & & \\
\hline 07-80 Union 0il Co. of $\mathrm{C}$. & Trading Bay Unit & D-1 & $\mathrm{S} \Gamma$ & 32 & $9 \mathrm{~N}$ & $13 N$ & $s$ & $12-19-67$ & & 2,550 & 2,550 & Drlg. & & & \\
\hline 07-81 Union 0i1 Co. of $\mathrm{C}$. & Trading Bay Unit & $D-2$ & Niv & 5 & $8 \mathrm{~N}$ & $136 \%$ & $\mathrm{~s}$ & $12-23-67$ & & 720 & 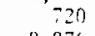 & Drig. & & & \\
\hline 67.82 Pan American & Granite Point State & $18742 \# 15$ & $\mathrm{Mi}$ & 7 & $10 \mathrm{~N}$ & $11 \%$ & 5 & $8-12-67$ & & 8,876 & 8,870 & Drig. & & & \\
\hline 67-85 Shell Oil Co. & Midùle Grownd Siroal & $C-23-26$ & SII & 26 & $8 \mathrm{~N}$ & $13 \mathrm{~K}$ & s & $12-10-67$ & & $?, 19 n$ & $3,10 n$ & Drlg. & & & \\
\hline 67-34 hobil Oil Corp. & Granite Point State & $31-23$ & NI & 23 & $10 N$ & $12 \mathrm{~W}$ & $s$ & & & & & Loc. & & & \\
\hline 07-85 Union 0il Co. of $\mathrm{C}$. & Trading Bay Init & G-6 & $\mathrm{SE}$ & 28 & $2 N$ & 136 & $\mathrm{~s}$ & $12-29-67$ & & 658 & 658 & Drlg. & & & \\
\hline 67-86 Union Oil Co. of $\mathrm{C}$. & Trading Bay init & $k-3$ & $\mathrm{M} M$ & 20 & $9 \mathrm{~N}$ & $131 \%$ & $\mathrm{~s}$ & & & & & Loc. & & & \\
\hline $07-87$ union 0 il $\mathrm{Co}$, of $\mathrm{C}$. & Trading Bay Unit & $k-4$ & Str & 21 & $9 \mathrm{~N}$ & $131 \%$ & $\mathrm{~s}$ & & & & & Loc. & & & \\
\hline 67-so Union oil co. of $c$ & Trading Bay Statc & $A-12$ & NW & 4 & $9 \mathrm{~N}$ & $\begin{array}{l}13 \mathrm{~K} \\
\text { TOTAL }\end{array}$ & $\begin{array}{l}S \\
\text { DEVEI }\end{array}$ & LOPMENT FO & $\begin{array}{l}\text { AGE }= \\
\text { TAAL }=\end{array}$ & $\frac{588,440}{906,134}$ & & Lor. & & & \\
\hline
\end{tabular}


Tabie VIII

\author{
Middle Ground Shoal Field \\ Cook Inlet, Alaska \\ Shell $0 i 1$ Company and \\ Pan American Petroleunl Corporation, Operators
}

$\underline{\text { Location }}$

Discovery Well

Discovery Date

Producing Formation

0 i1

Deepest Test

Wells

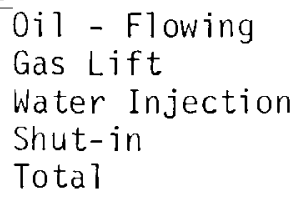

Production Data - 1967

0il Production - bbls. Water Production - bbls. Casinghead Gas - MCF

Cumulative Production 12-31-67

$0 i 1$

Water

Casinghead Gas
TION \& IIN, RIIW \& $12 \mathrm{~W}$, Seward Meridian

Pan American Pet. Corp. - Middle Ground Shoal State No. 1 June 10,1962

Kenai - $5,185^{\circ}-8,100^{\prime}$

Hem7ock - 7,239' - 9,500'

Pan American Pet. Corp. MGS 17595 No. 2 10,862' (VD)

20
10
1
1
32

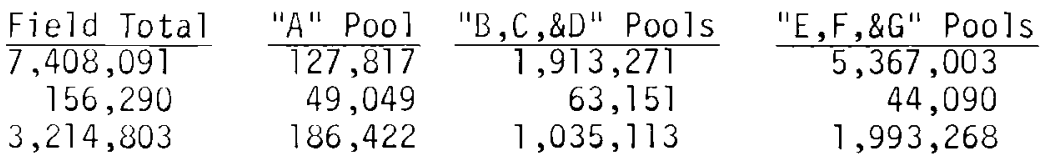

$10,084,636$ bbls. 204,291 bbls.

4,425,193 MCF (base 14.65 psi)
Reservoir Data

Initial Reservoir Pressure

Reservoir Pressure 12/31/67

Saturation Pressure

0 il Gravity

Temperature

Net Pay Thickness

Porosity

Permeability

Connate Water

Original Formation Vol. Factor

Gas/0il Ratio

Productive Area

\begin{tabular}{l} 
Hem1ock Zone \\
\hline 3,655 psi \\
2,500 psi (est) \\
$1,500+$ psi \\
$36^{\circ}-3 \overline{8}^{\circ}$ API \\
$155^{\circ} \mathrm{F}$ \\
$170^{\prime}-550^{\prime}$ \\
$11 \%$ \\
$10 \mathrm{Mds}$. \\
NA \\
NA \\
$430 \mathrm{cf} / \mathrm{bb}$. \\
$4,000 \mathrm{acres}$
\end{tabular}

Kena i Zone

2,000 psi (est)

$1,900+$ ps $i$

$36^{\circ}-38^{\circ}$ API

$125^{\circ} \mathrm{F}$

$250^{\prime}-300^{\prime}$

$16 \%$

100 lids.

NA

NA

$400 \mathrm{cf} / \mathrm{bbl}$.

2,600 acres 
Table IX

Middle Ground Shoal Field

Individual We11 0i1 Production

Well No.

Comp. Date

1965

1966

1967

Cumulative

Pan American Petroleum Corp. - Kenai "A" Sand

$17595 \# 10$ (triple) $12-29-67$

17595 \#11 (triple) 9- 7-67

Total "A" Sand

\begin{tabular}{rr}
19 & 19 \\
127,798 & 127,798 \\
\hline 127,817 & 127,877
\end{tabular}

Pan American Petroleum Corp. - Kenai B, C, \& D Sands

\begin{tabular}{lr}
\hline $17595 \# 5$ & $11-20-66$ \\
$17595 \# 6$ & $6-13-66$ \\
$17595 \# 7$ & $8-9-66$ \\
$17595 \# 10$ & $12-29-67$ \\
$17595 \# 11$ & $9-7-67$ \\
Total B, C, \& D Sands &
\end{tabular}

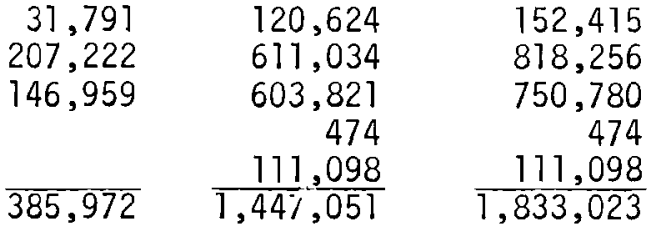

Pan American Petroleum Corp. - Hemlock E, F, \& G Sands

\begin{tabular}{ll}
\hline $17595 \# 4$ & $12-28-66$ \\
$17595 \# 5$ & $11-20-66$ \\
$17595 \# 7$ & $8-9-66$ \\
$17595 \# 10$ & $12-29-66$ \\
$17595 \# 11$ & $9-7-67$ \\
$18746 \# 1$ & (Test) \\
$18746 \# 2$ & (Test) \\
$18746 \# 3$ & $9-27-67$ \\
$18746 \# 4$ & (Test) \\
$18746 \# 6$ & $11-6-67$
\end{tabular}

$\begin{array}{rr}323,507 \\ 5,202 & 135,616 \\ 8,841 & 17,247 \\ & 153 \\ 101,962\end{array}$

323,507

140,818

26,088

153

101,962

1,159

$20,352 \quad 20,352$

413,324

10,879

$\mathrm{m}$

413,324

$\underline{\underline{1,159}} \quad \underline{\underline{14,043}} \quad \underline{\underline{1,297,017}}$

10,879

Total E, F, \& G Sands

267,971

Total Pan American Petroleum Corp.

1,159

400,015

$2,865,881$

$\underline{1,306,213}$

She11 $0 i 1$ Co. - Kenai B, C, \& D Sands

\begin{tabular}{ll}
\hline$A-32-11$ & $9-17-65$ \\
$A-13-1$ & $6-27-66$ \\
A-11-1 & $9-25-66$ \\
Tota1 B, C, \& D Sands &
\end{tabular}

\begin{tabular}{rrrr}
16,602 & 203,551 & 90,957 & 311,110 \\
& 171,465 & 231,531 & 402,996 \\
& 73,051 & 743,732 & $-216,783$ \\
\hline 16,602 & $\frac{748,067}{466,220}$ & $-930,889$
\end{tabular}

Shel1 0 il Co. - Hemlock E, F, \& G Sands

$\begin{array}{lr}A-11-7 & 9-26-66 \\ A-13-1 & 6-27-66 \\ A-33-1 & 7-9-67 \\ A-32-11 & 9-17-65 \\ A-42-11 & 7-4-67 \\ A-43-11 & 6-20-65 \\ A-44-11 & 8-12-67 \\ A-11-12 & 11-24-66 \\ A-12-12 & 11-1-67 \\ A-23-12 & 11-26-65 \\ A-11-13 & 5-18-67 \\ A-32-14 & 2-16-66 \\ A-33-14 & 12-7-67 \\ A-34-14 & 4-14-66 \\ A-42-14 & 9-16-67 \\ C-34-23 & 10-21-67 \\ \text { C-31-26 } & 12-9-67\end{array}$

$\begin{array}{rrr}82,413 & 265,430 & 347,843 \\ 155,095 & 242,054 & 397,149 \\ & 1,058 & 1,058 \\ 63,693 & 52,440 & 116,133 \\ & 334,067 & 334,06 \%\end{array}$

$334,06 \%$

98,072

555,993

48,185

316,821

247,697

814,764

52,438

$362,254 \quad 452,510$

52,438

426,586

609,061

117,322

262,809

$1,035,647$

117,322

262,809

99,282

99,282

Total E, F, \& G Sands

$\overline{9,470} \quad \overline{1,801,292} \quad \frac{99,282}{4,075,992}$

$5,886,694$

Total Shell 0il Co.

Total "A" Sand

Total B, C, \& D Sands

Total E, F, \& G Sands

Total Field Production

$\begin{array}{rrrr}16,602 & 834,039 & 1,913,271 & 2,763,912 \\ \frac{10,569}{27,171} & \frac{1,815,335}{2,649,374} & \frac{5,367,003}{7,408,091} & \frac{7,192,907}{10,084,636}\end{array}$

$\frac{, 817,583}{127,817}$

\begin{tabular}{rrrr}
26,012 & $2,249,359$ & $\frac{4,542,212}{127,817}$ & $6,817,583$ \\
\hline & 127,817
\end{tabular}




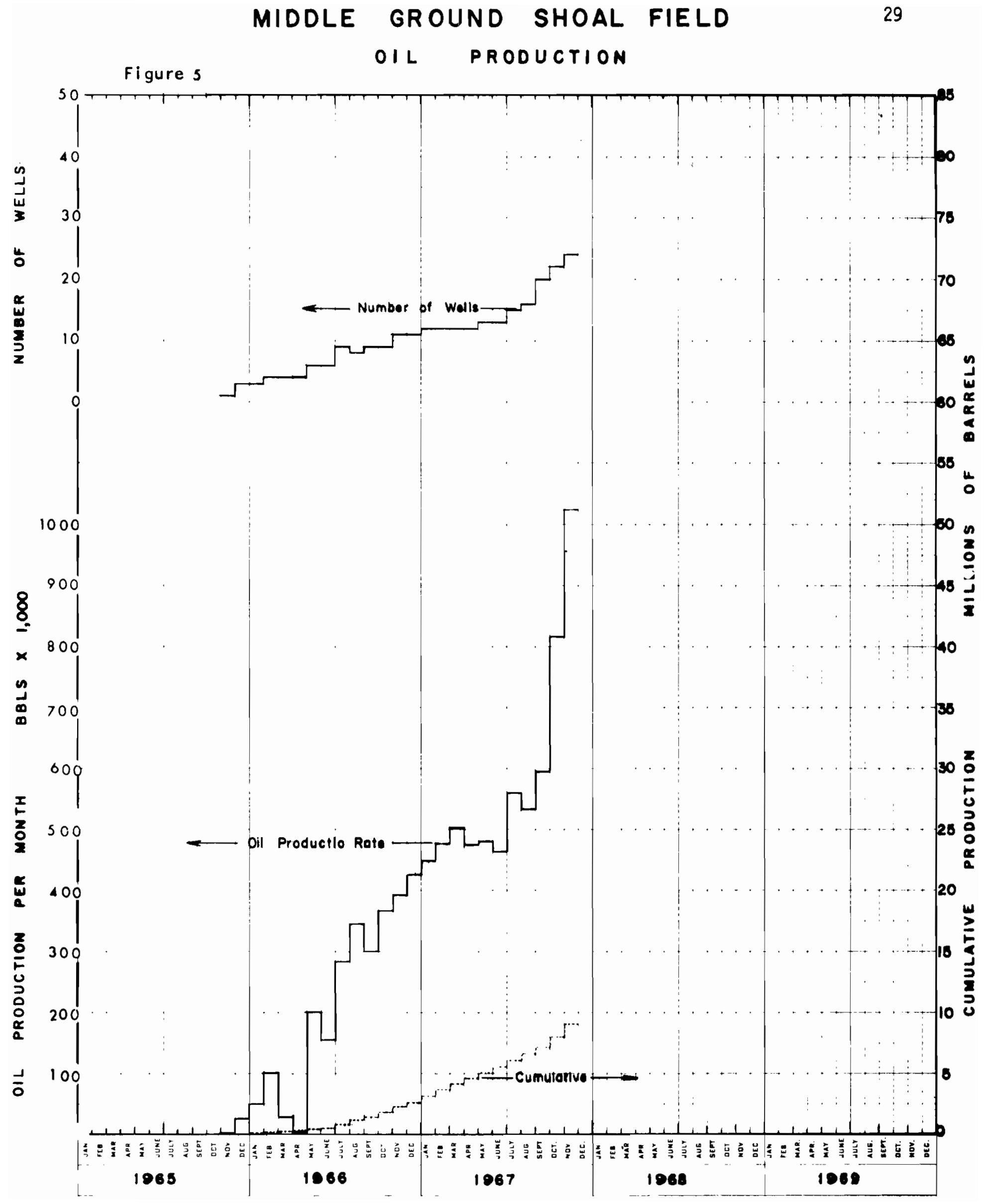


Table $X$

0 il Production By Months

\begin{tabular}{|c|c|c|c|c|c|}
\hline Year & Month & $\begin{array}{l}0 i 1 \text { Prod. } \\
\text { Month-Bb1s. }\end{array}$ & $\begin{array}{l}\text { Oil Prod. } \\
\text { Year-Bb1s. }\end{array}$ & $\begin{array}{c}\text { Cumulative } \\
\text { ail Prod.-Bbls. }\end{array}$ & $\begin{array}{c}\text { Number of } \\
\text { Wells }\end{array}$ \\
\hline $\begin{array}{l}\text { Middle Grou } \\
1965 \\
\text { (corrected) }\end{array}$ & $\begin{array}{l}\text { nd Shoa } \\
\text { Nov. } \\
\text { Dec. }\end{array}$ & $\begin{array}{r}1 \mathrm{~d} \\
1,185 \\
26,012\end{array}$ & 27,197 & 27,197 & $\begin{array}{l}1 \\
3\end{array}$ \\
\hline 1966 & $\begin{array}{l}\text { Jan. } \\
\text { Feb. } \\
\text { Mar. } \\
\text { Apr. } \\
\text { May } \\
\text { June } \\
\text { JuTy } \\
\text { Aug. } \\
\text { Sept. } \\
\text { Oct. } \\
\text { Nov. } \\
\text { Dec. }\end{array}$ & $\begin{array}{r}50,408 \\
101,018 \\
27,394 \\
4,933 \\
196,475 \\
153,794 \\
282,644 \\
345,392 \\
300,572 \\
367,861 \\
391,441 \\
424,897\end{array}$ & $2,646,468$ & 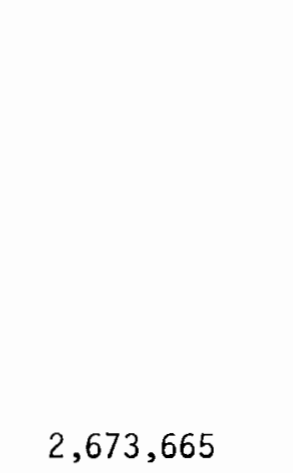 & $\begin{array}{r}4 \\
5 \\
5 \\
6 \\
7 \\
7 \\
11 \\
11 \\
13 \\
13 \\
15 \\
15\end{array}$ \\
\hline 1967 & $\begin{array}{l}\text { Jan. } \\
\text { Feb. } \\
\text { Mar. } \\
\text { Apr. } \\
\text { May } \\
\text { June } \\
\text { July } \\
\text { Aug. } \\
\text { Sept. } \\
\text { Oct. } \\
\text { Nov. } \\
\text { Dec. }\end{array}$ & $\begin{array}{r}448,830 \\
476,051 \\
502,449 \\
475,990 \\
481,479 \\
462,534 \\
560,225 \\
534,751 \\
596,485 \\
815,980 \\
1,023,128 \\
1,030,189\end{array}$ & $7,408,091$ & $10,084,636$ & $\begin{array}{l}16 \\
17 \\
17 \\
16 \\
17 \\
17 \\
19 \\
20 \\
28 \\
28 \\
30 \\
30\end{array}$ \\
\hline
\end{tabular}

Granite Point Field

1965 oct.

$\star 286 \quad 286$

1966 No wells on production

1967

$\begin{array}{lr}\text { Mar. } & 21,943 \\ \text { Apr. } & 72,363 \\ \text { May } & 340,825 \\ \text { June } & 373,471 \\ \text { July } & 536,017 \\ \text { Aug. } & 724,718 \\ \text { Sept. } & 939,024 \\ \text { Oct. } & 1,314,137 \\ \text { Nov. } & 1,381,000 \\ \text { Dec. } & 1,349,566\end{array}$

$\begin{array}{lr}\star 286 & 286 \\ & \\ & 1 \\ & 2 \\ & \\ & 4 \\ & 4 \\ & 6 \\ 7,053,064 & 7 \\ & 17 \\ & 14 \\ & 15 \\ & 17\end{array}$

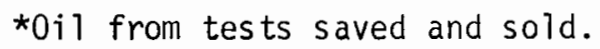




\author{
Table XI \\ Granite Point Field \\ Cook Inlet, Alaska
}

Mobi1 $0 i 1$ Corporation and

Pan American Petroleum Corporation, Operators

\section{Location}

Discovery Well

Discovery Date

Producing Formation

Deepest Test

Wells

0i1 - Flowing

Gas Lift

Shut-in

Production Data - 1987

0il Production

Water Production

Gas Production - with oil

Cumulative Production 12/31/67

$0 i 1$

Water

Gas - with oil

\section{Reservoir Data}

Initial Reservoir Pressure Reservoir Pressure 12/31/67

Saturation Pressure

$0 i 1$ Gravity

Formation Temperature

Net Pay Thickness

Porosity

Permeability

Gas-0il Ratio

Productive Area
T10 \& $11 \mathrm{~N}, \mathrm{R} 11$ \& $12 \mathrm{~W}$, Seward Meridian

Mobil 0 il Corporation, Granite Point \#1

June 9,1965

Kenai Zone - 7,750'-10,200'

Pan American Pet. Corp., Tyonek State 17587 No. 2 - 12,288'

16

0

0

$7,053,064$ bbls.

50,483 bbls.

4,889,550 MCF (base 14.65 psi)

$10,084,636 \mathrm{bb} 7 \mathrm{~s}$.

$194,916 \mathrm{bb} 7 \mathrm{~s}$.

4,889,550 MCF (base 14.65 psi)

4,116 psi a 8,500'

2,600 psi (est)

2,400 psi +

$47^{\circ}-44^{\circ}$ AP $\overline{\mathrm{I}}$

$135^{\circ}-170^{\circ} \mathrm{F}$

$250^{\prime}-600^{\prime}$

$14 \%$

$10 \mathrm{Mds}$.

$960 \mathrm{SCF} / \mathrm{STB}$

3,200 Acres 
OIL PRODUCTION

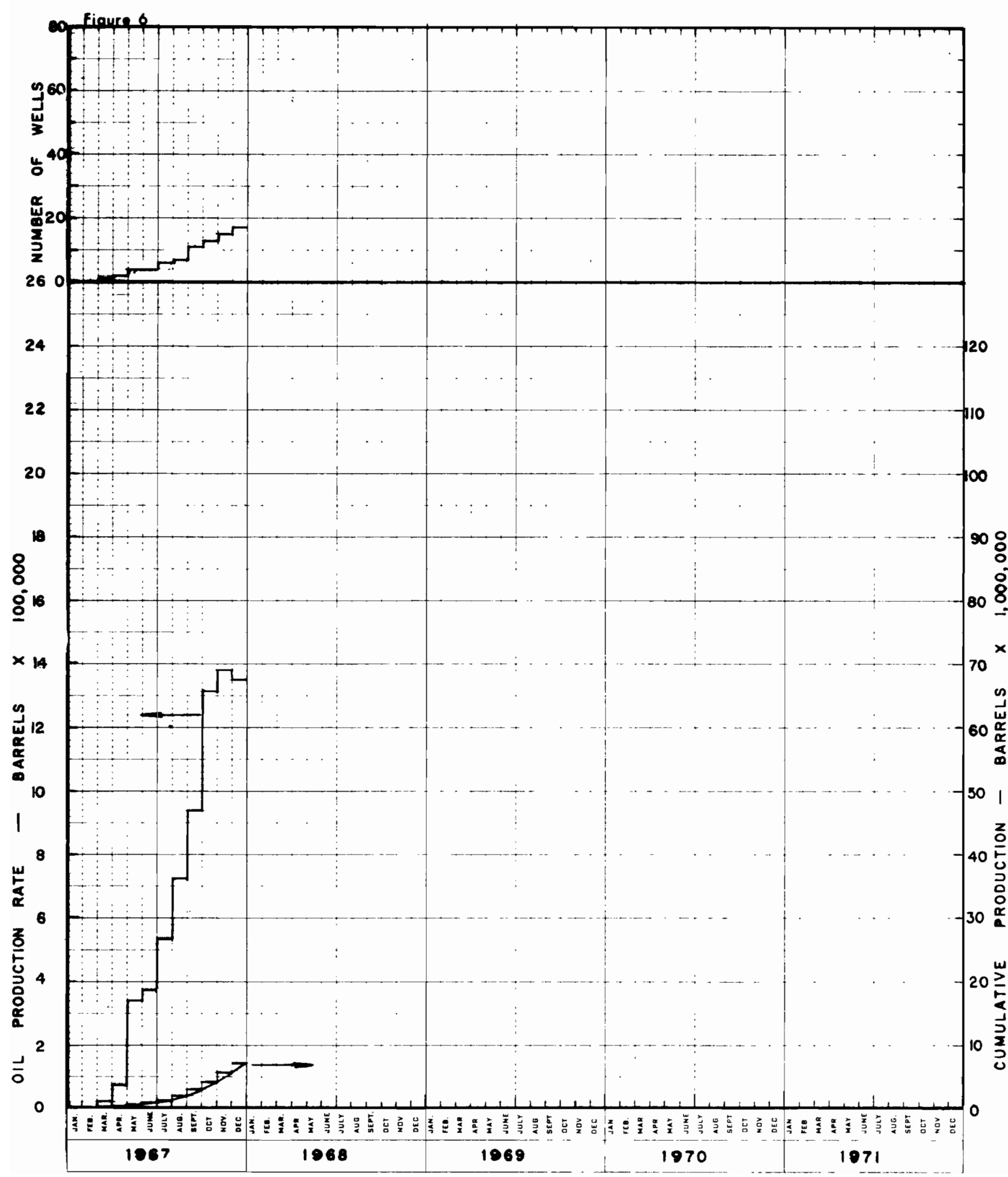


Table XII

Granite Point Field

Individual Well 0 il Production

\begin{tabular}{|c|c|c|c|}
\hline Well No. & Comp. Date & 1965 & 1967 \\
\hline $\begin{array}{l}\text { Mobil 0i1 Corp. - Middle Kenai S } \\
\# 31-13 \\
\# 33-13 \\
\# 31-14 \\
\# 11-13 \\
\# 13-13 \\
\text { Total Middle Kenai Sands }\end{array}$ & $\begin{array}{r}\text { Sands } \\
5-11-67 \\
9-7-67 \\
10-4-67 \\
10-24-67\end{array}$ & & $\begin{array}{r}786,102 \\
566,112 \\
311,278 \\
485,774 \\
330,240 \\
2,479,506\end{array}$ \\
\hline $\begin{array}{l}\text { Mobil 0il Corp. - Hemlock Sands } \\
\text { \#11-13 } \\
\text { Total Hemlock Sands }\end{array}$ & $10-4-67$ & & $\begin{array}{r}2,764 \\
2,764 \\
\end{array}$ \\
\hline Total Mobil 0 il Corp. & & & $2,482,270$ \\
\hline $\begin{array}{l}\text { Pan American Petroleum Corp. - M } \\
18742 \# 2 \\
17587 \# 3 \\
18742 \# 4 \\
18742 \# 3 \\
17586 \# 3 \\
18742 \# 6 \\
18742 \# 5 \\
18742 \# 8 \\
18742 \# 7 \\
18742 \# 10 \\
17586 \# 4 \\
18742 \# 9 \\
\text { Total Pan American - Middle Kena }\end{array}$ & 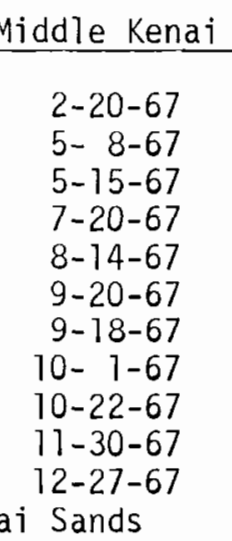 & 286 & $\begin{array}{r}582,048 \\
831,985 \\
651,864 \\
751,154 \\
315,023 \\
718,264 \\
245,704 \\
104,822 \\
263,382 \\
68,497 \\
38,051 \\
4,570,794\end{array}$ \\
\hline $\begin{array}{l}\text { Total Field - Middle Kenai Sands } \\
\text { Total Field - Hemlock Sands }\end{array}$ & & & $\begin{array}{r}7,050,300 \\
2,764 \\
\end{array}$ \\
\hline Total Field Production & & 286 & $7,053,064$ \\
\hline
\end{tabular}


Table XIII

\author{
Swanson River Field \\ Kenai Peninsula, Alaska \\ Standard $0 i 1$ Company of California, \\ Western Operations, Inc., Operator
}

Location

Discovery Well

Discovery Date

Producing Formation

$0 i 1$

Gas

Deepest Test

Wells
T7N \& 8N R9W, Seward Meridian

SRU 34-10

August 24, 1957

Hemlock Zone - 10,150'-11,700'

Kenai - 3000' $-5800^{\prime}$

SCU 22A-32-14,796'

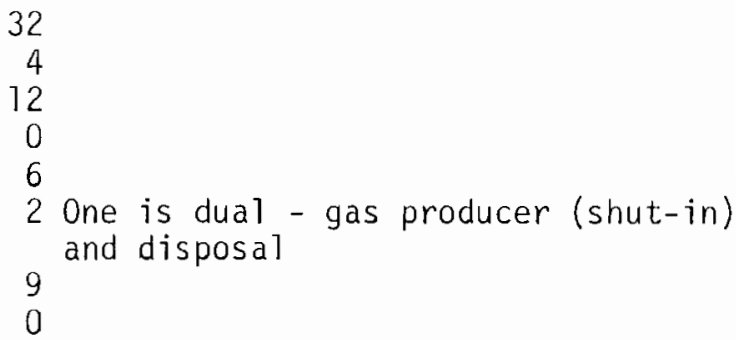

$12,980,482$

$12,257,904$

$13,541,028$ (base 14.65 psi)

0

Gas Production - with oil

Cumulative Production 12-31-67

$0 i 1$

Water

Gas - with oil

Gas - gas wells

Reservoir Data - Hemlock Zone

Initial Reservoir Pressure

Reservoir Pressure 12-31-67

Saturation Pressure

0i] Gravity

Temperature

Net Pay Thickness

Porosity

Permeability

Connate Water

Original Formation Vol. Factor

Gas-0il Ratio

Participating Area
74,951,420 bb 1s.

$8,277,867$ bbls.

32,375,631 MCF (base 14.65 psi)

$11,839,352$ MCF (base 14.65 psi)

$$
\begin{aligned}
& 5,650 \mathrm{psi} \\
& 4,800 \mathrm{ps} i \\
& 1,000-1,400 \\
& 30.0^{\circ}-37.8^{\circ} \text { API } \\
& 180^{\circ} \mathrm{F} \\
& 8^{\prime}-300^{\prime} \\
& 18-26 \% \\
& 0-3,275 \mathrm{Mds} . \\
& 40 \% \\
& 1.12 \\
& 139-753 \mathrm{SCF} / \mathrm{STB} \\
& 6,245 \text { acres }
\end{aligned}
$$




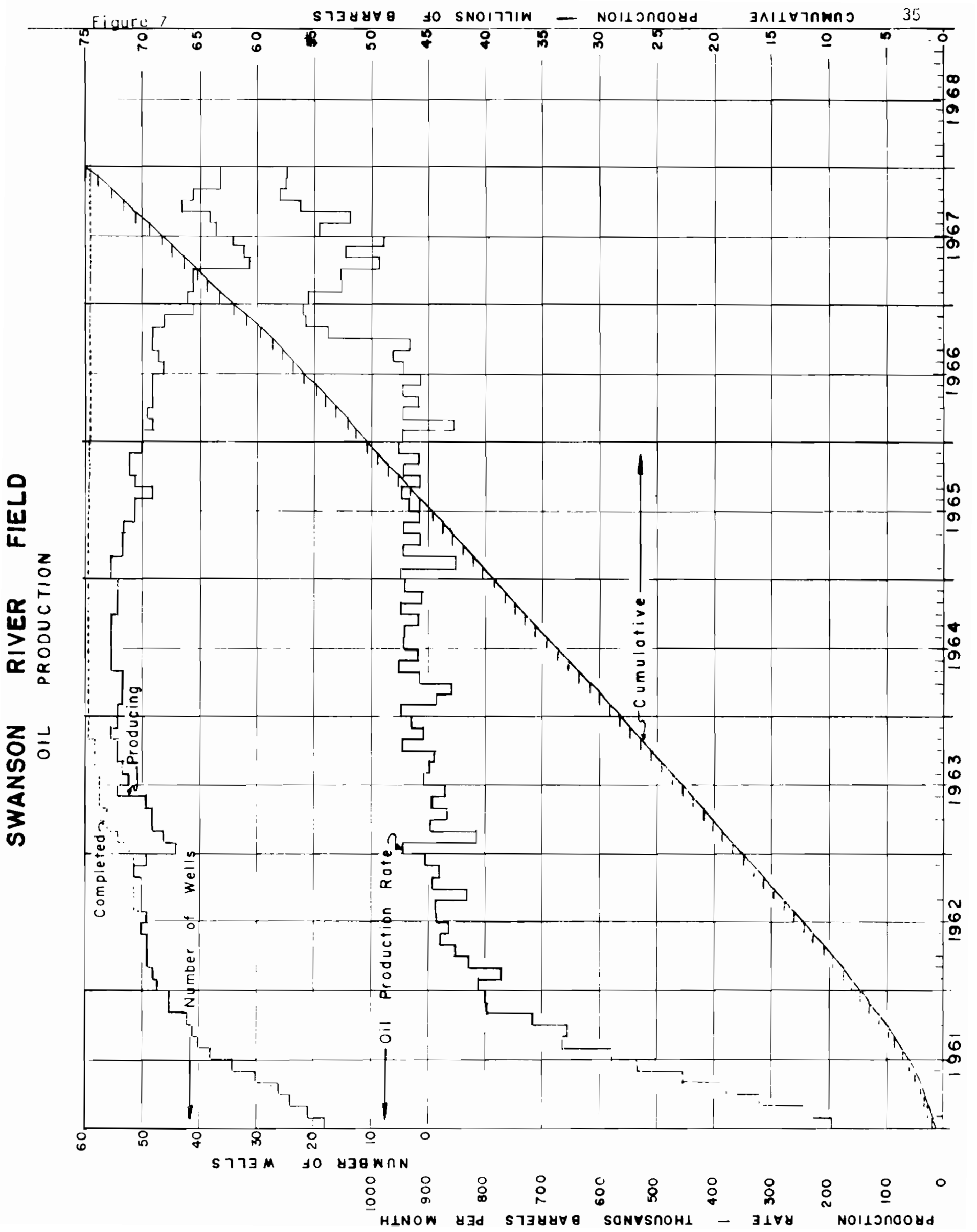


Table XIV

0i) Production By Months

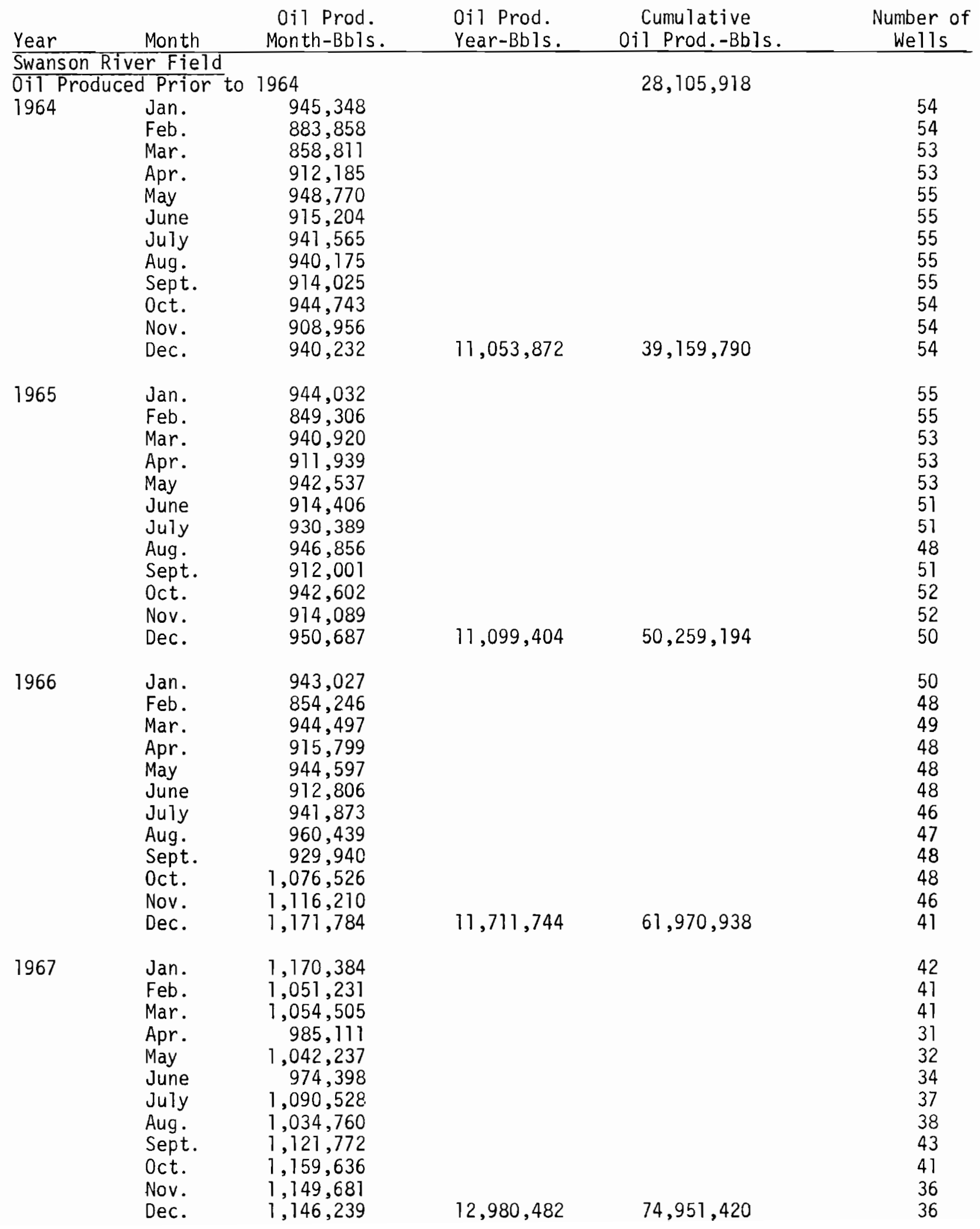


Swanson River Field

Individual Well 0il Production Statistics

Swanson River Unit

0i1 Production

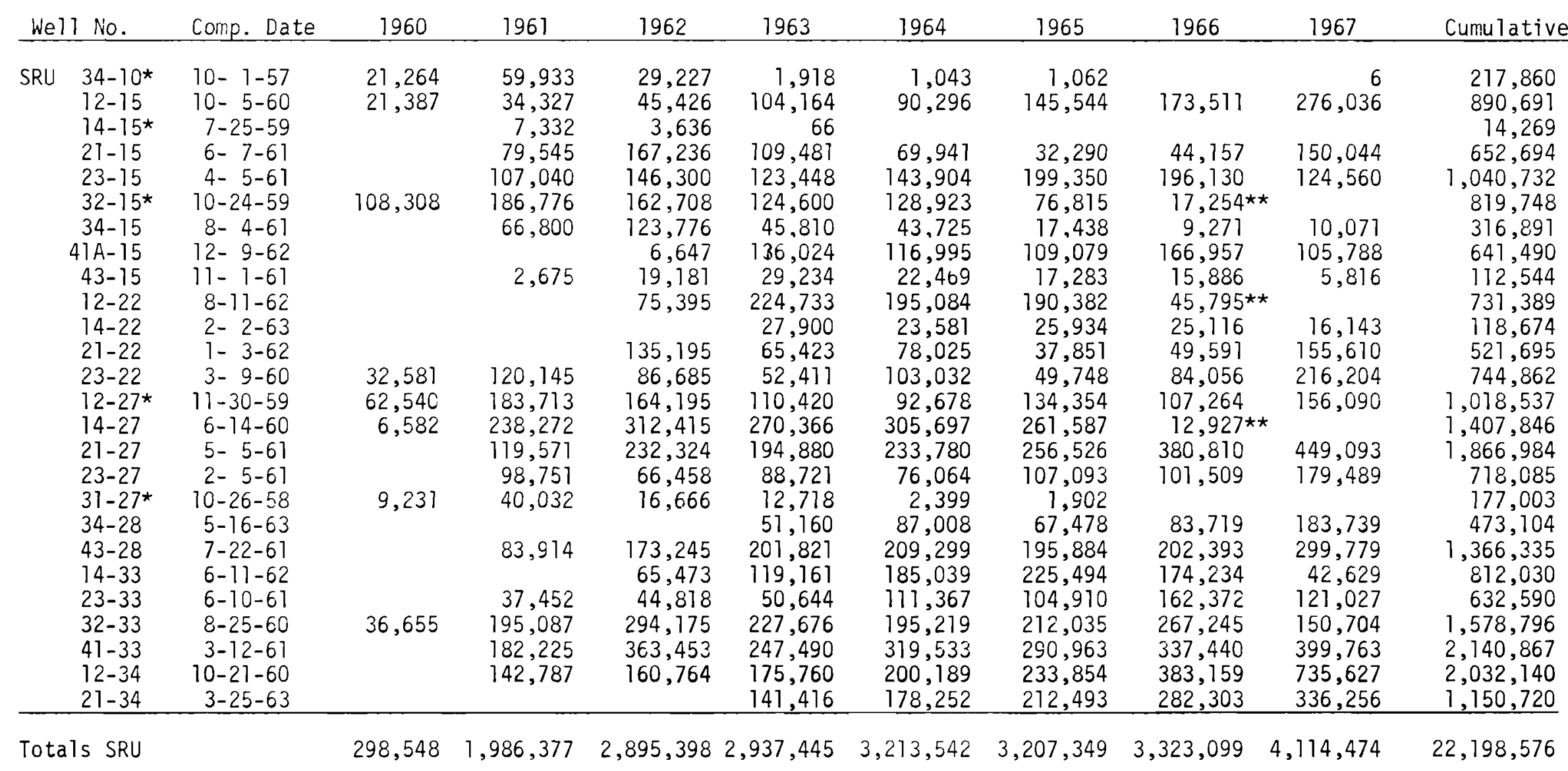

* Produced prior to 1960 - Total 222,344 bbls.

$\star \star$ Converted to gas injection wells 
Table XVI

Swanson River Field

Individual We11 011 Production Statistics

Soldotna Creek Unit

0 il Production

\begin{tabular}{|c|c|c|c|c|c|c|c|c|c|c|c|}
\hline & $12-3$ & $3-23-61$ & & $201, \overline{829}$ & 241,286 & 163,195 & $25 \overline{7}, 984$ & 161,443 & 222,425 & 415,061 & $1,663,223$ \\
\hline & $14-3$ & $8-2-61$ & & 66,962 & 127,862 & 117,475 & 86,680 & 3,618 & 5,748 & 4,938 & 407,283 \\
\hline & $21-3$ & $1-10-62$ & & & 253,580 & 199,238 & 123,737 & 181,022 & 195,677 & 1,130 & 954,384 \\
\hline & $23-3$ & $11-12-63$ & & & & 13,452 & 70,841 & 27,018 & 16,010 & 4,411 & 131,732 \\
\hline & $12-4$ & $4-8-61$ & & 206,870 & 315,308 & 448,589 & 337,381 & 375,646 & 485,699 & 669,360 & $2,838,853$ \\
\hline & $14-4$ & $8-13-60$ & 83,768 & 305,632 & 406,899 & 474,979 & 433,066 & 288,242 & $5,108^{\star}$ & & $1,997,694$ \\
\hline & $21-4$ & $17-5-61$ & & 29,605 & 215,841 & 255,905 & 286,564 & 328,064 & 377,862 & 76,796 & $1,570,637$ \\
\hline & $23-4$ & $6-1-61$ & & 175,004 & 370,777 & 472,517 & 375,279 & 439,947 & 504,484 & 711,969 & $3,049,977$ \\
\hline & $32-4$ & $6-5-60$ & 74,003 & 317,492 & 398,596 & 425,710 & 381,621 & 335,942 & 491,669 & $493,749 *$ & $2,918,782$ \\
\hline & $34-4$ & $11-26-60$ & & 246,747 & 355,595 & 437,873 & 387,460 & 418,123 & 517,342 & 794,498 & $3,157,638$ \\
\hline & $47-4$ & $3-21-60$ & 97,543 & 301,962 & 280,253 & $\star$ & & & & & 679,758 \\
\hline & $43-4$ & $5-14-61$ & & 181,890 & 330,931 & 327,105 & 317,238 & 339,799 & 330,536 & 461,835 & $2,288,334$ \\
\hline & $32-5$ & $3-16-62$ & & & 29,946 & 67,290 & 102,183 & 85,880 & 54,551 & 20,777 & 360,527 \\
\hline & $34-5$ & $10-9-61$ & & 36,817 & 262,993 & 420,163 & 343,194 & 538,278 & 375,809 & 207,379 & $2,178,633$ \\
\hline & $41-5$ & $9-20-61$ & & $4], 594$ & 116,075 & 102,833 & 173,845 & 119,476 & 369,247 & 337,091 & $1,260,161$ \\
\hline & $43-5$ & $5-28-61$ & & 91,504 & 127,021 & 219,725 & 233,669 & 285,977 & 369,227 & 314,396 & $1,641,519$ \\
\hline & $21-8$ & $2-5-63$ & & & & 164,964 & 260,072 & 260,038 & 1,888 & 99,286 & 786,248 \\
\hline & $41-8$ & $5-74-61$ & & 119,506 & 355,493 & $87,270^{\star}$ & & & & & 562,269 \\
\hline & $32-8$ & $2-22-61$ & & 130,883 & 194,030 & 204,460 & 242,599 & 347,609 & 470,841 & 705,204 & $2,295,626$ \\
\hline & $34-8$ & $1-28-62$ & & & 76,666 & 105,205 & 162,007 & 151,421 & 166,656 & 161,832 & 823,787 \\
\hline & $43-8$ & $7-24-61$ & & 161,965 & 154,901 & 120,657 & 153,302 & 106,952 & 198,451 & 152,302 & $1,048,530$ \\
\hline & $12-9$ & $10-9-60$ & & 275,247 & 236,586 & * & & & & & 511,833 \\
\hline & $14-9$ & $10-26-60$ & & 181,998 & 195,332 & 314,525 & 292,351 & 368,675 & 488,640 & 369,416 & 2,21 \\
\hline & $21-9$ & $7-19-61$ & & 137,997 & 353,681 & 390,562 & 411,971 & 471,527 & 402,019 & 94,793 & $2,262,550$ \\
\hline & $23-9$ & $7-17-61$ & & 115,333 & 331,927 & 341,006 & 215,240 & 260,544 & 245,718 & 65,323 & $1,575,091$ \\
\hline & $32-9$ & $1-16-61$ & & 185,766 & 282,033 & 373,733 & 387,757 & 457,009 & 466,487 & 748,555 & $2,901,340$ \\
\hline & $34-9$ & $11-8-61$ & & 26,014 & 194,171 & 259,224 & 285,562 & 227,058 & 340,128 & 339,124 & $1,671,281$ \\
\hline & $41-9$ & $6-11-61$ & & 118,240 & 260,815 & 288,681 & 277,325 & 100,998 & 103,800 & 426,917 & $1,576,776$ \\
\hline & $43-9$ & $7-5-63$ & & & & 83,926 & 164,747 & 4,127 & 14,922 & 562 & 303,284 \\
\hline & $21-16$ & $7-8-63$ & & & & 110,491 & 281,106 & 242,731 & 314,278 & 341,170 & $1,289,776$ \\
\hline & $34-33$ & $11-12-60$ & 4,137 & 281,661 & 243,842 & 227,676 & 256,625 & 320,377 & 368,870 & 474,430 & $2,177,618$ \\
\hline & $43-33$ & $3-8-61$ & & 164,602 & 290,258 & 260,721 & 233,723 & 374,104 & $146,859 *$ & & $1,470,267$ \\
\hline & $14-34$ & $12-23-60$ & & 243,004 & 361,114 & 329,369 & 306,201 & 270,410 & 337,695 & 338,704 & $2,186,497$ \\
\hline Tot: & 5 SCU & & 259,451 & $4,340,124$ & $7,363,712$ & $7,802,519$ & $7,840,330$ & $7,892,055$ & $8,388,646$ & $8,866,008$ & $52,752,845$ \\
\hline & $S$ SRU & & 298,548 & $\mathrm{~T}, 986,377$ & $2,895,398$ & $2,937,445$ & $3,213,542$ & $3,207,349$ & $3,323,099$ & $4,114,474$ & $22,198,576$ \\
\hline & & & & & & & & & & & \\
\hline Field & Totals & 222,344 & 557,999 & $6,326,501$ & $10,259,110$ & $10,739,964$ & $11,053,872$ & $11,099,404$ & $11,711,745$ & $12,980,482$ & $74,951,421$ \\
\hline
\end{tabular}

* Converted to gas injection well 
Table XVII

Swanson River Injection Project

Injection Statistics*

Gas Injection Started November, 1962

Pressure Base 14.65 psi

\begin{tabular}{|c|c|c|c|c|c|}
\hline Date & & $\begin{array}{l}\text { Number } \\
\text { Injection Welis }\end{array}$ & $\begin{array}{l}\text { MCF Injected } \\
\text { in Month }\end{array}$ & $\begin{array}{c}\text { MCF from } \\
\text { Kenai (Included) }\end{array}$ & $\begin{array}{l}\text { Cumulative } \\
\text { MCF Injected }\end{array}$ \\
\hline $\begin{array}{l}1962, \\
\text { Tota) } 1962\end{array}$ & $\begin{array}{l}\text { Nov. } \\
\text { Dec. }\end{array}$ & $\begin{array}{l}1 \\
2\end{array}$ & $\begin{array}{r}33,547 \\
225,068 \\
258,615\end{array}$ & & $\begin{array}{r}33,547 \\
258,615 \\
\end{array}$ \\
\hline $\begin{array}{l}1963 \\
\text { Tota1 } 1963\end{array}$ & $\begin{array}{l}\text { Jan. } \\
\text { Feb. } \\
\text { Mar. } \\
\text { Apr. } \\
\text { May } \\
\text { June } \\
\text { July } \\
\text { Aug. } \\
\text { Sept. } \\
\text { Oct. } \\
\text { Nov. } \\
\text { Dec. }\end{array}$ & $\begin{array}{l}2 \\
2 \\
3 \\
3 \\
3 \\
3 \\
3 \\
3 \\
3 \\
3 \\
3 \\
3\end{array}$ & $\begin{array}{r}306,038 \\
360,840 \\
537,487 \\
474,304 \\
549,380 \\
621,453 \\
739,865 \\
664,394 \\
586,840 \\
640,587 \\
508,440 \\
488,185 \\
6,477,813\end{array}$ & & $\begin{array}{r}564,653 \\
925,493 \\
1,462,980 \\
1,937,285 \\
2,486,665 \\
3,108,118 \\
3,847,982 \\
4,512,376 \\
5,099,217 \\
5,739,803 \\
6,248,243 \\
6,736,428 \\
\end{array}$ \\
\hline $\begin{array}{l}1964, \\
\text { Total } 1964\end{array}$ & $\begin{array}{l}\text { Jan. } \\
\text { Feb. } \\
\text { Mar. } \\
\text { Apr. } \\
\text { May } \\
\text { June } \\
\text { July } \\
\text { Aug. } \\
\text { Sept. } \\
\text { Oct. } \\
\text { Nov. } \\
\text { Dec. }\end{array}$ & $\begin{array}{l}3 \\
3 \\
3 \\
3 \\
3 \\
3 \\
3 \\
3 \\
3 \\
3 \\
3 \\
3\end{array}$ & $\begin{array}{r}524,308 \\
471,948 \\
523,147 \\
574,812 \\
550,281 \\
516,281 \\
502,187 \\
454,445 \\
421,230 \\
404,523 \\
360,719 \\
316,093 \\
5,619,974\end{array}$ & & $\begin{array}{r}7,260,736 \\
7,732,685 \\
8,255,832 \\
8,830,644 \\
9,380,924 \\
9,897,205 \\
10,399,392 \\
10,853,837 \\
11,275,067 \\
11,679,590 \\
12,040,309 \\
12,356,402 \\
\end{array}$ \\
\hline Total 1965 & $\begin{array}{l}\text { Jan. } \\
\text { Feb. } \\
\text { Mar. } \\
\text { Apr. } \\
\text { May } \\
\text { June } \\
\text { July } \\
\text { Aug. } \\
\text { Sept. } \\
\text { Oct. } \\
\text { Nov. } \\
\text { Dec. }\end{array}$ & $\begin{array}{l}3 \\
3 \\
3 \\
3 \\
3 \\
3 \\
2 \\
3 \\
3 \\
3 \\
3 \\
3\end{array}$ & $\begin{array}{r}362,977 \\
323,497 \\
399,684 \\
391,990 \\
391,166 \\
358,970 \\
360,770 \\
353,179 \\
346,959 \\
355,218 \\
379,158 \\
819,742 \\
4,843,310\end{array}$ & $\begin{array}{c}\text { Dented Gas } \\
\text { from } \\
\text { Kenai Field } \\
66,694 \\
\frac{586,844}{653,538}\end{array}$ & $\begin{array}{l}12,719,379 \\
13,042,876 \\
13,442,560 \\
13,834,550 \\
14,225,716 \\
14,584,686 \\
14,945,457 \\
15,298,635 \\
15,645,595 \\
16,000,812 \\
16,379,970 \\
17,199,712 \\
\end{array}$ \\
\hline
\end{tabular}


Table XVII (Cont.)

Swanson River Injection Project Injection Statistics

Gas Injection Started November, 1962

\begin{tabular}{|c|c|c|c|c|c|}
\hline Date & & $\begin{array}{l}\text { Number of } \\
\text { Injection Wells }\end{array}$ & $\begin{array}{l}\text { MCF Injected } \\
\text { in Month }\end{array}$ & $\begin{array}{l}\text { MCF from } \\
\text { Kenai (Included) }\end{array}$ & $\begin{array}{l}\text { Cumulative } \\
\text { MCF Injected }\end{array}$ \\
\hline 1966, & $\begin{array}{l}\text { Jan. } \\
\text { Feb. } \\
\text { Mar. } \\
\text { Apr. } \\
\text { May } \\
\text { June } \\
\text { Juiy } \\
\text { Aug. } \\
\text { Sept. } \\
\text { Oct. } \\
\text { Nov. } \\
\text { Dec. }\end{array}$ & $\begin{array}{l}3 \\
3 \\
4 \\
4 \\
7 \\
8 \\
8 \\
7 \\
6 \\
6 \\
7 \\
7\end{array}$ & $\begin{array}{r}850,803 \\
756,898 \\
844,509 \\
807,343 \\
2,727,741 \\
3,836,009 \\
3,888,203 \\
3,177,489 \\
2,276,498 \\
3,690,283 \\
3,151,895 \\
2,761,950 \\
28,769,621\end{array}$ & $\begin{array}{r}624,246 \\
583,590 \\
672,788 \\
636,066 \\
2,656,545 \\
3,515,596 \\
3,702,589 \\
2,840,720 \\
2,021,062 \\
3,348,035 \\
2,816,373 \\
2,025,233 \\
25,443,413\end{array}$ & $\begin{array}{l}18,050,515 \\
18,807,413 \\
19,651,922 \\
20,459,265 \\
23,187,006 \\
27,023,015 \\
30,911,218 \\
34,088,707 \\
36,365,205 \\
40,055,488 \\
43,207,383 \\
45,969,333 \\
\end{array}$ \\
\hline Total 1967 & $\begin{array}{l}\text { Jan. } \\
\text { Feb. } \\
\text { Mar. } \\
\text { Apr. } \\
\text { May } \\
\text { June } \\
\text { July } \\
\text { Aug. } \\
\text { Sept. } \\
\text { Oct. } \\
\text { Nov. } \\
\text { Dec. }\end{array}$ & $\begin{array}{l}7 \\
7 \\
2 \\
7 \\
7 \\
8 \\
8 \\
8 \\
8 \\
8 \\
8 \\
9\end{array}$ & $\begin{array}{l}2,587,361 \\
3,145,312 \\
1,423,962 \\
2,292,776 \\
3,790,716 \\
3,477,430 \\
3,611,951 \\
3,991,258 \\
3,833,969 \\
3,803,712 \\
4,048,136 \\
4,739,250 \\
40,145,833\end{array}$ & $\begin{array}{r}1,973,323 \\
2,495,978 \\
1,035,369 \\
1,936,293 \\
3,310,543 \\
2,878,970 \\
2,831,400 \\
3,164,227 \\
2,858,382 \\
2,756,672 \\
2,733,715 \\
2,798,504 \\
30,773,376\end{array}$ & $\begin{array}{l}48,556,694 \\
51,702,006 \\
53,125,968 \\
55,418,744 \\
59,209,460 \\
62,686,890 \\
66,298,841 \\
70,290,099 \\
74,124,068 \\
77,927,780 \\
81,975,916 \\
86,115,166 \\
\end{array}$ \\
\hline
\end{tabular}


TABLE: XVIII

Trading Bay Field

Kenai Peninsula, Alaska

Union Oi1 Company of California, Operator

Location

Discovery Wel1

Discovery Date

Producing Formation

Oil

Gas

Deepest Test

We11s

$\begin{array}{ll}\text { Oil - } & \text { Flowing } \\ & \text { Gas Lift } \\ & \text { Shut-in } \\ \text { Gas - Producing } \\ \text { Tota1 }\end{array}$

Production Data - 1967

Oil Production

Water Production

Gas Production - with oil

Gas Production - gas wells

Cumulative Production 12/31/67

Oil

Water

Gas - with oil

Gas - gas wells

Reservoir Data

Initial Reservoir Pressure

Reservoir Pressure 1/31/67

Saturation Pressure

Oil Gravity

Temperature

Net Pay Thickness

Porosity

Permeability

Gas/Oil Ratio

Productive Area
Sec. $3 \& 4$, T9N, R13W

Union Oil Company, Trading Bay 1-A

June 1, 1965

Upper Kenai

Upper Ilemlock

Upper Kenai

Atlantic Richfield Trading Bay State \#1 - 10,950'

5

3

2

1

11

Middle Kenai Zone $\quad$ lemlock Zone

135,634 bisls. $\quad 501,360$ ints

158 b) $1 \mathrm{~s}$. $620 \mathrm{bbls}$.

204,027 MCF(basc 14.65psi) 469,725 MCF(base 14.65psi) $47,268 \mathrm{MCF}$

0

135,634 lh1s.

158 bbls.

$204,927 \mathrm{MCF}$ (hase $14.65 \mathrm{psi}$ ) $470,240 \mathrm{MCF}$ (hase $14.65 \mathrm{psi}$ ) $47,268 \mathrm{MCF}$ 0

Middle Kenai Zone

$2,163 \mathrm{psi}$

2,140 (est)

1,921 psi

$25.6^{\circ} \mathrm{API}$

$112^{\circ} \mathrm{F}$

$100^{\prime}-1,000^{\prime}$

NA

$\mathrm{N} \Lambda$

$268 \mathrm{cf} / \mathrm{bl} \mathrm{l}$.

480 acres
Ilemlock Zone

$2,745 \mathrm{psi}$

2,675 (est)

1,622 psi

$31.1^{\circ} \mathrm{API}$

$136^{\circ} \mathrm{F}$

$300^{\prime}$

$14.6 \%$

$10 \mathrm{Mds}$.

$318 \mathrm{cf} / \mathrm{bl} 1$

640 acres 
TRADING BAY FIELD

OIL PRODUCTION

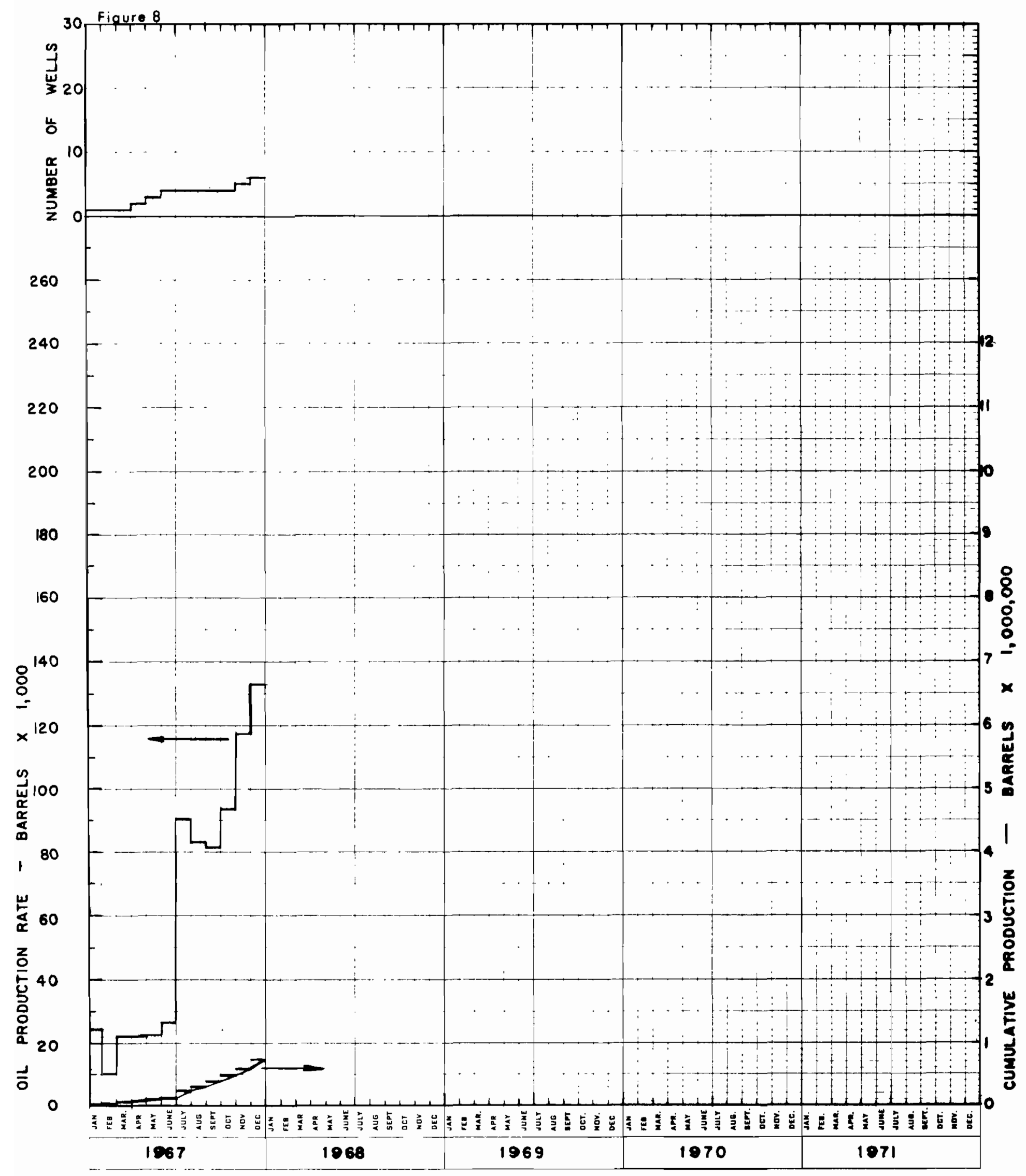


TABLE XIX

Trading Bay Field

Individual Well Oil Production

\begin{tabular}{|c|c|c|c|c|c|}
\hline Well No. & Comp. Date & 1965 & 1966 & 1967 & Cumulative \\
\hline Union Oil Co. - Middle Kenai & Zone & & & & \\
\hline $\mathrm{A}-2$ (dual) & $12-6-66$ & Gas on $\mathrm{v}$ & & & \\
\hline$A-3$ & $4-24-67$ & & (on tests) & 28 & 28 \\
\hline$A-4$ & $5-30-67$ & & & 1,858 & 1,858 \\
\hline A- 5 & $6-4-67$ & & & 54,695 & 54,695 \\
\hline A-7 (dual) & $10-12-67$ & & & 55,517 & 55,517 \\
\hline A-8 (dual) & $11-11-67$ & & & 23,536 & 23,536 \\
\hline Total Middle Kenaj Zone & & & & 135,634 & 135,634 \\
\hline \multicolumn{6}{|l|}{ Union Oil Co. - Hemlock Zone } \\
\hline $1-A$ & Test. & 1,684 & & & 1,684 \\
\hline A-2 (dual) & $12-6-66$ & & & 229,609 & 229,609 \\
\hline$A-4$ & 7-67-Test & & & 41 & 41 \\
\hline$A-6$ & $6-28-67$ & & & 306,648 & 306,648 \\
\hline$A-1$ & $11-3-67$ & & & 538 & 538 \\
\hline A-7 (dua1) & $10-12-67$ & & & 52 & 52 \\
\hline A-8 (dual) & $11-11-67$ & & & 53,007 & 53,007 \\
\hline$A-9$ & $12-5-67$ & & & 1,465 & 1,465 \\
\hline Total liemlock Zone & & 1,684 & & 501,360 & 593,044 \\
\hline Total Trading Bay Field & & $\overline{1,684}$ & & 726,994 & 728,678 \\
\hline
\end{tabular}

TABLE XX

Oil Production By Months

\begin{tabular}{|c|c|c|c|c|c|}
\hline Year & Month & $\begin{array}{l}\text { Oil Prod. } \\
\text { Month-Bbls. }\end{array}$ & $\begin{array}{l}0 \text { Oil Prod. } \\
\text { Year-Bbls. }\end{array}$ & $\begin{array}{l}\text { Cumulative } \\
\text { Oi1 Prod. Bh1s. }\end{array}$ & $\begin{array}{r}\text { Numbe } \\
\text { Well } \\
\end{array}$ \\
\hline$\frac{\text { TRADING BAY }}{1965}$ & $\frac{\text { FIELD }}{\text { July }}$ & $* 1,684$ & 1,684 & 1,684 & 1 \\
\hline 1966 & No we & roduction & & & \\
\hline 1967 & $\begin{array}{l}\text { Jan. } \\
\text { Feb. } \\
\text { Mar. } \\
\text { Apr. } \\
\text { May } \\
\text { June } \\
\text { July } \\
\text { Aug. } \\
\text { Sept. } \\
\text { Oct. } \\
\text { Nov. } \\
\text { Dec. }\end{array}$ & $\begin{array}{r}24,388 \\
10,099 \\
22,349 \\
22,381 \\
22,977 \\
26,436 \\
90,352 \\
83,031 \\
81,611 \\
93,792 \\
116,690 \\
132,906\end{array}$ & 727,012 & 728,696 & $\begin{array}{l}1 \\
1 \\
1 \\
2 \\
3 \\
4 \\
5 \\
4 \\
4 \\
4 \\
5 \\
6\end{array}$ \\
\hline
\end{tabular}

MCARTHIUR RIVER FIELD

1965 No we11s on production

1966 No wells on production

1967

$$
\begin{array}{lr}
\text { Oct. } & 59,010 \\
\text { Nov. } & 338,972 \\
\text { Dec. } & 350,833
\end{array}
$$

748,815

748,815

1

$\frac{2}{3}$

1967 Totals

$28,917,464 \quad 93,566,917$

*Oil from tests saved and sold 
TABLE: XXI

\author{
McArthur River Field \\ Trading Bay Unit \\ Union Oil Company of California, Operator
}

Location

Discovery Well

Discovery Date

Producing Formation

leepest Test

We11s

Oil - Flowing

Gas Lift

Shut-in

Total

Production Data - 1967

Oil production

Water Production

Gas Production - with oil

Cumulative Production 12/31/67

O11

Water

Gas

Reservoir Data

Initial Reservoir Pressure

Reservoir Pressure 12/31/67

Saturation Pressure

Oil Gravity

Temperature

Net Pay Thickness

Porosity

Permeability

Gas/Oil Ratio

Productive Area
T8N 9N - R13W, Seward Meridian

Union Oil Company Grayling No. 1-^

October 24,1965

lemlock Zone

She11 Forelands Channel State No. 1-A $11,786^{\prime}$ Drilled. 11,736' TVI)

3
0
0
3

748,815 bbls.

771 bhis.

$219,915 \mathrm{MCF}$ (hase $14.65 \mathrm{psi}$ )

$750,846 \mathrm{bbls}$

771 blols.

$210,915 \mathrm{MCF}$ (base $14.65 \mathrm{psi}$ )

4,300 psi

4,225 psi (est.)

1,782 psi

$35^{\circ}-36^{\circ}$ API

$154^{\circ}-168^{\circ} \mathrm{F}$

$300^{\prime}$

NA

$\mathrm{NA}$

$306 \mathrm{cf} / \mathrm{bl}) 1$

10,000 acres

TABLI: XXII

McArthur River Ficld

Individual Well Oil Production

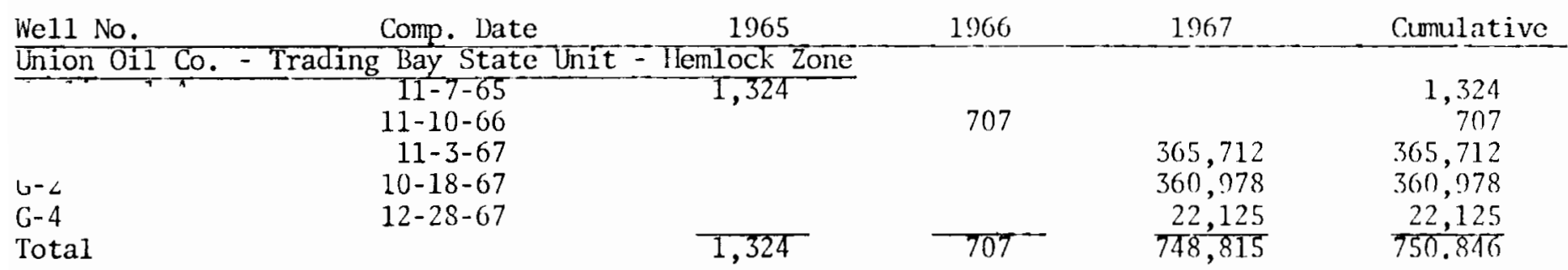




\section{MCARTHUR RIVER FIELD}

OIL PRODUCTION

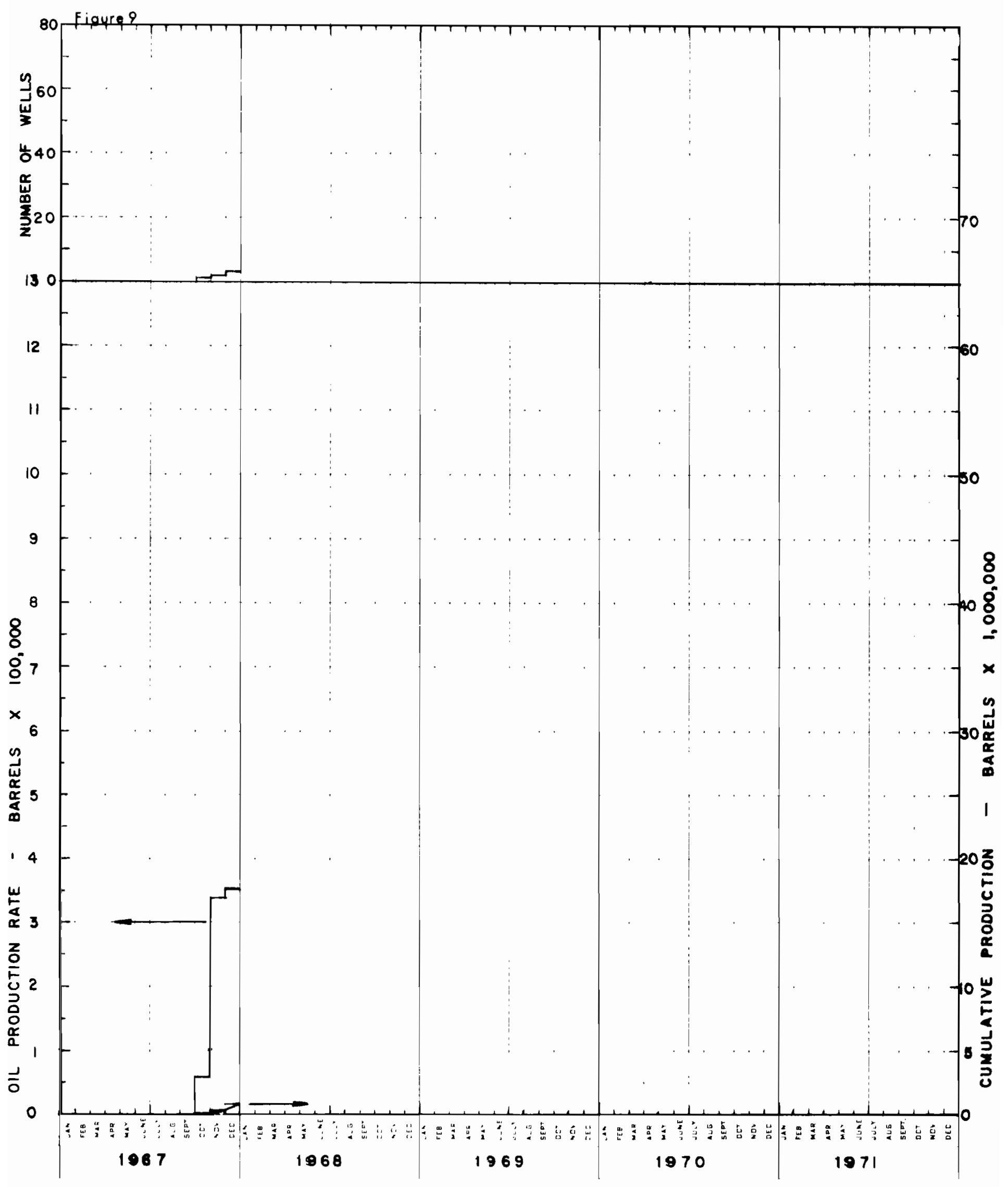


Table XXVI

Kenai Deep Gas Field

Kenai Peninsula, ATaska

Union $0 i 1$ Company of California, Operator

Location

Discovery Well

Discovery Date

Producing Formation

Deepest Test

Wells

Producing

Shut-in

Reservoir Data

Initial Reservoir Pressure

Gas Gravity

Temperature

Net Pay Thickness

Porosity

Permeability

Connate Water

Developed Area
Sec. 6, T4N - R11W, Seward Meridian

Kenai Deep Unit No. 1

November 13, 1967

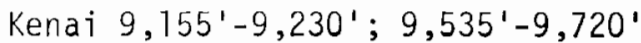

Kenai Unit No. 14-6 drilled to $15,047^{\prime}$

0

1

4,401 psi@9,632'

0.56

143

NA

NA

NA

NA

640 acres

Production Statistics

Year Producing Shut-in Water (bbls) Year $\underline{\text { Cumulative }}$

1967

0

0

8,000

8,000 Test Prod. 
Table XXVII

Sterling Gas Field

Kenai Peninsula, ATaska

Union $0 i 1$ Company of California, Operator

Location

Discovery Well

Discovery Data

Producing Formation

Deepest Test

Wells

Producing

Shut-in

Reservoir Data

Initial Reservoir Pressure

Average Reservoir Pressure

Gas Gravity

Temperature

Net Pay Thickness

Porosity

Permeability

Connate Water

Developed Area
Sec. 15, T5N-R10W, Seward Meridian

No. $23-15$

August 4, 1961

Kenai 5,250'-54' (Perforations)

No. $23-15-14,832^{\prime}$

1

1

$2,200-2,300$ psi

2,200 psi

.569

$108-110^{\circ} \mathrm{F}$

$10-30$

1,500 acres

Production Statistics

Number of Wells

\begin{tabular}{|c|c|c|c|c|c|}
\hline Year & Producing & Shut-in & Water & Year & Cumlulative \\
\hline 1962 & 1 & 0 & 0 & 25,186 & 25,186 \\
\hline 1963 & 1 & 1 & 0 & 45,724 & 70,910 \\
\hline 1964 & 1 & $i$ & 0 & 58,383 & 129,293 \\
\hline 1965 & 1 & 1 & 0 & 120,319 & 249,612 \\
\hline 1966 & $i$ & $j$ & 0 & 157,490 & 407,102 \\
\hline 1967 & $i$ & $i$ & 0 & 179,544 & 586,646 \\
\hline
\end{tabular}




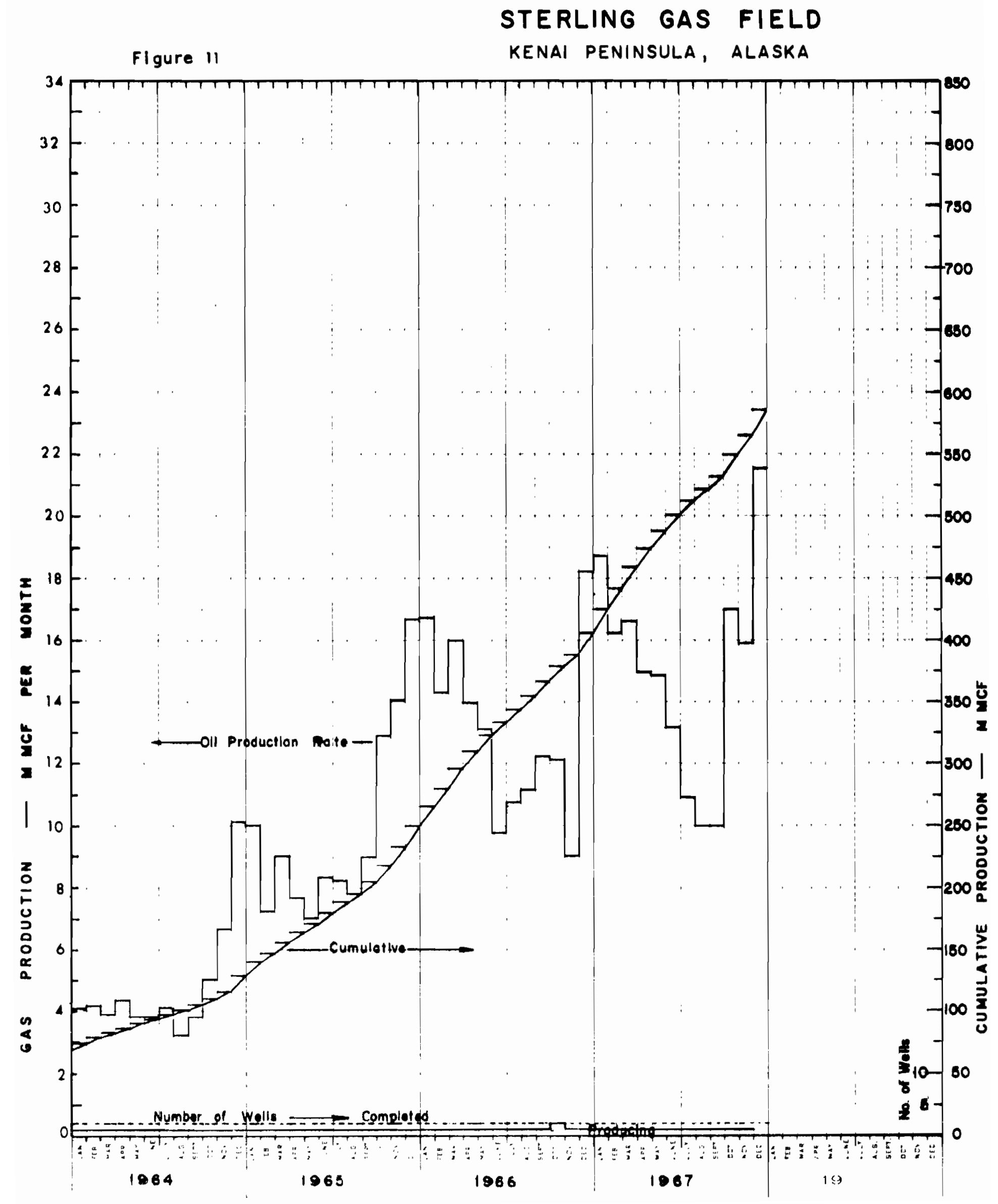




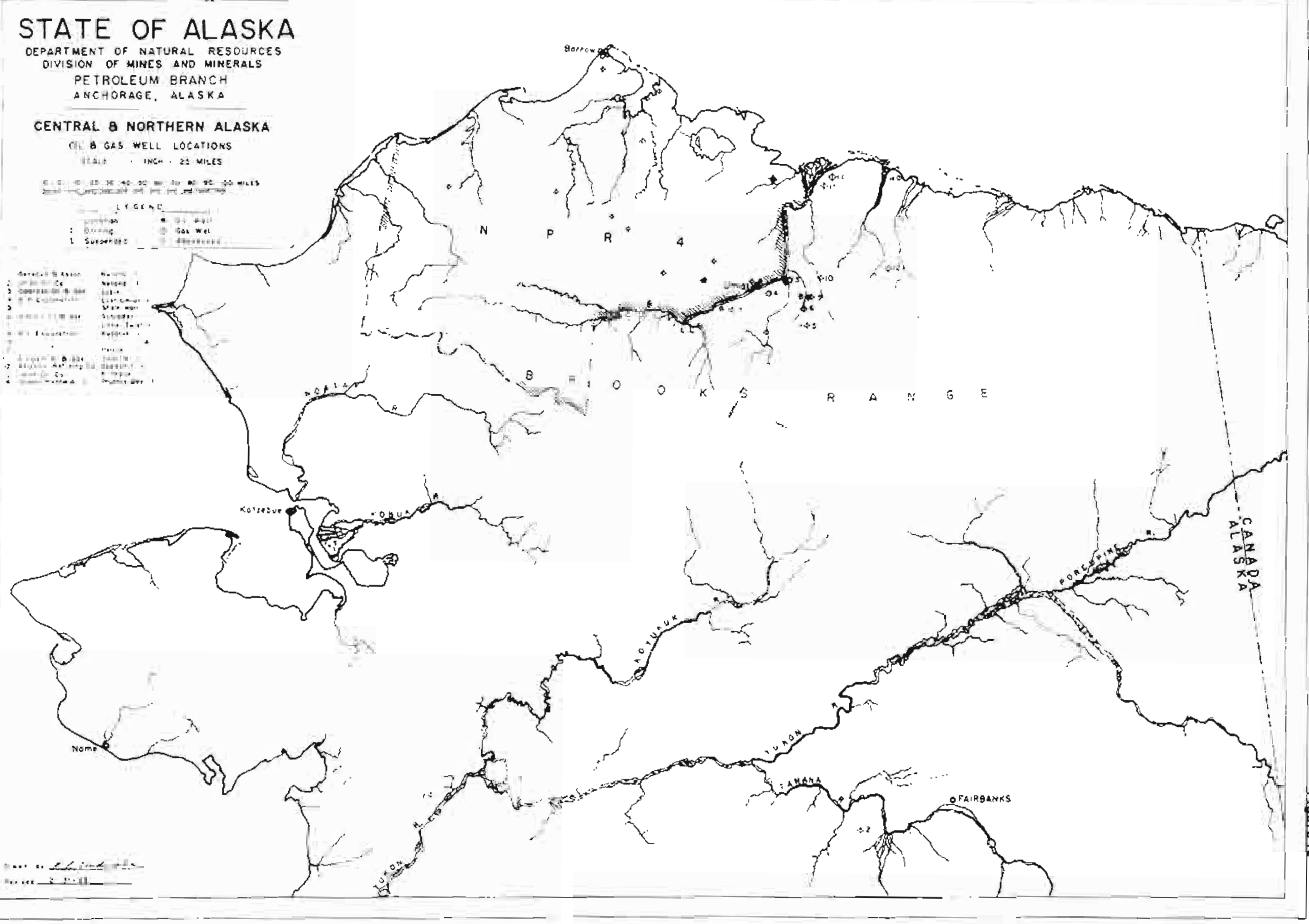




\section{ALSTRACTS OF REPORTS}

\section{FAREWELL PROGRESS REPORT}

Gordon Herreid, Mining Geologist,

Division of Mines and Minerals

The map area lies along the northern edge of the Alaska Range near Farewell in the headwaters of the Kuskokwim River. Bedrock is mainly strongly deformed, locally cross-bedded, medium gray, slate-limestone of early Paleozoic age. Also present are smaller areas of light gray massive limestone of middle (?) Paleozoic age; granitoid stocks; numerous dikes of andesite, trachyte, and rhyolite; and rhyolite flows (?). About 100 square miles were mapped and 158 stream sediment samples were taken. The area was previously unmapped.

The mapping covers part of an upland block bounded along the north edge by the Farewell fault. Recurrent movements along this great break probably have had a strong influence on the emplacement of igneous rocks in the area. A swarm of steep east-trending, mafic to acid dikes extends eastward for a distance exceeding five miles from the large granitoid stock at the head of the Middle Fork of the Kuskokwim. The swarm underlies $2 \%$ to $50 \%$ of the bedrock over a width of at least four miles. The emplacement of these dikes was probably related to movements along the Farewell fault.

Stream sediments contain scattered nickel, copper, molybdenum, and zinc anomalies in the upper Field Fork drainage. These are associated with diabase dikes and/or black slate within and marginal to the silicified aureole around the large granitoid stock at the head of the Middle Fork. One small nickel-bearing pyrrhotite deposit is associated with diabase in this area.

\section{OIL FIELDS OF COOK INLET, ALASKA}

Graydon H. Laughbaum, Jr.,

Union Oil Company of California

Significant increases in petroleum production continue in the Cook Inlet basin of Alaska, moving the state closer to being the fifth leading oil producer in the United States by 1971. Five fields account for a total present output of more than 145,000 b/d. Swanson River, Middle Ground Shoal, Granite Point, McArthur River, Foreland, Dolly Varden, and Trading Bay collectively have estimated producible reserves in excess of $750 \mathrm{million} \mathrm{bbl}$.

One of 15 geologic basins in the state, the Cook Inlet basin has an area of 9,500 sq. mi. lying approximately 45 percent offshore, 15 percent in tidal areas, and 40 percent onshore. The basin's Cenozoic stratigraphy generally is represented by a maximum thickness of $25,000+\mathrm{ft}$ of nonmarine Tertiary rocks. Mesozoic limestone, marine clastic sedimentary rocks, and voTcanic rocks unconformably underlie the Tertiary.

Major structure and fault systems trend north-northeast. Tectonism occurred throughout the Mesozoic and Cenozoic and continues into the Recent as in other areas of the circum-Pacific tectonic belt. Faulting and folding are most prominent in the northwestern part of the basin close to the Alaska Range-a range which includes dioritic and granitic mountains with numerous active volcanoes.

$0 i 1$ has been found in the classic anticlines. Production is from sandstone and conglomerate beds of the Kenai Group including the Middle Kenai and Hemlock Formations. Faulting and stratigraphic variations in part control accumulation and fluid properties. A1l reservoirs contain undersaturated oil close to bubble-point pressure requiring early secondary recovery efforts. 0 il gravities range from $25^{\circ}$ to $56^{\circ} \mathrm{API}$. Average daily production per well is about $1,400 \mathrm{bbl}$. 
NEW PETROLEUM PROSPECTS, SHALLOW AND DEEP BERING SEA
D. W. Schol1, 1
D. M. Hopkins, 1
E. C. Buffington, 2
H. G. Greene, 1

Recent geological and geophysical investigations by the United States Geological Survey and cooperating institutions have outlined three areas of possible interest for petroleum prospecting in the Bering Sea: (1) intra-shelf basins, (2) an outer-shelf sediment-draped basement high, and (3) a continental borderland, Umnak Plateau, lying seaward of the continental slope.

(1) Although the possibility of sub-shelf oil deposits has long been recognized in the thick sequence of Cenozoic sediments underlying Bristol Bay, published geophysical data seemed to indicate that elsewhere the shelf is underlain by only a thin blanket of Cenozoic sedimentary deposits overlying a basement of crystalline and deformed sedimentary rocks of Mesozoic and older age. However, our seismic reflection studies reveal that large areas of the shallow Bering Shelf are underlain by intra-shelf basins containing several thousand feet of Cenozoic deposits. For example, at least 3,000 feet of sedimentary section overlies basement in Western Norton Sound. Nunivak, St. Lawrence, and the Probilof Islands are basin-bounding structural highs; these may be flanked by oil-bearing cenozoic deposits.

(2) Reflection records reveal that the outer edge of the shallow Bering Shelf is underlain by a discontinuous basement high. The basement is composed in part of well-indurated sedimentary rocks of probable Mesozoic age. Cenozoic strata are draped over the shelf-edge basement high and bury the landward-facing flank which is thought to be the scarp of a normal fault in some areas. The high may be of some interest to petroleum geologists but possible stratigraphic and structural traps within the overlying Cenozoic section are more obvious locations for petroleum prospects.

(3) Deep-water drilling techniques will ultimately be required to explore adequately the petroleum possibilities of Umnak Plateau--the borderland which lies at a depth of 6,000 feet in the triangular area formed by the intersection of the Bering continental slope and the Aleutian Ridge. The plateau is underlain by at least 5,000 feet of Cenozoic deposits that have accumulated over a differentially downwarped portion of the basement platform underlying the shelf. The structure of the plateau is broadly domical, but moderate folding and faulting have deformed its edges; thus the flanks of the plateau may be the best location for future petroleum prospecting.

1 Office of Marine Geology and Hydrology, United States Geological Survey, Menlo Park, California 94025.

2 United States Navy Underseas Center, San Diego, California 92152.

\section{PLACER PROSPECTS IN NORTH BERING SEA}

David M. Hopkins,

U. S. Geological Survey

Possibilities of finding gold and other placer-mineral deposits in northern Bering Sea were studied in 1967 by the U.S. Geological Survey in collaboration with the University of Washington and the U.S. Bureau of Mines. The studies included seismic profiling, ship-borne magnetometry, bottom sampling, and offshore drilling.

A regional geophysical reconnaissance indicates that, contrary to expectations, bedrock is deeply buried throughout much of northern Bering Sea. Thus thick cover of sedimentary strata insulates the sea floor from most possible local sources of lode mineralization except in nearshore areas. However, glaciers have carried detrital material from onshore mineralized areas 
as far as 20 miles beyond the present shoreline. Placer concentrations of gold or tin may exist where beaches and streams have crossed these drift areas during past intervals of low sea lever.

Our studies indicate that detrital gold offshore near Nome is largely derived from mineralized bedrock and older placers onshore. Most of the gold encountered in the drilling and bottom-sampling program was found in or above glacial drift that, in the Nome area, extends only a few miles beyond the shore. A pronounced gold concentration is commoniy encountered in a thin veneer of coarse detritus covering the sea bottom; this veneer is evidently a lag concentrate of' Targe and heavy particles formed partly by present-day bottom currents and partly by wave action during the post-glacial rise in sea level.

Few drill holes encountered significant gold concentrations on bedrock. However, seismic profiles indicate the existence of fault scarps that displace the buried bedrock surface; at least one of these faults show evidence of mineralization. Future drilling should explore possible placer-gold concentrations on bedrock near the fault scarps.

PROGRESS REPORT ON THE GEOLOGY AND GEOCHEMISTRY OF THE SINUK AREA, SEWARD PENINSIJLA, ALASKA

Gordon Herreid

Mining Geologist

Division of Mines and Minerals

This report, together with Alaska Division of Mines and Minerals Report No. 24, describes the investigations made by the author in the sinuk area during 1965 and 1966. The new report consists of a geologic map of the entire area, tables of analyses, and a map and description of a new zinc-lead discovery made during 1966. This new find is located in the headwaters of Aurora Creek, a tributary to Cripple River. It shows up as a scattering of silicified, dolomitized schist and sphalerite-bearing float accompanied by a strong geochemical soil anomaly over a distance of at least 7000 feet. There are no exposures of ore in place and the deposit is largely masked by solifluction lobes.

This deposit lies five miles from the Nome-Teller highway. It is on open ground, with no mining claims in the vicinity at the time of writing. It appears to warrant enough surface work to allow an estimate of its size and grade.

\section{GEOCHEMICAL REPORT OF THE WOOD RIVER LAKES-TIKCHIK LAKES REGION}

Gilbert R. Eakins

Division of Mines and Minerals

During part of the summer of 1967 a State Division of Mines and Minerals field party collected 372 stream sediment samples in the Wood River Lakes-Tikchik Lakes region of southwest Alaska to aid in determining the mineral potential. The samples were analyzed for heavy metals by field test and by the laboratory for copper, lead, zinc, molybdenum, and mercury. The results indicate that the mercury analysis is a good geochenical tool for prospecting in the region. Zinc appeared to be the best indicator of the heavy metals group. Geochemical anomalies and other field evidence indicated the most favorable locations for exploration are: (1) Marsh Mountain, (2) the south side of the southeast end of Lake Aleknagik, (3) the ridge on the south side of Sunshine valley, and (4) the ridge on the south side of little Togiak Lake.

A GEOCHEMICAL JNVESTIGATION OF THE SOUTHWEST PORTION OF THE 40 MILE DISTRICT, EAST CENTRAL ALASKA

\section{W. H. Smith}

One hurcired and seventy-six stream sediment samples were taken in an area underlain mainly by Birch Creer. schist arid small y: whte plutons in the Eagle quadrangle, Alaska. The sample arod covers lis cquare miles and moludes the Chicken placer gold camp. Analyses of the samples for coppcr, lead, zinc, molybdenum, and nickel have indicated three areas containing moderately anomalous stream sediments near small granite plutons. 
DIVISION OF MINES AND MINERALS

General

The Division of Mines and Minerals was created to aid and promote increased mineral exploration and production in the State of Alaska. In actual practice, it is doing everything within its means to foster and encourage mineral exploratory work and new production in the best interests of the State. This helps provide the industry needed so much in Alaska.

The Division administers the laws with respect to mining and petroleum exploration and production. It is also responsible for mine safety. It operates a public assay laboratory for the purpose of stimulating discoveries and aiding bona fide prospectors and miners with free assays and mineral identifications. It does the geological mapping and other functions of a state geological survey. It provides technical advice in the field and office on prospecting and mining problems. The Division continually surveys" the mineral resources and operations in the State and disseminates this information for the assistance of prospectors, miners and petroleum operators. $0 i 1$ and gas conservation regulations are administered and enforced. By law, the Division is required to foster and promote the best interests of the mining, minerals, and related industries of the State, but it is also charged with the protection of investors in these industries. It maintains an active file or inventory of all known mineral deposits, mining claims, and mining claim owners. It provides the Division of Lands with the necessary technical help and advice on mineral leasing, unitization, discovery royalty awards, prospecting permits, and other related matters administered by that Division.

The Division of Mines and Minerals' authority and functions as delegated by the Commissioner of Natural Resources are found in the Alaska Statutes, Titles 27 and 37.

Personnel and offices of the Division formerly located in Juneau and Anchorage were moved to the campus of the University of Alaska at College (near Fairbanks) during the year. This move was directed by Governor Hickel so that the Division could better cooperate and coordinate its work with that of the University's College of Earth Sciences and Mineral Industry. Close coordination with Federal agencies to be located there in the near future is also planned. The U. S. Department of the Interior plans to establish an Arctic Mineral Resources Institute on the campus which will be staffed with scientists of the USGS and USBM. They will be housed in a building to be built for the purpose which will also house the University's CESMI and the Division of Mines and Minerals. The move of the Division to the campus was the first step in helping to implement this program. It also emphasizes the State's interest in mineral development of the North.

The Division's Petroleum Branch remains in Anchorage, the hub of petroleum activity. To continue to provide mining information in Juneau and Anchorage, a Mining Information Office was established in each of those cities staffed by a Mining Information Specialist.

Because of an unusual turnover of Personnel and interruptions caused by the move of the Division to College, field work accomplished in 1967 was much less than in the preceding two years.

Charts on the following two pages give a brief over-all view of the Division organization, personnel, functions, and a summary of work accomplished in 1967. Later pages discuss the 1967 work in greater detail and present plans for work in 1968. 
ORGANIZATION, FUNCTIONS, AND COSTS

\section{DIRECTOR}

AUIIINISTRATION

BRANCH

MINING BRANCH

3 Mining Engrs.

3 Summer Ass'ts

Secretary

clerk

3 Mining Information

Specialists

\section{College}

Juneau

Anchorage

Mineral records

information

Publishing

Budget, finance, property \& personnel

College reports

Public inquiries
METALLURGY BRANCH

\section{Authorized Personnel}

$X$-ray Minerologist

Assayer Chemist

Assayer

Minerals Lab Tech.

Clerk Typist

\section{Locations}

college

\section{Functions}

Prospect examination geochem sampling \&

Safety inspections

Technical advice \& help to prospectors and miners

Ore Assaying

geochemical, \&

other analyses

Mineral identi-

fications

Ore testing,

Research
4 Mining Geologists

4 summer Ass'ts

Clerk Typist
PETROLEUM

BRANCH

Petro. Supervisor

Petro. Geologist

4 Petro. Engineers

2 Clerk Stenos

Anchorage

College
GEOLOGY BRANCH

regulations

Technical aspects

of unitization \&

leasing regulations

Public inquiries,

Care \& release of

well logs \& samples
Ore province evaluation

Economic geology reports \& maps

ore deposit investigations

Costs (FY 67-68 Appropriations)

Division Tota] $\$ 485,600$ 


\section{SUMMARY OF WORK ACCOMPLISHED IN 1967}

\section{DIRECTOR}

Supervision and administration of Division

\section{ADMINISTRATION}

BRANCH

Accounting, budgeting, personnel, \& property administration; public information; published 10 professiona) reports, semi-annual and annual report, petroleum statistics, monthly bulletin (circulation 2200), 1504 claim location notices and 793 affidavits processed, 11,006 unpatented claims now on file, 2,000 visitors assisted, 2163 requests for information

received, 2685 professional reports sold or distributed, 1585 information circulars mailed.

\section{MINING BRANCH}

Geochemical investigations were made of three areas in which streani sediment samples were taken; 38 coal mine and several metal mine safety inspections were made, 12 prospectors were assisted under the Prospector Assistance Program. Over 400 prospectors and professional people were assisted, and over 500 letters pertaining to the mineral industry were written.

\section{METALLURGY BRANCH}

Received 2500 samples on which assays and analyses were made. Assisted a large part of the visitors.
PETROLEUM BRANCH

Approved 88 oil well drilling permits, all wells were checked for compliance with regulations, compiled production and related statistics, inventoried and made available core samples, gave technical advice to Division of Lands on leasing, royalty, and unitization matters, held five public hearings, issued nine conservation orders, drafted geologic and well drilling progress maps, witnessed well production tests.

\section{GEOLOGY BRANCH}

260 square miles of geology mapped, 110 square miles geochemical mapping, 725 stream sediment samples taken. assisted prospectors and visitors, furnished information to professional people, prepared reports and maps on previous year's investigations. 


\section{Petroleum Branch Activities}

The Petroleum Branch of the Division of Mines and Minerals issued eighty-eight drilling permits during 1967. This compares with sixty-four permits issued during 1966. Twenty-four permits were for exploratory wells and sixty-four permits for development wells. Development activity took place in four offshore oil fields and one onshore gas field.

The various reports submitted on each well were checked and filed. Production reports for each producing well in each of the five producing oil fields and two producing gas fields were checked and tabulated. Drill stem tests, production tests, and meter tests were witnessed. Technical data pertaining to discovery royalty applications were checked and recommendations were forwarded. Field trips were made to various production facilities to check safety equipment and to observe methods of waste disposal.

Seven public hearings were held during the course of the year. Testimony by Phillips Petroleum Corporation resulted in a conservation order permitting "cluster" spacing in the development of the offshore North Cook Inlet Gas Field. Conservation orders outlining field rules were written after hearings on the Middle Ground Shoal Field and Trading Bay Field. Two hearings were held on the appi ication of Pan American Petroleum Corporation for downhole commingling of various pools in one well, and for other wells in the future, in the Middle Ground Shoal Field, but a conservation order was not issued at the year's end. Also pending are the orders for Mobil 0il Corporation's application for greater well density and other field rules in the Granite Point Field and the application of Pan American Petroleum Corporation for permission to conduct fluid injection tests in the Granite Point Field. Eighteen conservation orders were issued without public hearings.

A revised and updated issue of the $0 i 1$ and Gas Conservation Regulations was distributed during 1967 to 211 who requested a copy. The regulations were revised by committees composed of representatives of 211 interested parties and the State. Public hearings on the regulations were held also.

The confidential period for samples and core chips on nineteen additional wells expired during 1967, and these are available for study at the Division of Mines and Mineral's office in Anchorage. Electric logs, lithologic logs (if required), and well reports on thirty-one wells were also made available to the public during the year.

All 0 il and gas production data for 1967 were processed and are presented in the appropriate section of this report. State, Federal, and private agencies depend on the Petroleum Branch for current production statistics. Monthly statistical reports were also prepared. Royalty and tax income forecasts were made for administrative and legislative use.

The Petroleum Branch evaluated technical data involving the outlines of proposed units and the participating areas and plans of development for approved units. 
(A summary of all Conservation Orders issued prior to 1967 is listed on page 79 of the 1966 Annual Report.)

Order No. Date Issued
Not issued

March 10, 1967

March 13, 1967

March 21, 1967

May 18, 1967

June 8, 1967

June 5, 1967

JuTy 17, 1967

JuTy 12, 1967

Ju7y 19, 1967
Pertinent Data

Application by Pan American Petroleum Corporation to commingle all production below the " $A$ " pool in the Middle Ground Shoal 17595 \#8 well was withdrawn.

Footage exception allowing the Pan American Petroleum Corporation to complete the Granite Point State 17587 \#3 we11 closer than 500 feet from a protracted section 1 ine.

Footage exception allowing the Pan American Petroleum Corporation to complete the Granite Point State 17587 \#4 well closer than 500 feet from a protracted section 1 ine.

Footage exception allowing the Union 0il Company of California to test and complete the Trading Bay \#A-3 well less than 500 feet from a section line.

Footage exception allowing the Pan American Petroleum Corporation to complete the Granite Point State 18742 \#4 well less than 500 feet from a protracted section line and less than 1000 feet from another well completed in the same pool.

In a described area of the North Cook Inlet Field, which is also the initial participating area of the North Cook Inlet Unit, the Tertiary System Gas Pool is defined and a "cluster" spacing pattern for field development is permitted; to be in force for a maximum of eighteen months.

In a described area of the Trading Bay Field, four fault block pools in the Hemlock Formation are defined, eliminated the 500 foot standback provision (except where ownership changes), retained the 1,000 foot minimum distance between wells and allowed one Hemlock well in each 80 acre tract; to be in force for a maximum of eighteen months.

Footage exception allowing the Union 0 il Company of Caljfornia to complete and produce the Trading Bay \#A-4 wel1 less than 500 feet from a protracted section 1 ine.

Footage exception allowing the Halbouty Alaska 0 il Company to drill the West Fork $\# 42-20$, a gas field development well, at a location less than 1500 feet from a section line.

In a described area at the Middle Ground Shoal Field, eliminated the 500 foot standback provision, eliminated the 1000 foot minimum distance between wells, permitted two wells per 160 acres, retained seven pools and same commingling groups, permitted fluid injection for pressure maintenance and for secondary recovery with pool restrictions, permitted a well to be drilled on the common lease line, allowed for administrative approval of routine 
JuTy 11,1967

August 3, 1967

August 3, 1967

August 3,1967

August 18, 1967

September 7, 1967

September 7, 1967

Septeniber 7, 1967

September 7, 1967

October 12,1967

October 12, 1967

Pending

Pending

Novenliber 24, 1967 changes in secondary recovery operations and established requirements for casing program, cementing, pressure surveys, and G.0.R. tests. This order supersedes Conservation orders \#26 and \#31.

Footage exception allowing the Atlantic Richfield Company to drill the exploratory well Trading Bay State \#2 at a location less than 500 feet from a protracted section line.

Footage exception allowing the Union 0il Company of California to produce the deviated development well Trading Bay \#A-5 less than 500 feet on each side of a protracted section line.

A determination that the Shell $0 i 1$ Company is already authorized by Conservation Order \#44 to produce the M.G.S. \#A-32-11 well in the same quarter section with the M.G.S. \#A-42-11.

A determination that the She $110 i 1$ Company is already authorized by Conservation Order \#44 to drill and produce the MGS A-44-11 well in the same quarter section with the MGS $A-43-11$ well.

Footage exception allowing the Mobil 0 il Corporation to produce the Granite Point State \#31-13 well less than 500 feet from a protracted quarter section 7 ine.

Footage exception allowing the Mobil 0il Corporation to produce the Granite Point State $\# 33-13$ well less than 500 feet from a protracted quarter section line.

Footage exception allowing the Tenneco 0il Company to drill the State 36465 \# well less than 500 feet from a protracted section line.

Footage exception allowing the Pan American Petroleum Corporation to complete and produce the Granite Point "A" 18742 \#7 well less than 500 feet from a protracted section line.

An order extending the special rules of Conservation Order $\# 44$ to also apply to a described area in the Southern part of the MGS field.

An order issued to correct the typograpinical error in the description of the area of Conservation order $\# 53$.

Footage exception allowing the Mobil $0 i 1$ Corporation to produce the Granite Point State $31-14$ well less than 500 feet from a protracted section line.

Footage exception allowing the Pan Allerican Petroleu:l Corporation to complete and produce the Granite point $17586:$ a Well less tilan 500 feet frum the property line. 
Table XXVIII

Well records released to the public during 1967 through local scouting services and reproduction firms in Anchorage:

\section{Operator}

Union 0 i1 Company of California B. P. Exploration Company

Humble $0 i 1$ and Refining Company B. P. Exploration Company Union $0 i 1$ Company of California Union Oil Company of California Pan American Petroleum Corporation Union 0 il Company of California Standard $0 i 1$ Company of California Union 0il Company of California Union $0 i l$ Company of California Shell 011 Company Union $0 i 1$ Company of California Union 0il Company of California Wallace Mining Company Union $0 i 1$ Company of California Pan American Petroleum Corporation Union $0 i 1$ Company of California Mobil 0 il Company She11 0il Company Union Oil Company of California Union 0il Company of California Shell 0 il Company

Pan American Petroleum Corporation Pan American Petroleum Corporation Union $0 i 1$ Company of California Pan American Petroleum Corporation Pan American Petroleum Corporation She11 0 il Company Mobil Oil Company Pan American Petroleum Corporation Shell 0 il Company
We11 Name and Number

Kenai Unit 13-8

Kuparuk \#1-A

Tyonek Reserve \#1

Itkiliik \#1

Trading Bay \#1

Kenai Unit 33-32

West Foreland Unit \#1

Kenai Unit 21-6

Birch Hi11 Unit 22-25

Kenai Unit 43-6

Kenai Unit 27-7

Middle Ground Shoal A-43-11

Trading Bay \#1-A

Kenai Unit 43-7

Wallace and Knutson \#1

Kenai Unit 11-6

Tyonek State 18742 \#1

Trading Bay \#2

Granite Point \#1

SRS State \#1

Trading Bay \#3

Grayling \#1

Middle Ground Shoal A-32-11

Tyonek State 17587 \#2

Middle Ground Shoal State 18746 \#1

Grayling \#1-A

Cook Inlet State 18740 \#1

Cook In let State 18741 \#1

Middle Ground Shoal A-23-12

Moquawkie \#1

Tyonek State 18742 \#2

SRS South Cook Inlet \#2 
Table XXIX

Well Records to be Released During 1968

With the Release Date

Operator

Standard 0 il Company of California Atlantic Richfield Company Atlantic Richfield Company She1 10 il Company

Atlantic Richfield Company

Sinclair OiT and Gas Company

Pan American Petroleum Corporation She11 0 il Company

Standard Oil Company of California Texaco, Inc.

Wallace Mining Company

Texaco, Inc.

Austral 0 il Company

Union 0il Company of California

Pan American Petroleum Corporation

Pan American Petroleum Corporation

Shell 0 il Company

Atlantic Richfield Company

She11 0il Company

Pan American Petroleum Corporation Standard 0il Company of California Union 0 il Company of California Pan American Petroleum Corporation Atlantic Richfield Company

Hunt Oi Company

Pan American Petroleum Corporation Shell 0 il Company

Great Basins Petroleum Company Trinity Canadian Drilling Company Pan American Petroleum Corporation Atlantic Richfield Company

Pan American Petroleum Corporation Shell 011 Company

Texaco, Inc.

Union Oil Company of California

Standard Oil Company of California

Atlantic Richfield Company

Texaco, Inc.

She11 0il Company

Union Oil Company of California

Atlantic Richfield Company

Pan American Petroleum Corporation

Pan American Petroleum Corporation

Pan American Petroleum Corporation

Mobil 0 il Corporation

She 11 oil Company

Pan American Petroleum Corporation

Placid 0il

Texaco, Inc.
We1 1 Name and Number

North Fork Unit \#41-35

Rainbow Federal \#1

Rainbow Federal \#2

Middle Ground Shoal \#A-32-14

Lorraine State \#1

Colville \#1

USA Edna Mae Walker \#1

Middle Ground Shoal \#A-34-14

Naptowne Unit \#24-8

Nicolai Creek \#1-A

Wallace and Knutson \#1-A

Nicolai Creek State \#1

J. M. Needham et. al. \#1

Kustatan \#1

Middle Ground Shoal State 17595 \#6

North Redoubt State 17597 \#1

Forelands Channel State \#1

Middle River State \#1

Midd1e Ground Shoal \#A-13-1

Cook Inlet State 18741 \#2

Falls Creek Unit \#2

Kustatan \#1-A

Tyonek State 17588 \#1

West Foreland Unit \#3

$01 d m a n ' s$ Bay \# 1

Middle Ground Shoal State 17595 \#7

Forelands Channel \#1-A

Ugashik \#1

Homes teaders \#1

West Foreland Unit \#2

McArthur State $\# 1$

Tyonek State 17586 \# 1

Middle Ground Shoal \#A-11-1

Trading Bay State \#1

Kenai Unit \#41-2

Ivan River \#44-1

Middle River State \#2

Nicolai Creek Unit \#2

West Foreland Unit \#4

East Trading Bay State \#1

Drift River State \# 1

North Middle Ground Shoal 18745 \#1

Cook Inlet State 17591 \#1

North Middle Ground Shoal 18745 \#1-A

Tower \#]

Middle Ground Shoal \#A-11-12

East Middle Ground Shoal 18751 \#1

State 17580 \#1

Coal Bay State \#1
Date

$1-20-68$

$1-21-68$

$2-26-68$

$3-16-68$

$3-28-68$

$4-8-68$

$5-1-68$

$5-14-68$

$5-27-68$

$5-28-68$

$6-1-68$

$6-6-68$

$6-7-68$

$7-12-68$

$7-13-68$

7-18-68

7-23-68

7-24-68

$7-27-68$

8- 6-68

8-18-68

9- 7-68

9-70-68

$9-15-68$

9-18-68

9-19-68

9-23-68

9-25-68

$10-3-68$

$10-14-68$

$10-74-68$

$10-24-68$

$10-25-68$

$10-27-68$

$10-28-68$

$11-8-68$

$11-21-68$

$11-23-68$

$12-7-68$

$12-10-68$

$12-15-68$

$12-17-68$

$12-19-68$

$12-22-68$

$12-22-68$

$12-24-68$

$12-27-68$

$12-28-68$

$12-30-68$ 


\section{SAMPLE \& CORE INVENTORY}

\section{Table $x \times x$}

We1l samples \& cores in sample storage

at 3001 Porcupine Drive, Anchorage, Alaska

*Destroyed by March 27, 1964 earthquake. Replacement is being attempted and any progress will be noted in subsequent inventories.

Samples through set No. 102 are no longer confidential.

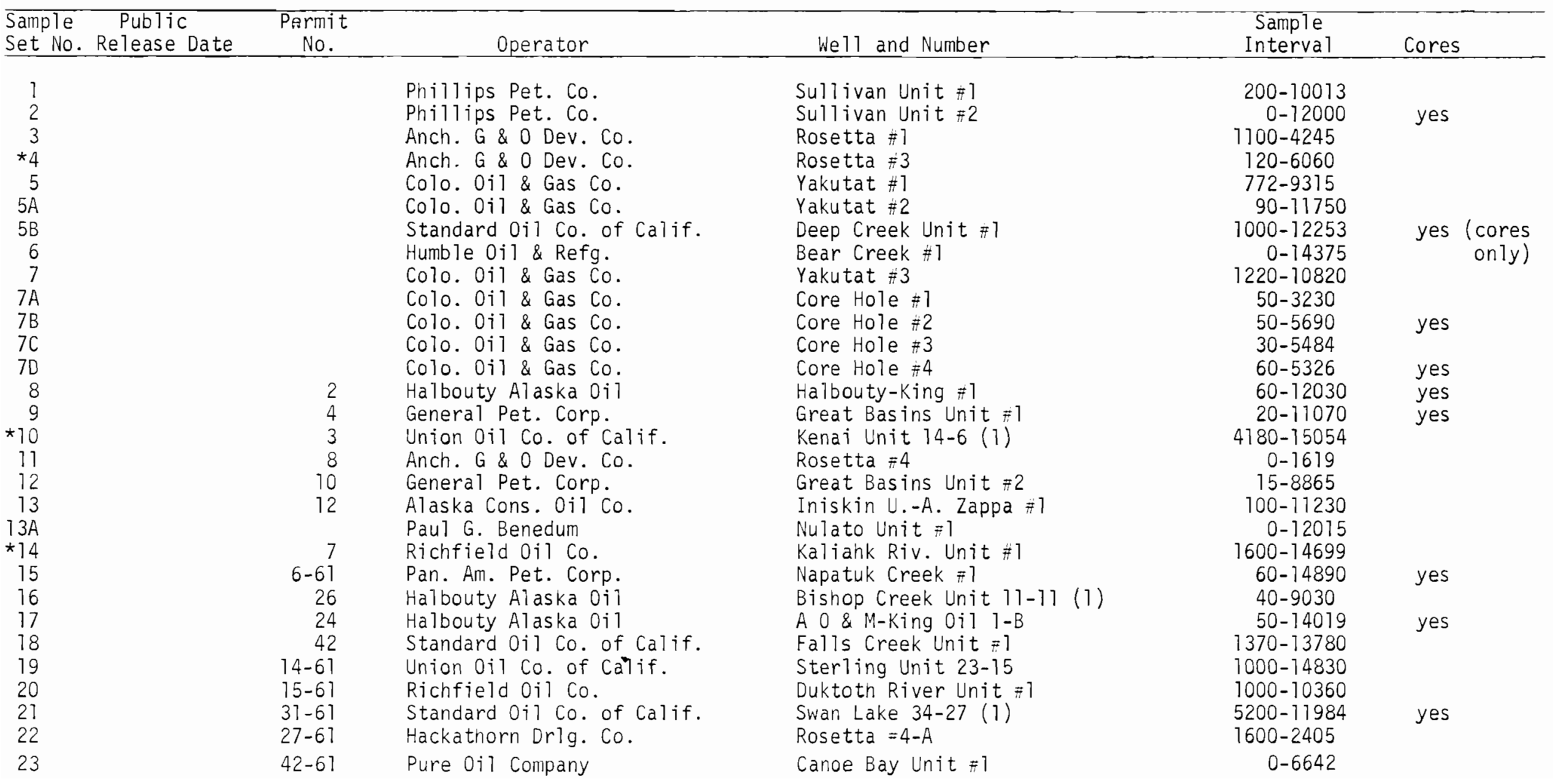




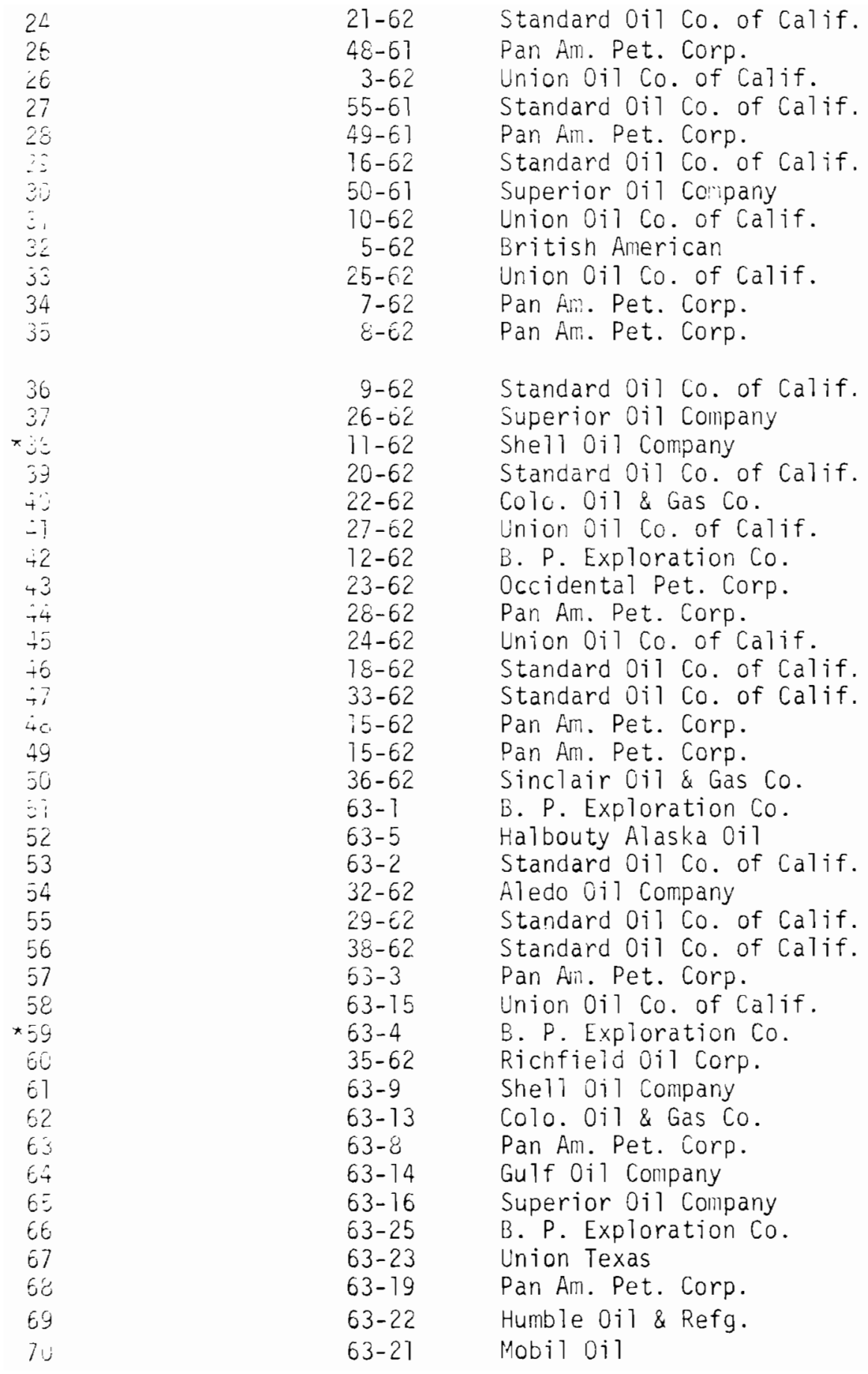

Anchor Point Unit $\neq 1$

Stedatna Creek-State $\# 1$

Nenana $\neq 1$

Soldotna Creek 34-16 (32)

West Foreland $\neq 1$

West Fork Unit 233-16

Chuit-State $=1$

Pittman Unit

Bell Island Unit $\# 1$

Sterling Unit 43-28

i. G. S. State $=1$

Cook Inlet State $\# 1$

(CI St. 17589 well No. 1)

Riou Bay Unit $=1$

Chuit State $\# 2$

SRS State 71

Soldotna Creek Unit 22-32 (34)

Malaspina Unit $\pi 1-A$

Tazlina $\neq 1$

White River Unit $\approx 2$

South Diamond Gulch \#

Cook Inlet State $* 1-A$

Ninilchik State $\#$

Beluga River Unit \#

Beluga River Unit 212-25

Tyonek State $\pi 1$

Tyonek State $\ddot{\pi} 1-A$

Swan Lake $=2$

Wasilla State $=1$

Fritz Creek

Beluga River Unit 233-27

Eureka $\approx 2$

Swanson River Unit 14-9

Soldotna Creek Unit 22A-32

Moose Creek Unit $\vec{\pi} 1$

Knik Armi State $\# 1$

White River Unit 73

Wide Bay State $\ddot{\pi}$ ?

M. G. S. State

Gubik Unit \#

M. G. S. State $\# 2$ (17595)

Sandy River Federal \#

Happy Valley Unit 31-22

Shale Wall Unit \#?

Pure-Kahiltna Riv. Unit $\#$ l

Romig Park, Inc. \#

Susitna State Unit \#l

Salmon Berry Lake \#1

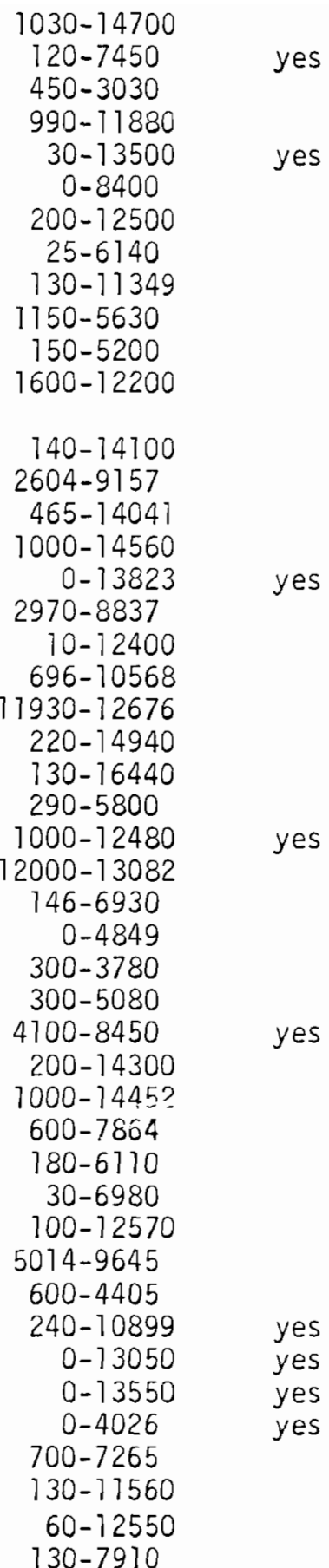




$\begin{array}{lll}71 & & 63-24 \\ 72 & & 64-4 \\ 73 & & 63-20 \\ 74 & & 54-3 \\ 75 & & 64-5 \\ 76 & & 64-8 \\ 77 & & 63-6 \\ 78 & & 64-2 \\ 79 & & 64-9 \\ 80 & & 63-18 \\ 81 & & 64-6 \\ 82 & & 64-7 \\ 83 & & 64-10 \\ 84 & & 64-14 \\ 85 & & 64-11 \\ 86 & & 64-15 \\ 87 & & 64-12 \\ 88 & & 65-1 \\ 89 & & 65-4 \\ 90 & & 65-13 \\ 91 & & 65-14 \\ 92 & & 65-18 \\ 93 & & 65-2 \\ 94 & & 11-62 \\ 95 & & 65-20 \\ 96 & & 65-19 \\ 97 & & 65-22 \\ 98 & & 65-25 \\ 99 & & \\ 100 & & 65-30 \\ 101 & & 65-23 \\ 102 & & 65-28 \\ 103 & 1-20-68 & 65-12 \\ 104 & 1-21-68 & 65-21 \\ 105 & 2-26-68 & 65-34 \\ 106 & 3-28-68 & 65-1 \\ 107 & 4-8-68 & 65-37 \\ 108 & 5-1-68 & 65-36 \\ 109 & 5-27-68 & 63-17 \\ 110 & 5-30-68 & 65-16 \\ 111 & 6-6-68 & 65-27 \\ 112 & 6-6-68 & 66-8 \\ 113 & 6-7-68 & 66-6 \\ 114 & 7-8-68 & 66-9 \\ 115 & 7-16-68 & 66-16 \\ 116 & 7-18-68 & 66-14 \\ 117 & 7-24-68 & 66-20\end{array}$

B. P. Exploration Co.

Sinclair $0 i 1$ \& Gas Co.

Standard $0 i 1$ Co. of Calif.

Mobil 0i1

Sinclair 0il \& Gas Co.

B. P. Exploration Co.

Pan Am. Pet. Corp.

Union 0il Co. of Calif.

Union Oil Co. of Calif.

Shell 0i1 Company

Pan Am. Pet. Corp.

Pan Am. Pet. Corp.

Pan Am. Pet. Corp.

Union 0il Co. of Calif.

Humble $0 i 1$ \& Refg.

B. P. Exploration Co.

Pan Am. Pet. Corp.

Standard 0il Co. of Calif.

Shell 0il Company

Union 0il Co. of Calif.

Pan Am. Pet. Corp.

Union 0il Co. of Calif.

Mobil Oil Company

Shell 0il Company

Union 0il Co. of Calif.

Pan Am. Pet. Corp.

Pan Am. Pet Corp.

Union 0il Co. of Calif.

Pan Am. Pet. Corp.

Shell 0il Company

Pan Am. Pet. Corp.

Mobil 0il Company

Standard 0 il Co. of Cal if.

Atlantic Refining Co.

Atlantic Refining Co.

Atlantic Refining Co.

Sinclair 0il \& Gas Co.

Pan Am. Pet. Corp.

Standard 0 il Co. of Calif.

Wallace Mining Co.

Texaco, Incorporated

Texaco, Incorporated

Austral 0i] Company

Union 0il Co. of Calif.

She11 0il Company

Pan Am. Pet. Corp.

Atlantic Richfield Co.
East Umiat Unit \#1

Schrader Unit \#

Beluga River Unit 14-19

Ninilchik Unit \#1

Little Twist Unit \#

Kuparuk Unit \#

Tyonek State \#2

Kasilof State \#1

Kasilof State \#2

North Cook Inlet State \#1

M. G. S. State \#4 (17595)

M. G. S. State \#3 (17595)

M. G. S. State \#6 (18743 Well \#1)

Kenai Unit 13-8

Tyonek Reserve \#1

Itkillik Unit \#1

West Foreland Unit \#1

Birch Hill Unit \#22-25

SRS-MGS State \#A-1-3 (\#A-43-11)

Trading Bay \#1-A

Tyonek State 18742 \#

Trading Bay \#2

Granite Point \#1 (State)

SRS State \#1 (drld. deeper)

Trading Bay \#3 State

Tyonek State 17587 \#2

M. G. S. State $18746 \# 1$

Grayling \#1-A

(McArthur River Field)

Cook Inlet State $18740 \# 1$

SRS S. Cook Inlet \#2

Cook Inlet State $18741 \# 1$

Moquawkie \#1

North Fork Unit \#41-35

Rainbow Federal \#1

Rainbow Federal \#2

Lorraine State \#1

Colville State \#

U. S. A. Edna Mae Walker \#1

Naptowne Unit \#24-8

Wa1lace-Knutson \#1-A

Nicolai Creek State \#

Ni colai Creek State \#1-A

Needham \#1

Kustatan \#1

Forelands Channel \#1

North Redoubt State \#1

Middle River State $\# 1$
102-3340

$0-5129$

$220-14950$

$200-12710$

$0-3600$

$0-6570$

220-12580

$1500-16121$

2050-6686

1504-14815

$180-9203$

$130-11170$

500-10710

$1160-5500$

$330-13600$

0-7750

4700-11002

$220-15500$

$320-9840$

170-6530

$376-9505$

810-6620

$110-11565$

$14040-16300$

$800-7260$

$630-12335$

260-10298

820-10227

$0-6182$

$0-15403$

$300-1740$

200-10120

246-12810

$0-3000$

$0-2793$

$0-8010$

$60-9930$

$340-16300$

$0-15226$

$0-6100$

230-8330

$3885-9300$

250-6016

$880-11852$

$0-11786$

$2170-11280$

$0-7250$ yes

jes

yes

yes

yes

yes

yes

yes

yes

yes

yes 


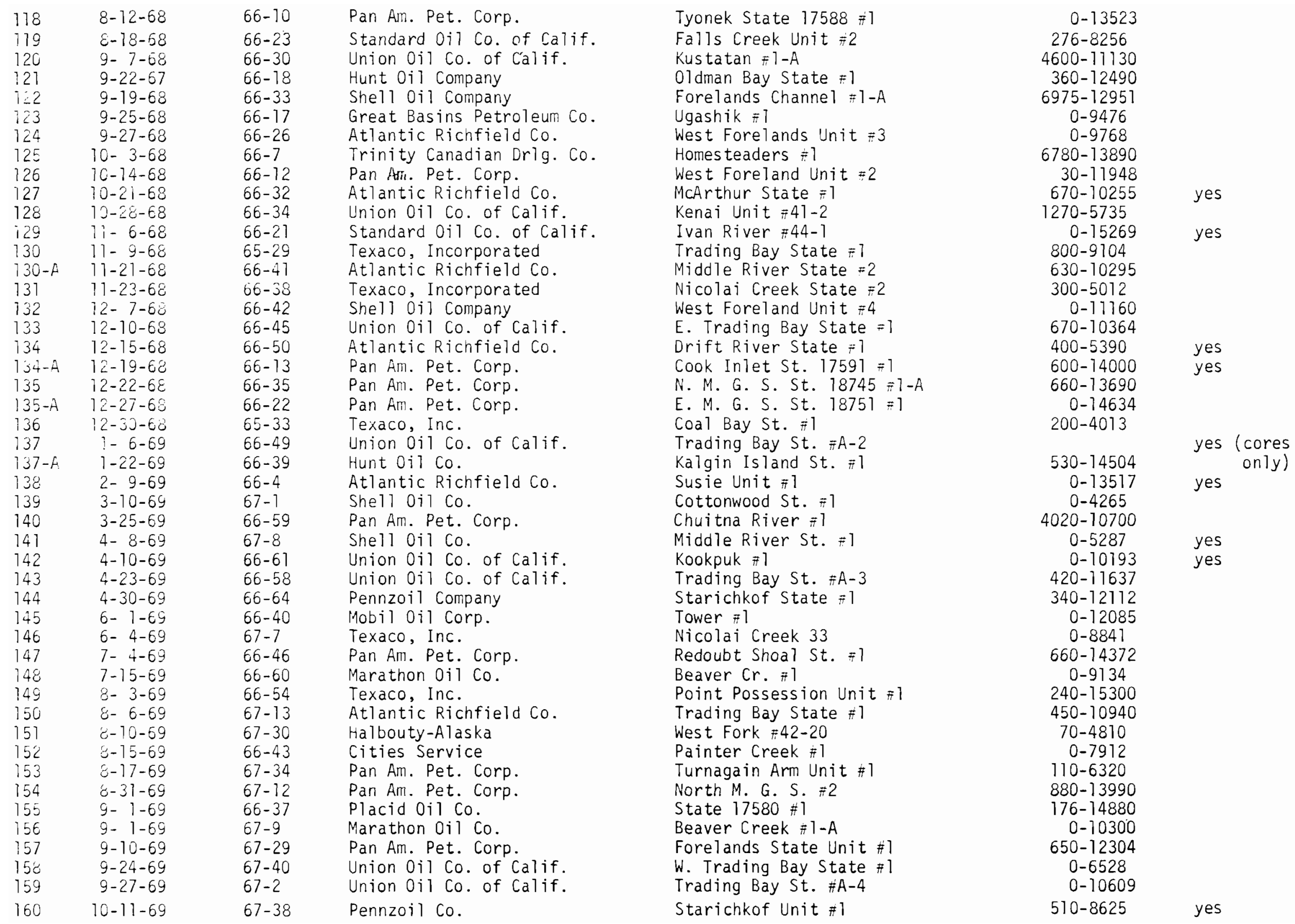




$\begin{array}{llll}161 & 10-15-69 & 67-3 & \text { Standard Oil Co. of Calif. } \\ 162 & 10-17-69 & 67-39 & \text { Shel1 0il Co. } \\ 163 & 10-24-69 & 67-37 & \text { Atlantic Richfield Co. } \\ 164 & 11-7-69 & 67-24 & \text { Mobil 0i1 Corp. } \\ 165 & 11-10-69 & 67-56 & \text { Union Oil Co. of Calif. } \\ 166 & 11-11-69 & 67-46 & \text { Union 0i1 Co. of Calif. } \\ 167 & 11-19-69 & 67-44 & \text { Atlantic Richfield Co. } \\ 168 & 11-24-69 & 67-20 & \text { Superior 0i1 Co. } \\ 169 & 12-13-69 & 67-45 & \text { Union Oil Co. of Calif. } \\ 170 & 12-15-69 & 67-43 & \text { Tennaco Oil Co. }\end{array}$
Beluga River \#14-3
Kustatan Ridge \#1
Trading Bay St. \#2
Tower \#2
Kasilof Unit \#l
Trading Bay St. \#A-7
Middle River St.
Three Mile Cr. St. \#1
Kenai Deep Unit \#
State $36465 \# 1$
$0-15196$
$30-6718$
$600-12410$
$1180-12060$
$630-5500$
$0-10586$
$30-13740$
5000-9960
2550-13963

yes (cores

yes

only)

Instructions:

At release date, most samples will be available, washed, dried, and in envelopes.

A11 sample boxes must be checked in and out of the sample room by the Petroleum Branch stenographer.

Well samples and cores may not be examined outside of the Petroleum Branch offices. All of the sample must be returned to the sample envelope. 
Reports Published During 1967

\section{Geological Reports}

No. 25. Geological and Geochemical Investigations in the Metal Creek Area, Chuadch Mountains, Alaska, by D. H. Richter. May 1967. Price \$1.00.

No. 27. Geology and Mineral Deposits of the Dolomi Arca, Prince of Wales Island, Alaska, by Gordon Herreid. June 1967. Price $\$ 1.00$

No. 28. Geology of the Upper Chistochina River Area, Mt. Haves Duadrangle, Alaska, by A. W. Rose. February 1967. Price $\$ 1.00$.

No. 30. Geology of the Upper Slana - Mentasta Pass Area, Southcentral Alaska, by U. H. Richter. May 1967. Price $\$ 1.00$.

No. 31. Geology and Stream Sediment Geochemistry of Anton Larsen Bay and Vicinitv, Kodiak Island, Alaska, by A. W. Rose and D. H. Richter. April 1967. Price \$1.00.

No. 32. Geology of an Area on the Upper Talkeetna River, Talkeetna Mountains Ouadranale, Alaska, by A. W. Rose. February 1967. Price .1 .00 .

\section{Geochemical Reports}

Ho. 3. Geochemical and Geological Investigations of Admiraltv Island, Alaska, by W. II. Race and A. W. Rose, March 1967. Price $\$ 1.00$.

No. 14. Geochemical Investigations Willow Creek Southerly to Kenai Lake Reqion Southcentral Alaska, by M. W. Jasper. June 1967. Price $\$ 1.00$.

No. 15. Geochemical Investigations Along the Valdez to Chitina Highwav in Southcentral Alaska, 1966, by M. W. Jasper, April 1967. Price \$1.n0.

Sepcial Reports:

Mineral Occurrences in the Yukon Tanana Reqion, Alaska, by R. H. Saunders. Anril 1967. Price $\$ 1.00$.

History of Mines and Prospects, Ketchikan Jistrict, Prior to 1952, bv John Bufvers. Price \$1.00. 
Reports to be Published Soon

The following reports on field work in 1967 are in prenaration, and we $n$ lan to release them prior to the 1968 field season. The listed descriptions under "area" refer only to the general areas covered and are not the exact titles that will be qiven the renorts.

\section{Geologic Reports}

No.

26

29

33
Area

Farewe11, Central Alaska Range Sinuk, Seward Peninsula Amphitheater Mountains

\section{Author \\ llerreid \\ Herreid \\ Herreid}

Geochemical Reports
Burand

Eakins

Smith 


\section{Division Programs for 1968}

Petroleum Branch

Responsibilities of the branch include drilling permits, well inspections, issuance of conservation orders, and public hearings. roilling activity is expected to maintain its present high level. As a result, the Petroleum Bri ch is still expanding. The development of each new oil and/or gas field requires the evaluati un of various data to determine correct well spacing, to delineate the pools, and to determine safe production rates. To facilitate this work steps are being taken toward the eventual machine handling of production and well records.

Geology and Mining

As in past years areas considered favorable for ore deposits of economic importance will be investigated and reports published on the results. The purpose of the program is to provide information for further field work by prospectors and mining companies.

Southeastern Alaska

Kasaan-Hetta Inlet, Prince of Wales Island: Numerous rich copper-iron deposits have been mined in this area. Geochemical, geological, and geophysical (magnetic) mapping will be conducted and prospects re-examined.

\section{Southcentral and Southwestern Alaska}

Talkeetna Mountains: Continue preliminary investigations of A. W. Rose (report No. 32) on sources of copper anomalies on the Talkeetna River and possible extension of similar occurrences in the vicinity of Iron and Sheep creeks.

Beluga and Bering River coal areas; Check exploration activities and conduct geological work where needed or advisable.

Wood River-Tikchik Lakes Region: Completion of a project started in 1967. Significant anomalies obtained last year will be investigated and mapping and sampling extended into new areas.

\section{Northcentral Alaska}

Farewell area, Kuskokwim Region: Extend detailed mapping and sampling in an area of dikes and intrusives along the Farewell fault in the Alaska Range.

Upper Koyukuk River: Examination of copper occurrences and their relation to granite intrusives for possible ore-bodies of commercial importance.

Bornite area: Detailed geological-geochemical mapping of a possible copper belt will be done along the southern flank of the Brooks Range.

Uranium investigations will be conducted along the margins of lowland basins. Northwestern Alaska

Sinuk area, Seward Peninsula: Further studies on iron deposits and lead-zinc geochemical anomalies of ore-target size.

Prospect investigations, mining district evaluations, and geochemical and geological work will be performed where advisable. 
The Division of Mines and Minerals answered 39 requests for information abnut the Prosnector Assistance Program and received 22 applications which were sent to the screeninq hoards. Two applications were denied because the applicants lacked experience in comnarison to other annlicants, and 20 applications were approved, resulting in the encumbrance of the $\$ 3 n, 000$ fund. After the fund was committed three more participants were added because thev accented the condition that reimbursement would be made if funds were available. Of the 23 narticinants, five dropped out without completing the work, 12 completed their nrograns and have been naid, and six are pending at the date of this writing.

There were 26 new claims staked by five of the prospectors, and two of the nrosnectors have made arrangements for major mining companies to investigate their firdinns. One of these investigations is developing into a major drilling project.

To date, the total cost of the 1967 program to the State of N1aska is $321,562.02$ : this amounts to $\$ 19.79$ per man-dav. Actual expenditures by the nrospectors totaled $\$ 33,163.72$ for a man-day average of $\$ 30.45$.

Members of the screening boards for the 1967 programs were:

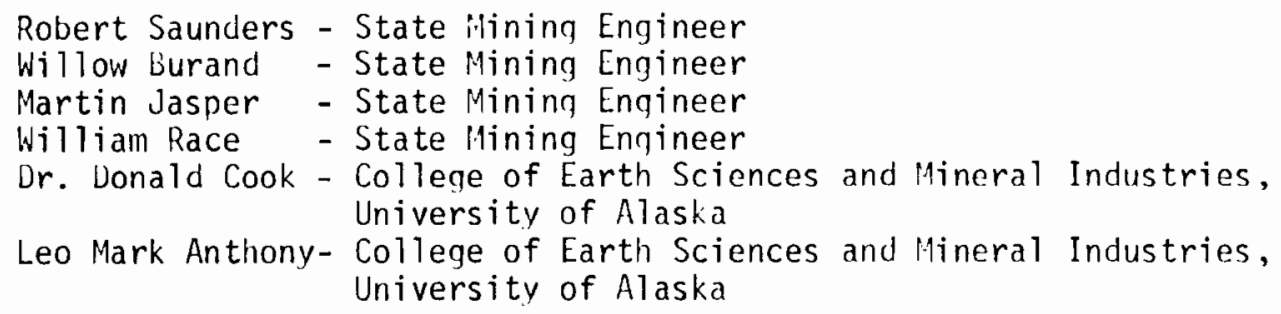

Table XXXI on the following page presents data pertinent to the 1967 Prospector Assistance Programs. 
TABLE XXXI

PROSPECTOR ASSISTANCE PROGRAM - 1967

\begin{tabular}{|c|c|c|c|c|c|c|c|c|c|c|}
\hline \multirow[t]{2}{*}{ liame } & \multirow{2}{*}{$\begin{array}{l}\text { Locality } \\
\text { Prospected }\end{array}$} & \multirow{2}{*}{$\begin{array}{l}\text { Man } \\
\text { Days }\end{array}$} & \multirow{2}{*}{ 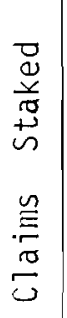 } & \multirow[b]{2}{*}{ 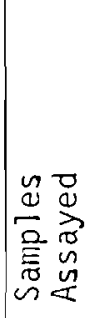 } & \multicolumn{5}{|c|}{ Actual Prospecting Expenditures } & \multirow{2}{*}{$\begin{array}{l}\text { Reimbursec } \\
\text { by State }\end{array}$} \\
\hline & & & & & Trave 1 & Food & $\begin{array}{c}\text { Equipment } \\
\& \text { Misc. }\end{array}$ & $\begin{array}{l}\text { Total } \\
\text { Expense }\end{array}$ & $\begin{array}{l}\text { Expense } \\
\text { per } \\
\text { Man-Day }\end{array}$ & \\
\hline E. Ronald Anderson & Fairbanks & 37 & 1 & 15 & 435.00 & 200.00 & 69.96 & 704.96 & 19.05 & 528.72 \\
\hline $\begin{array}{l}\text { Ed. R. Farreli \& } \\
\text { Bert tiggins }\end{array}$ & Manley Hot Springs & 82 & & 9 & 40.00 & 366.38 & $5,277.45$ & $5,633.83$ & 68.70 & $4,000.00$ \\
\hline Edwin R. Hudson & Livengood & 65 & & 5 & & 623.63 & $4,992.53$ & $5,616.16$ & 86.40 & $3,588.40$ \\
\hline $\begin{array}{l}\text { Robert F. Landel \& } \\
\text { Wm. P. Cotton }\end{array}$ & White Mountains & 56 & & & 908.28 & 377.41 & 461.24 & $1,746.93$ & 30.11 & 990.09 \\
\hline Max M. Rusaw & Matanuska & 129 & 8 & 21 & 978.30 & 248.44 & 97.39 & $1,324.13$ & 10.26 & 649.18 \\
\hline A. W. Smith & Iliamna & 54 & & & 494.50 & 510.69 & 403.65 & $1,408.84$ & 26.09 & $1,026.63$ \\
\hline Arley R. Taylor & Kantishna & 70 & 4 & & 297.22 & 379.77 & $1,903.65$ & $2,580.64$ & 36.86 & $1,037.57$ \\
\hline James A. Walper & Southeast Alaska & 225 & 4 & & $4,642.73$ & $1,125.00$ & 378.84 & $6,146.57$ & 27.32 & $4,000.00$ \\
\hline $\begin{array}{l}\text { Ernest Wolff \& } \\
\text { Lonnie Heiner }\end{array}$ & Maniey Hot Springs & 90 & 9 & & 651.00 & 406.13 & $3,824.98$ & $4,882.11$ & 54.24 & $3,619.87$ \\
\hline Eddie Chipp & Kuskokwim & 132 & & 25 & 279.65 & 394.38 & 624.29 & $1,298.32$ & 9.84 & 747.49 \\
\hline Thomas J. ivorris & Talkeetna & 67 & & & 201.98 & 102.08 & 219.14 & 523.20 & 7.81 & 392.40 \\
\hline Joe Thompson & Lake Clark & 82 & & & 540.00 & 382.18 & 376.05 & $1,298.23$ & 15.83 & 973.67 \\
\hline & TOTALS & 1089 & 26 & 75 & $9,468.66$ & $5,116.09$ & $18,579.17$ & $33,163.92$ & ! & $21,562.02$ \\
\hline & $\begin{array}{l}\text { AVERAGES } \\
\text { PER MAN-DAY }\end{array}$ & & & & 8.69 & 4.69 & 17.06 & 30.45 & & 19.79 \\
\hline
\end{tabular}




\section{ACTIVE PETROLELM COMPANIES}

Oil and gas companies active in Alaskan production, drilling, and geological and geophysical crew activities. This list does not include the many organizations involved in leasing activity only. The address given for each company is the best address at which to contact that company.

Amerada Petroleum Corp. 425 G Street, Suite 920

Anchorage, Alaska 99501

American Petrolfina lixploration Co. Room B-410 Broadway Building Denver U.S. National Center

Lenver, Colorado 80202

Apache Corporation

823 South Detroit

Tulsa, Oklahoma 74120

Ashland Oil \& Refining Co.

P. O. Box 1503

flouston, Texas 77001

Atlantic Richfield Company

P. O. Box 360

Anchorage, Alaska 99501

Austral Oil Company, Inc. 2700 llumble Building

flouston, Texas 77002

British American Oil Producing Co. P. O. Box 180

Denver, Colorado 80201

BP Exploration Co. ( $\mathrm{l}$ laska), Inc.

326 I Street

Anchorage, Alaska 99501

Cities Service Oil Company

$360 \mathrm{~K}$ Street, Suite 302

Anchorage, Alaska 99501

Clark Oil $\xi_{q}$ Refining Co.

2626 Humble Building

800 Bell Avenue

llouston, Texas 77002

Colorado Dil and Gas Corp.

P. O. Box 749

Denver, Colorado 80201

Continental Oil Company

425 G Street

Anchorage, Alaska 99501
Depco Incorporated

825 Petroleum Clul Building

Denver, Colorado 80202

Empire State Oil Company

P. O. Box 871

Thermopólis, Wyoming 82443

Great Basins Petroleum Co.

1011 Gateway West-Century City

Los Angeles, Cali formia 90067

Gulf Oil Corporation

P. O. Box 1392

Bakersfield, Califor ia 93302

llumble Oil and Refining Co.

P. O. Box 440

Anchorage, Alaska 99501

llunt Oi1 Company

326 I Street

Anchorage, Alaska 99501

Kerr-McGee Corporation

Kerr-McGee Building

Oklahona City, Oklahoma 73101

The Louisiana Land and Ixploration Co.

P. O. Box 60350

New Orleans, Louisiana 70160

Marathon Oil Company

645 G Street

Nnchorage, Alaska 99501

Mesa Petroleum Company

630 - 6th Avenue S. W.

Calgary, Alberta, Canada

Mid Continent Oil \& Gas Corp.

7857 E. Florence Avenue

Downey, Califormia 90240

Mobil Oil Company

700 G. Street

Anchorage, Alaska 99501 
Pan American I'etroleun Corp. P. 0. Box 779

Anchorage, Alaska 99501

Pennzoil Company

1007 Midland Savings Building

Midland, Texas 79704

Phillips Petroleun Corp.

P. 0. Box 419

Anchorage, Alaska 99501

Placid Oil Company

326 I Strect

Anchorage, Alaska 99501

Quintana Petroleum Corp.

19th Floor, 500 Jefferson Bldg.

louston, Texas 77002

Reserve ()il and Gas Company

550) South Flower Street

Los Nigeles, California 90017

Shell Oil Company

430 Seventh Avenue

Anchorage, Alaska 99501

Signal Oil and Gas Company

1010 Wilshire Blvd.

Los Angeles, California 90017

Sinclair Oil and Cas Company

425 (; Street

Anchorage, Alaska 99501

Skelly Oil Company

P. (). Box 1314

Anchorage, Alaska 99501

Sohio Petroleum Company

970 First National Building

Oklahoma City, Oklahoma 73102

Standard oil Co. of Cal iformia

?. O. Box 7-83?

Anchorage, Alaskal 99501

Sun Oil Company

P. O. Box 2830

ballas, Texas 75221

Sunray Iox Oil Conumany

P. (). Box 203!

Tu1sa, Oklahoma 74102
Superior Oil Company

First City National Bank Blelg.

llouston, Texas 77002

Tenneco Oil Company

P. O. Box 1703

Nichorage, Alaska 99501

Texaco, Incorporated

P. O. Box 664

Anchorage, Alaska 99501

Tidewater Oil Company

P. O. l3ox 5237

Bakersfield, California 93308

Inion Oil Company of California

2805 lhenali Street

Anchorage, Nlasha 99503 
ACTIVE COAL MINES, 1967

Name and Address of
Operator
Alaska Matanuska Coal Co.
(Paul Omlin)
Box 13, Palmer
Evan Jones Coal Co.
Box 619, Anchorage
Usibelli Coal Mines, Inc.
Usibel1i
Vitro Minerals Corp.
Box 1070, Fairbanks
Lignite Coal Sales
Lignite

Location of Mines

Type of

Operation

Approx. \& Coal Field

Strip

Crew*

Premier Mine

Matanuska Field

3

Jonesville

Strip

85

Matanuska Field

Strip

70

Healy Creek
Nenana Field

Healy Creek

Strip

41

Nenana Field

Strip

6

Lignite Creek

Nenana Field

Note: Above dald from DM\&M records.

* Size of crew is the average for the year through October. 
LIST OF ALASKA MINING \& EXPLORATION OPERATIONS

ACTIVE DURING 1967

\begin{tabular}{|c|c|c|c|}
\hline Name and Address of Operator & $\begin{array}{l}\text { Location of Mines \& } \\
\text { Recording District }\end{array}$ & Anprox. & Tvne of Oneration \\
\hline $\begin{array}{l}\text { Aho, John } \\
725 \text { 2nd Ave., Fairbanks }\end{array}$ & $\begin{array}{l}\text { Fortvmile River } \\
\text { Fairbanks }\end{array}$ & 2 & Placer nrenaration \\
\hline Agoff, S. E. & $\begin{array}{l}\text { Prince Creek } \\
\text { Mt. Mckinlev }\end{array}$ & $?$ & llonfloat \\
\hline $\begin{array}{l}\text { Ahwinona, Jacob \& Sam } \\
\text { Nome }\end{array}$ & $\begin{array}{l}\text { E. Seward Peninsula } \\
\text { Cape llome }\end{array}$ & 2 & Prosnectinn \\
\hline $\begin{array}{l}\text { Alaska Exploration \& Mining Co. } \\
\text { Talkeetna }\end{array}$ & $\begin{array}{l}\text { Bird Creek } \\
\text { Talkeetna }\end{array}$ & 1 & Hvdraulic \\
\hline $\begin{array}{l}\text { Alaska Barite Co. } \\
1112 \text { So. Cushman } \\
\text { Tacoma, Washington }\end{array}$ & $\begin{array}{l}\text { Castle Island } \\
\text { Petersburg }\end{array}$ & 20 & $\begin{array}{l}\text { Open Pit lline } \\
\text { Barite Production }\end{array}$ \\
\hline $\begin{array}{l}\text { Alaska Mines \& Minerals, Inc. } \\
\text { Box } 422 \text {, Anchorage }\end{array}$ & $\begin{array}{l}\text { Red Devil Mine } \\
\text { Kuskokwim }\end{array}$ & 1 & Develonment \\
\hline $\begin{array}{l}\text { Alaska Portland Cement Co. Ltd. } \\
136 \text { Kentucky Street } \\
\text { Petaluma, California }\end{array}$ & $\begin{array}{l}\text { Foggy Pass } \\
\text { INenana }\end{array}$ & 3 & Limestone exnloration \\
\hline $\begin{array}{l}\text { Alaska Yukon Minerals Co. } \\
i 11 \text { East } 5 \text { th Avenue, Anchorage }\end{array}$ & $\begin{array}{l}\text { Conper River Reqion } \\
\text { Chitina }\end{array}$ & 2 & Prosnectinn \\
\hline $\begin{array}{l}\text { Amax Exploration Inc. } \\
601,535 \text { Thurlow St. } \\
\text { Vancouver, E. C. }\end{array}$ & $\begin{array}{l}\text { Alaska General } \\
\text { Several }\end{array}$ & 1 & Investinations \\
\hline $\begin{array}{l}\text { American Exploration \& Mining Co. } \\
23 \text { rd Floor Russ Bidg. } \\
\text { San Francisco, California }\end{array}$ & $\begin{array}{l}\text { lluir Inlet } \\
\text { Juneau }\end{array}$ & 5 & Orilling \\
\hline $\begin{array}{l}\text { Amero, A. W. } \\
\text { Chandalar }\end{array}$ & $\begin{array}{l}\text { E. Fork Chandalar River } \\
\text { Fairbanks }\end{array}$ & 1 & Prospecting \\
\hline $\begin{array}{l}\text { Anaconda American Lrass } \\
122-744 \text { West llastings } \\
\text { Vancouver, B. C. }\end{array}$ & $\begin{array}{l}\text { Alaska reneral } \\
\text { Several }\end{array}$ & 6 & Exploration \\
\hline $\begin{array}{l}\text { Anderson, E. Roland } \\
3 / 4 \text { Mile McGrath Rd. Fairbanks }\end{array}$ & Rex Uome, Fairianks & 1 & Prosnectinn \\
\hline $\begin{array}{l}\text { Ane11, William } \\
\text { P.0. Lox 415, Rio Vell, California }\end{array}$ & $\begin{array}{l}\text { Kodiak Island } \\
\text { Kodiak }\end{array}$ & 1 & Prosnectinn \\
\hline $\begin{array}{l}\text { Asbestos Corp. Ltd. } \\
814-337 \text { W. Hastings } \\
\text { Vancouver, B. C. }\end{array}$ & $\begin{array}{l}\text { Alaska Ceneral } \\
\text { Several }\end{array}$ & 1 & [xn]oration \\
\hline $\begin{array}{l}\text { Atlantic Refining Co. } \\
\text { Box } 59 \\
\text { Ancnurage }\end{array}$ & $\begin{array}{l}\text { Alaska Peninsula } \\
\text { Iliamna }\end{array}$ & 10 & $\begin{array}{l}\text { Exnlnration } \& \\
\text { clain stakinn }\end{array}$ \\
\hline
\end{tabular}


Au Limited Henry Warner

Box 674, College

Bear Creek Hining Co.

E. 7621 Sprague

Spokane, Washington

Beckwith, Rea

Box 119, Anchorage

Berg, Rhinehart

Kobuk

Birch, Frank

Chandalar

Bliss, Patrick J. \& Sons

129 t. 11 th, Anchorage

Boedecker, Bill \& Joines, Evert Hollis

Bonanza Gold, Inc.

E. 15 Walton

Spokane, Washington

Bonne1 1, Frank

1057 W. 80th Street

Los Angeles, California

Botts, Earl \& Lyle

Box 1465, Fairbanks

Brockway, John T.

1737 Glacier Avenue

Juneau

Bronson, Robert; France, Jack \&

Wilbur

Palmer

Bunker Hill Co.

Box 29, Kellogg, Idaho

Buck, William \& Billum, Frank Glennallen

Burdick, Gordon

Busty Gelle Mine

Tury Anderson \& Associates

101 E Street, Hamilton Acres,

Fairbanks

Cambridge llining Corp. Ltd.

1130 Bay Street

Toronto, Cariada
Porcupine Creek

Fairbanks

Alaska fieneral

Several

Alaska fieneral

Several

Kugruk River

Fairhaven

Tobin Creek

Fairbanks

Ungalik Creek

Cape Nome

Hollis

Ketchikan

Prince of Wales Island Ketchikan

Kantishna District

Fairbanks

Timberline Creek

palmer

Baker Peak

Sitka

0ld Lrassel Property

Palmer

Ilannum Creek

Fairhaven

Ahtell Creek

Glennallen

iccarthy

I"cCarthy

Fairbanks

Fairbanks

Seal Cove

Ketchikan
20

Placer develonment

Exploration

Mineral investi

gations

Exploration

Ionfloat

Nonfloat

Prosnecting

Uevelopment

Lnde nrosnectinn

Strinning \& trenching

Conper develonment

fold lode mining

5

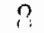

?

3

Drilling 
Canyon Creek Mining Co. Jens Kvanme \& Sons

Akiak

Carlson, Ivor C. Uphir

Carr, G. W.

lliller llouse or

4230 Altamont urive

klamath Falis, Oreqon

Casanoff, Jack

Kiana

Casto, Steve

33 Mile, llaines

Chambers, Wayne

california

Chandalar Gold Mining Co. 309 Radio Central lildr.

Spokane

Chipp, Eddie

9442 bell St. Reno, ilevada

College Road Peat

Kushman lirothers

12 Timberland ur., Fairbanks

Consolidated Wrangell llining Corn. Chitina

Cortella coal Corp.

Victor Rhine

Lox 745, Cordova

Cotton, William R.

Dart, Charles $w$.

Manley llot Springs

Davis, Bon

Lox 45, Nome

Davis Mining Co.

Bill Davis

2919 iv. 36 Street

Phoenix, Arizona

Davis linines, Inc.

Talbert $t$. Javis

1511 ilary Ann, Fairbanks

Dayo, Stanley is iveubaucr, Jack

Hanley llot Sprincs
Canvon Creek

Kuskokwill

Little Creek

$?$

IIt. IIckinlev

Circle District

Fairbanks

Klery Creek

Noatak-Kobuk

Porcupine Creek

llaines

Bluff

Cape Home

Chandalar District

Fairbanks

Farewell District

Mt. Mckinley

Colleqe Road

Fairbanks

NcCarthy

"cCarthy

Bering River Coal Field Cordova

Farewell Lake

Mt. Itckinley

Loulder Creek

Manlev llot Snrinas

Gold Run

Cane Ilome

Alaska Peninsula

Nleutian Islands

Shovel Creok

Noatak-kobuk

Cooney crepk

Manlev liot Sprinas
?

,

Ilvarautic

Nonfloat

Prosnectinn

1

Simall scale hand

Small scale hand

Dredne

Mill Construction

Prosnectinn

Peat

Surface conner

Irilling

Prosnectinn

Placer nrosnectinn

Nonfloat

Prosnectinn

exnloration

2

Nonflont 


\begin{tabular}{|c|c|c|c|}
\hline $\begin{array}{l}\text { Uickman, } 0 . \text { J. } \\
\text { Teller }\end{array}$ & $\begin{array}{l}\text { Gold Run Creek } \\
\text { Cape Nome }\end{array}$ & 4 & Hydraulic \\
\hline $\begin{array}{l}\text { Douglas, B. E. } \\
\text { Nome }\end{array}$ & $\begin{array}{l}\text { Bear Creek } \\
\text { Fairhaven }\end{array}$ & 1 & Prosnecting \\
\hline $\begin{array}{l}\text { Dynasty Exploration Ltd. } \\
330,355 \text { Burrard St. } \\
\text { Vancouver, B. C. }\end{array}$ & $\begin{array}{l}\text { IIcLean Arm } \\
\text { Ketchikan }\end{array}$ & 8 & Exploration \\
\hline $\begin{array}{l}\text { Eagle Picher Industries, Inc. } \\
\text { Box } 910 \\
\text { Miami, Oklahoma }\end{array}$ & Alaska General & 1 & Prospecting \\
\hline $\begin{array}{l}\text { Eckers, Theron } \\
\text { Kasaan }\end{array}$ & $\begin{array}{l}\text { Kasaan Peninsula } \\
\text { Ketchikan }\end{array}$ & 2 & Prosnecting \\
\hline $\begin{array}{l}\text { Edgecumbe Exploration Co. } \\
\text { C.T. \& G.H. Morgan } \\
\text { Box 758, Sitka }\end{array}$ & $\begin{array}{l}\text { Silver Bay } \\
\text { Sitka }\end{array}$ & 2 & fold lode maintanance \\
\hline $\begin{array}{l}\text { Edwards, Herk \& Miller, Vern } \\
\text { Nome }\end{array}$ & llome area & 2 & Prosnecting \\
\hline $\begin{array}{l}\text { Egnaty, Jack } \\
\text { Sleetmute }\end{array}$ & $\begin{array}{l}\text { George River } \\
\text { Kuskokwim }\end{array}$ & 1 & Exploration \\
\hline $\begin{array}{l}\text { Emerson, Fred } \\
\text { Haines }\end{array}$ & $\begin{array}{l}\text { Porcupine Creek } \\
\text { Haines }\end{array}$ & 1 & Small scale hand \\
\hline $\begin{array}{l}\text { Empire Jade Co. } \\
\text { Gene Joiner, Kotzebue }\end{array}$ & $\begin{array}{l}\text { Jade Creek } \\
\text { Noatak-Kobuk }\end{array}$ & 1 & $\begin{array}{l}\text { Jade recovery \& } \\
\text { cutting }\end{array}$ \\
\hline $\begin{array}{l}\text { Engstrom \& Son Dredging Co. } \\
\text { Box 489, Home }\end{array}$ & $\begin{array}{l}\text { Bas in Creek } \\
\text { Cape Nome }\end{array}$ & 3 & Dredqing \\
\hline $\begin{array}{l}\text { Epps, Clarence N. } \\
2440 \text { E } 20 \text { th Ave. Anchorage }\end{array}$ & $\begin{array}{l}\text { Taylor Highway } \\
\text { Fairbanks }\end{array}$ & 1 & Prospecting \\
\hline $\begin{array}{l}\text { Falconbridge Nickel Mines, Ltd. } \\
504-1112 \text { W. Pender Street. } \\
\text { Vancouver } 1, \text { B. C. }\end{array}$ & $\begin{array}{l}\text { Kasna Creek } \\
\text { Iliamna }\end{array}$ & 5 & $\begin{array}{l}\text { Geophysics \& } \\
\text { feasibility study }\end{array}$ \\
\hline $\begin{array}{l}\text { Farland, Gene } \\
\text { Box 365, Nome }\end{array}$ & $\begin{array}{l}\text { Koyana Creek } \\
\text { Cape Home }\end{array}$ & 1 & offshore nrospecting \\
\hline $\begin{array}{l}\text { Farre11, Ed } \\
5117 \text { th, Fairbanks }\end{array}$ & $\begin{array}{l}\text { Little Boulder Creek } \\
\text { Manley Hot Snrings }\end{array}$ & 2 & Drilling \\
\hline $\begin{array}{l}\text { Ferguson, Archie \& } \\
\text { Belobraidich, John } \\
\text { Kotzebue }\end{array}$ & $\begin{array}{l}\text { Candle Creek } \\
\text { Fairhaven }\end{array}$ & 6 & Nonfloat \\
\hline $\begin{array}{l}\text { Fern Gold Mining Co. } \\
502 \text { Columbia Building } \\
\text { Spokane, Washington }\end{array}$ & $\begin{array}{l}\text { Willow Creek } \\
\text { Palmer }\end{array}$ & 1 & fold lode \\
\hline $\begin{array}{l}\text { Forman, Donald } \\
\text { General Delivery, Fairbanks }\end{array}$ & $\begin{array}{l}\text { Circle District } \\
\text { Fairbanks }\end{array}$ & 1 & Prosnecting \\
\hline
\end{tabular}


Foster, Heal $W$.

Box 279, Nome

Foster, lieal W. Box 279, Ilome

Fuksa, Jim General Delivery, Anchorage

Fullerton Brothers

Gates, Willard $\mathrm{t}$.

Ghezzi, Aifred R. Box 1857, Fairbanks

Gilbertson, George

314 Charles Street, Fairbanks

Gilbertson, liarold L. Mile 1418 Alaska Highway Del ta Junction

Glass \& Heifner Jamestown, Ohio

Gold Cord Mining Co. 2309 Lard Baranof Blvd. Anchorage

Goodnews bay Mining Co. 422 White Building, Seattle, or Platinum

Greathouse, C. R., Brakefield, Erwin \& Honroe, C. , Delta Junction

Grothe, Lenhart \& Pearson, Clayton, Box 411 , Nome

Guggenheim Exploration Co. Inc. G. A. Uirom 4554 West Sixth Avenue Vancouver, B. C.

Hanria Mining Corp. 1300 Leader Building Cleveland, Ohio

liansen, burnett $F$. Eagle

Hanson, Aage 110810 th Avenue iv. Seattle, Washington

llassel Mining Co. Harold liassel Box 1071. Fairisanks
Seward Peninsula

Several

llannum Creek

Fairhaven

Kantishna

Mt. McKinlev

Little Creek

'tt. "ickinlev

Cripple Creek

it. Mckinlev

$3 r d \& 4$ th Districts

Several

Uan Creek

ilc Carthy

Richardson

Fairbanks

Beauty Bay

Seward

Fishhook Creek

Palmer

Salmon R. \& tribs.

Bethe 1

Alaska General

Several

Lost River

Cape ilome

Alaska Genera 1

Several

Alaska General

Several

Ben Creek

Fairbanks

Craigie Creek

Talkeetna

Ready Bullion Creek

Fairbanks
2
Expioration

Lode prospecting

Vionfloat

Prosnectina

Ilonfloat

Honfloat

Prosnecting

Ilonfloat

Prosnecting

Mill construction

rold lode develonment

Platinum dredqe

Prosnecting

INonfloat

Investiqations

Nonfloat

fold lode development

Nonfloat 


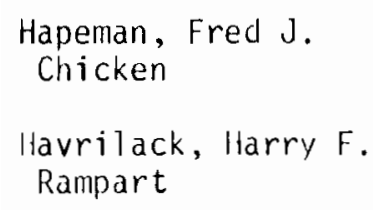

\begin{tabular}{|c|c|c|}
\hline $\begin{array}{l}\text { Buckskin Creek } \\
\text { Fairbanks }\end{array}$ & 1 & Prosnecting \\
\hline $\begin{array}{l}\text { Runnison Creek } \\
\text { Ranmart }\end{array}$ & 1 & Prosnectinn \\
\hline $\begin{array}{l}\text { Southeastern Alaska } \\
\text { Several }\end{array}$ & 1 & Prosrecting \\
\hline $\begin{array}{l}\text { Alaska feneral } \\
\text { Several }\end{array}$ & 1 & Investiqations \\
\hline $\begin{array}{l}\text { Southeastern Alaska } \\
\text { Several }\end{array}$ & 1 & Prospecting \\
\hline $\begin{array}{l}\text { Candle } \\
\text { Fairhaven }\end{array}$ & 2 & Ionfloat \\
\hline $\begin{array}{l}\text { Mud Creek } \\
\text { Fairhaven }\end{array}$ & 2 & Nonfloat \\
\hline $\begin{array}{l}\text { Grubstake Guich } \\
\text { Palmer }\end{array}$ & 1 & Soaps tone mining \\
\hline $\begin{array}{l}\text { Inmachuck River } \\
\text { Fairhaven }\end{array}$ & 1 & Hydraulic \\
\hline $\begin{array}{l}\text { American Creek } \\
\text { Manley Hot Snrinqs }\end{array}$ & 1 & llvdraulic \\
\hline $\begin{array}{l}\text { Kolmakof Property } \\
\text { Kuskokwim }\end{array}$ & 2 & "ercurv lode \\
\hline $\begin{array}{l}\text { Alaska General } \\
\text { Several }\end{array}$ & 1 & Investiqations \\
\hline $\begin{array}{l}\text { Slate Creek } \\
\text { Rampart }\end{array}$ & 1 & Ilonfloat \\
\hline $\begin{array}{l}\text { Livengood } \\
\text { Fairbanks }\end{array}$ & 2 & Ilonfloat \\
\hline $\begin{array}{l}\text { Groundhoq l3asin } \\
\text { Wranqell }\end{array}$ & 2 & Prosnecting \\
\hline $\begin{array}{l}\text { Hunter Creck } \\
\text { Pampart }\end{array}$ & ? & Ionfloat \\
\hline $\begin{array}{l}\text { Idaho Bar } \\
\text { Rampart }\end{array}$ & 1 & Ionfloat \\
\hline $\begin{array}{l}\text { Porcupine Creck } \\
\text { Haines }\end{array}$ & 1 & Small scale hand \\
\hline Alaska rieneral & $i$ & Prosnecting \\
\hline
\end{tabular}




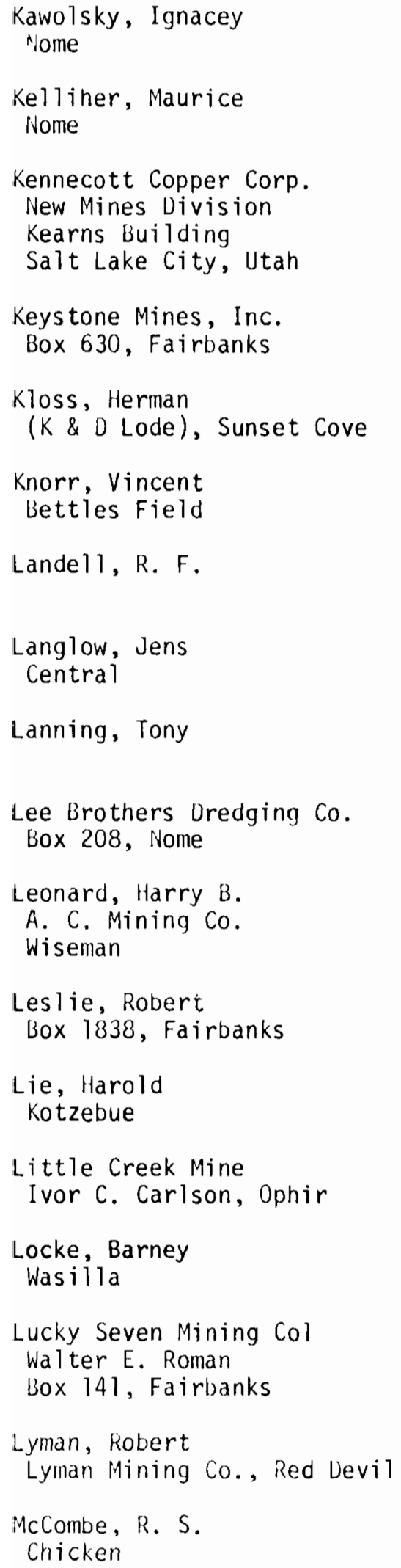

$\begin{array}{lcc}\begin{array}{l}\text { Charley Creek } \\ \text { Cape Nome }\end{array} & 1 & \text { Prosnecting } \\ \begin{array}{l}\text { Kougarok River } \\ \text { Cape Nome }\end{array} & 1 & \text { Prospecting } \\ \begin{array}{l}\text { Ruby Creek } \\ \text { Noatak-Kobuk }\end{array} & 75 & \begin{array}{l}\text { Copper lode } \\ \text { development }\end{array}\end{array}$

Wolf Creek

Fairbanks

Sunset Cove

Juneau

Mascot Creek

Koyukuk

Farewell Lake

Mt. Mckinley

Switch Creek

Fairbanks

Thanksqiving Creek

Manley Hot Springs

\begin{tabular}{|c|c|c|}
\hline $\begin{array}{l}\text { Cape Creek } \\
\text { Cape Nome }\end{array}$ & 5 & Tin development \\
\hline $\begin{array}{l}\text { Vermont Creek } \\
\text { Fairuanks }\end{array}$ & 1 & Small scale hand \\
\hline Manley Hot Springs & 2 & Prosnecting \\
\hline $\begin{array}{l}\text { Bear Creek } \\
\text { Cape Nome }\end{array}$ & 1 & Prospecting \\
\hline $\begin{array}{l}\text { Ophir } \\
\text { Mt. Mckinley }\end{array}$ & 2 & Nonfloat \\
\hline $\begin{array}{l}\text { Sheep Mt. \& Soda Creek } \\
\text { Anchorage \& Nebesna Quads }\end{array}$ & 1 & Conper prospects \\
\hline $\begin{array}{l}\text { Fish Creek } \\
\text { Fairbanks }\end{array}$ & 3 & Nonfloat \\
\hline $\begin{array}{l}\text { White Mountain } \\
\text { Kuskokwim }\end{array}$ & 4 & Cinnabar production \\
\hline Lost Chicken Creek & 1 & Prosnectinn \\
\hline
\end{tabular}

Gold lode minina

Prospecting

Nonfloat

Prospecting

Hydraul ic

1 Nonfloat

Fairbanks 


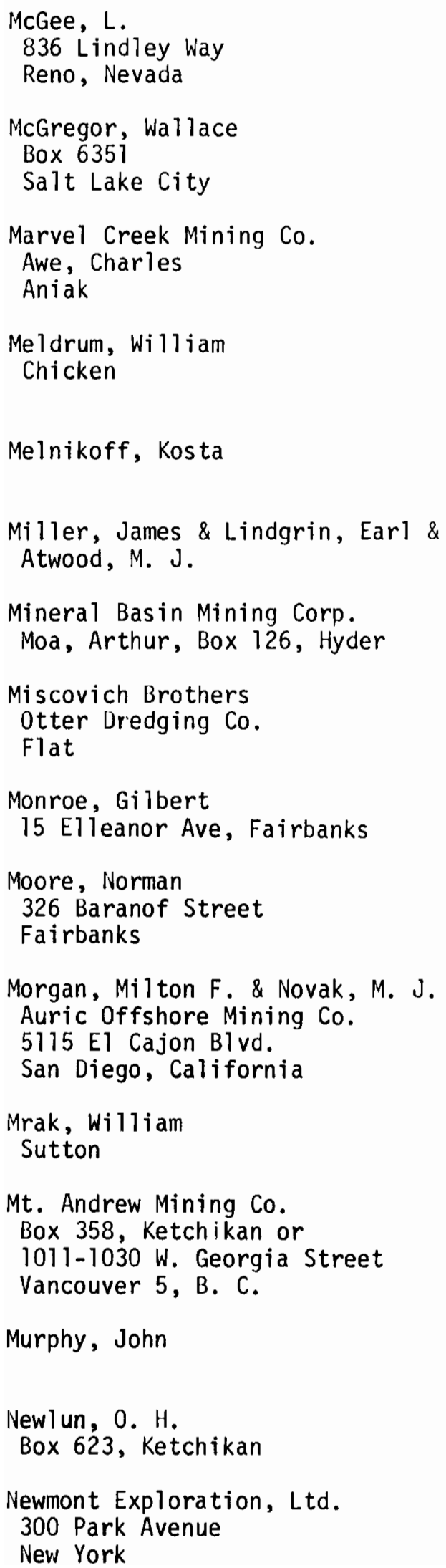

\begin{tabular}{|c|c|c|}
\hline $\begin{array}{l}\text { Cache Creek } \\
\text { Manley Hot Springs }\end{array}$ & 5 & $\begin{array}{l}\text { Development } \\
\text { Nonfloat }\end{array}$ \\
\hline $\begin{array}{l}\text { Alaska General } \\
\text { Several }\end{array}$ & 7 & Exploration \\
\hline $\begin{array}{l}\text { Marvel Creek } \\
\text { Bethel }\end{array}$ & 4 & Dredge \\
\hline $\begin{array}{l}\text { Stonehouse \& Chicken } \\
\text { Creeks } \\
\text { Fairbanks }\end{array}$ & 1 & Stripping \\
\hline $\begin{array}{l}\text { Idaho Bar } \\
\text { Rampart }\end{array}$ & 2 & Nonfloat \\
\hline $\begin{array}{l}\text { Koyukuk District } \\
\text { Fairbanks }\end{array}$ & 3 & Prospecting \\
\hline $\begin{array}{l}\text { Mt. View Property } \\
\text { Ketchikan }\end{array}$ & 2 & Exnloration (lode) \\
\hline $\begin{array}{l}\text { Otter Creek } \\
\text { Mt. McKinley }\end{array}$ & 4 & Dredge \\
\hline $\begin{array}{l}\text { Boulder Creek } \\
\text { Manley Hot Springs }\end{array}$ & 2 & Placer develonment \\
\hline $\begin{array}{l}\text { W. Fork Chistochina River } \\
\text { Chitina }\end{array}$ & 4 & Prospecting \\
\hline $\begin{array}{l}\text { Bluff } \\
\text { Cape Nome }\end{array}$ & 4 & Prospecting offshore \\
\hline $\begin{array}{l}\text { Grubstake Gulch } \\
\text { Palmer }\end{array}$ & 3 & Nonfloat \\
\hline $\begin{array}{l}\text { Kasaan Peninsula } \\
\text { Ketchikan }\end{array}$ & 1 & $\begin{array}{l}\text { Iron \& copper } \\
\text { exploration }\end{array}$ \\
\hline $\begin{array}{l}\text { George River } \\
\text { Kuskokwim }\end{array}$ & 1 & Prosnecting \\
\hline $\begin{array}{l}\text { Prince of Wales Island } \\
\text { Ketchikan }\end{array}$ & 1 & Prospecting \\
\hline $\begin{array}{l}\text { Alaska General } \\
\text { Several }\end{array}$ & 10 & Exploration \\
\hline
\end{tabular}

Cache Creek

Alaska Genera 1

Marvel Creek

Bethel

tonehouse \& Chicken

Creeks

Idaho Bar

Rampart

Koyukuk District

perty

Otter Creek

Mt. Mckinley

Boulder Creek

W. Fork Chistochina River 4 Chitina

Bluff

Cape Nome 
Norris, Thomas $\mathrm{J}$. 908 W. 57th Anchorage

North American Dredge Co. John Stevens, Flat

Northl and Mines Charles W. Monroe Box 876, Delta Junction

Novak, John 1780 0cean Blvd.

Coos Bay, Oregon

0 live Creek Mines

Carl Parker

Box 552, Fairbanks

01son, Henry T. "Tiger"

Taku Harbor

O'Neill Ventures

William O'Neill

505 8th Ave., Anchorage

Pade, Otto

Skagway

Palmer, R. B.

Box 1617, Fairbanks

Pan American Petroleum Corp.

P. 0. Box 591

Tulsa, Oklahoma

Pannick, Harry

Generai Delivery, Fairbanks

Parent, Vern \& Sorlee, Oswald Anchorage

Parker, Fred

Pederson, Steve

Box 685, Nome

Permanente Cement Co.

Oakland, California

Phelps Dodge Corp. of Canada Ltd. 404-11112 West Pender St.,

vancouver $1, B$. C.

Pieper, Paut

Box 1294, Ketchikan

Pittman, Ray

1701 Stanton Avenue

Anchorage
Nowitna

Mt. McKinley

Flat Creek

Mt. McKinley

Alaska Range

Several

6

Bering Sea \& 0thers

2

Cape Nome

Amy Creek

Fairbanks

Juneau \& Admiralty

Districts

Dan Creek

McCarthy

Skagway

Skagway

Sourdough Creek

Fairbanks

Alaska Peninsula

Iliamna

Flume Creek

Fairbanks

McKinley Creek

Haines

Inmachuck River

Fairhaven

Niukluk River

Cape Nome

Kings River

Palmer

Alaska General

Several

Kasaan

Ketchikan

A. ameda Creek

Manley Hot Springs

1

3

10

2

2
Dredque

Prosnecting

Offshore prospecting

Nonfloat

Prosnecting

Development work

Prospecting

Prospecting

Exploration

Nonfloat

Hydraulic

Nonfloat

Dredae

Limestone exploration

Investigations

Prosnecting

Nonfloat 
Springer, Karl

502-1200 West Pender Street

Vancouver $1, B$. C.

Spruce Creek Mining Co. 607 Clara Street, Fairbanks

Standard Metals Corp.

Box 1081, Ketchikan

Stanley, Kirk

7003 Mink Place

Anchorage

Steers, $A 1$

Box 826, Ketchikan

Stelting, H. W.

Box 19, Haines

Stevens, John

Strandberg Mines, Inc.

Box 2099, Anchorage

Stuver, Jules

Flat

$T$ and $T$ Mining Co.

William Thomas

Box 1464, Fairbanks, or

Rampart

Taylor, Arley \& Associates 2916 - 236th S. W.

Alderwood Manor, Washington

Tennessee Corporation

61 Broadway, New York

Thorgard, 01e

Titus, Jack \& Cook, Fred

Solomon

Totem Expioration Co.

Joe Blazek

317 Dock St., Ketchikan

Tweet, N. B. \& Sons

Teller

Uotila, Gus

Ophir

U.S.S.R. \& M. Co. Box 438, Nome
White River

Fairbanks

Spruce Creek

Mt. McKinley

Kendrick Bay

Ketchikan

Slana District

Chitina

Southeastern Alaska

Several

Haines

Haines

Flat Creek

Mt. McKinley

Tofty

Manley Hot Springs

Moore Creek

Mt. McKinley

Hunter Creek

Rampart

Kontishna District

Fairbanks

Pass Creek

Talkeetna

Kuskulana River

McCarthy

Shovel Creek

Cape Nome

Southeastern Alaska

Several

Kougarok River

Cape Nome

Birch Creek

Nulato

Northern Alaska

Several
2

Nonfloat

Prospecting

Nonfloat

Development

Drilling

Prospecting

Prospecting

Dredge

Nonfloat

Hydraulic

Nonfloat

Drilling copper

2

Prospecting

2

Small scale hand

Prospecting

6

Nonfloat

Stripping 


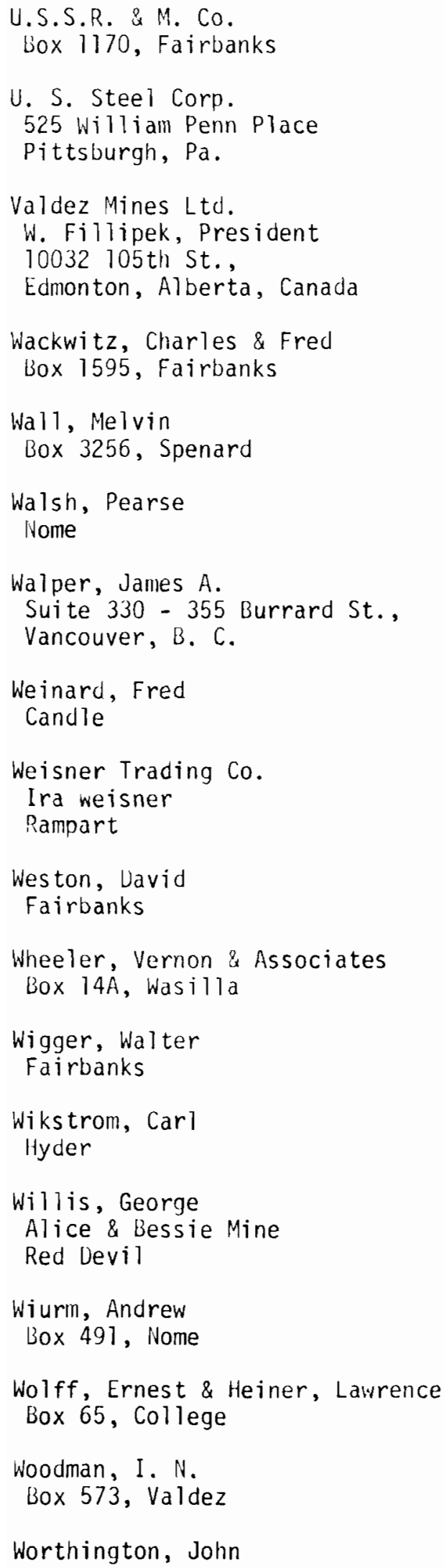

llosquito Fork

Fairbanks

Alaska General

Several

Canyon Creek

Chitina

Bedrock Creek

Fairbanks

Valdez Creek

palmer

Mt. Distin

Cape. Nome

Southeastern Alaska

Ketchikan

Mud Creek

Fairhaven

Little Minook \& Hoosier Creeks

Rampart

Dome Creek

Fairbanks

Grubstake Gulch

Palmer

Eva Creek

Fairbanks

Kantishna

Mt. Mckinley

Parks Property

Kuskokwim

Dome Creek

Cape Nome

Boulder creek

Manley llot Springs

Tonsina Lake area

Valdez

Prince of Wales Island Ketchikan
2

15

Dredae

8

Development

llickel-copner orosnecting

Prosnecting

Placer develonment

Prosnecting

Prospecting

Nonfloat

Development

Nonfloat

rold lode develonment

Ilonfloat

Prosnectinn

Small mercury oneration

Hvdraulic

Placer prosnectinn

Prospecting

Prosnecting 
*Report of the Commissioner of Mines, biennium ended December 31, 1958.

*Report of the Division of Mines and Minerals for the year 1959.

Report of the Division of Mines and Minerals for the year 1960.

*Report of the Division of Mines and Minerals for the year 1961.

*Report of the Division of Mines and Minerals for the year 1962.

Report of the Division of Mines and Minerals for the year 1963.

Report of the Division of Mines and Minerals for the year 1964.

Report of the Division of Mines and Minerals for the year 1965.

*Joesting, Henry R., Strategic Mineral Occurrences in Interior Alaska, Pamphlet No. 1 , May 1942.

*Joesting, Henry R., Supplemental to Pamphlet No. 1 - Strategic Mineral Occurrences in Interior Alaska; Pamphlet No. 2, March 1943.

*Anderson, Eskil, Mineral Occurrences other than Gold Deposits in Northwestern Alaska: Pamphlet No. 5-R, May 1944.

*Stewart, R. L., Prospecting in Alaska (26-page pamphlet) December 1944. (Revised to November 1949).

*Glover, A. E., Industrial Minerals as a Field for Prospecting in Alaska, including a Glossary of Elements and Minerals (82-page booklet) March 1945. (Revised to May 1946).

*Anderson, Eskil, Asbestos and Jade Occurrences in the Kobuk River Region, Alaska:

Pamphlet No. 3-R, May 1945.

*Roehm, J. C., Some High Calcium Limestone Deposits in Southeastern Alaska: Pamphlet No. 6, March 1946. Mimeographed copies are available.

Information Circular \#1: Proper Claim Staking in Alaska; Revised April 12, 1966.

Information Circular \#2: Mineral Rights of Aliens in the State; Revised October 28, 1966.

Information Circular \#3: Hand Placer Mining Methods, April 16, 1962.

Information Circular \#4: Uranium Prospecting in Alaska; Revised December 12, 1966.

Information Circular \#5: General Alaskan Mineral Information; Revised March 14, 1966.

Information Circular \#6: Alaskan Prospecting Information; Revised December 13, 1966.

*Information Circular \#7: Compulsory Assessment Work Affidavits, July 15, 1957.

Information Circular \#8: Mineral Industry Consultants Available for Work in Alaska; Revised December 16, 1966.

Information Circular \#9: Dealers in Alaskan Rocks and Minerals; Revised July 23, 1965.

Information Circular \#10: Skin Diving for Gold in Alaska, November 5, 1964.

Information Circular \#11: List of Division of Mines and Minerals publications; Revised December 16, 1966. 
Information Circular \#12: Services of the Division of Mines and Minerals; Revised November 9, 1966.

Information Circular \#13: Dangers in 01d Mine Openings, November 6, 1962.

Information Circular \#14: Mining Laws Applicable in Alaska; Revised November 10, 1966.

Race, William H., The Mineral Industry of the Kenai-Cook Inlet-Susitna Regions, 1962.

*Report No. PE 85-22; Report on Preliminary Investigation of the Kings River Area Limestone Deposits, Anchorage Quadrangle, by Martin W. Jasper and Miro Mihelich, State Mining Engineers, January 1961.

Report No. PE 65-1; Report on the Mespe]t Mine of Strandberg Mines, Inc., Nixon Fork District Medfra Quadrangle, Alaska, by Martin W. Jasper, State Mining Engineer, February 1961.

Alaska's New Mining Law for State Lands, by James A. Williams, Director, State Division of Mines and Minerals, December 1961 (Reprinted from Mining Engineering Magazine).

Geology and Ore Deposits of Alaska, by Gordon Herreid, Geologist, State Division of Mines and Minera1s, December 1961 (Reprinted from Mining Engineering Magazine).

Tectonics and Ore Deposits in Alaska, by Gordon Herreid, Mining Geologist, State Division of Mines and Minerals. Presented at the 1964 Alaska AIME conference, College, Alaska, March 19, 1964.

A Possible Guide to Metal Deposits of Alaska, by Charles F. Herbert, Deputy Commissioner, State Department of Natural Resources. Presented at the 1964 Alaska AIME conference, College, Alaska, March 20, 1964.

Map: Better-Known Mineral Deposits, Possible Petroleum Provinces, and Existing Roads.

Map: M.I. Report 194-1; A Preliminary Map of the Bedrock Geology of the Fairbanks Mining District, Alaska, by Robert B. Forbes and Jim M. Brown, Department of Geology, College of Earth Sciences and Mineral Industry, University of Alaska for the Division of Mines and Minera1s, December 1961. Price: \$1.00.

Geologic Report \#1: Preliminary Report on Geologic Mapping in the Coast Range Mineral Belt, by Gordon Herreid. This report formerly included in Annual Report of the Division of Mines and Minerals for the year 1962 but supply exhausted. Now available in reprint form. Price \$1.00.

*Geologic Report \#2: Bedrock Geology of the Rainbow Mountain Area, Alaska Range, Alaska; an M.S. thesis prepared by Larry G. Hanson of the University of Alaska in cooperation with the Division of Mines and Minerals. November 1963.

Geologic Report \#3: Geology of the Portage Creek-Susitna River Area, by Donald Richter, 1963. (2 large sheets). Price $\$ 1.00$.

Geologic Report \#4: Geology and Mineral Deposits of the Denali-Maclaren River Area, Alaska, by M. A. Kaufman, May 1964. (19 pages \& large map). Price $\$ 1.00$.

Geologic Report \#5: Geology of the Niblack Anchorage Area, Southeastern Alaska, by Gordon Herreid. May 1964. (10 pages \& large map). Price $\$ 1.00$.

*Geologic Report \#6: Geology and Mineral Deposits of the Ahtell Creek Area, Slana District, Southcentral Alaska, by Donald H. Richter, May 1964. (17 pages \& large map). Price \$1.00.

Geologic Report \#7: Geology of the Dry Pass Area, Southeastern Alaska by Gordon Herreid and M. A. Kaufman, June 1964. (16 pages). Price $\$ 1.00$. 
Special Reports:

Mineral Occurrences in the Yukon Tanana Region, Alaska, by R. H. Saunders. April 1967. Price $\$ 1.00$.

History of Mines and Prospects, Ketchikan District, Prior to 1952, by John Bufvers. Price $\$ 1.00$.

* Out of Print. On file in certain public and University libraries. 